Portland State University

PDXScholar

7-23-1993

\title{
Unified Bias Analysis of Subspace-Based DOA Estimation Algorithms
}

Yang Lu

Portland State University

Follow this and additional works at: https://pdxscholar.library.pdx.edu/open_access_etds

Part of the Electrical and Electronics Commons

Let us know how access to this document benefits you.

\section{Recommended Citation}

Lu, Yang, "Unified Bias Analysis of Subspace-Based DOA Estimation Algorithms" (1993). Dissertations and Theses. Paper 4613.

https://doi.org/10.15760/etd.6497

This Thesis is brought to you for free and open access. It has been accepted for inclusion in Dissertations and Theses by an authorized administrator of PDXScholar. Please contact us if we can make this document more accessible: pdxscholar@pdx.edu. 
AN ABSTRACT OF THE THESIS OF Yang Lu for the Master of Science in Electrical Engineering presented July 23, 1993.

Title: Unified Bias Analysis of Subspace-Based DOA Estimation Algorithms.

\section{APPROVED BY THE MEMBERS OF THE THESIS COMMITTEE:}

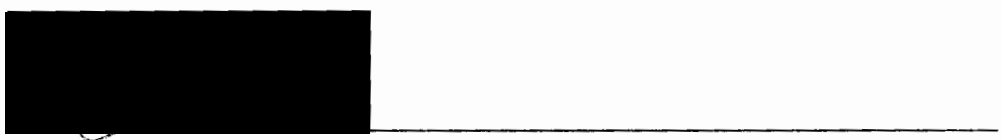

Fu Li, Chair

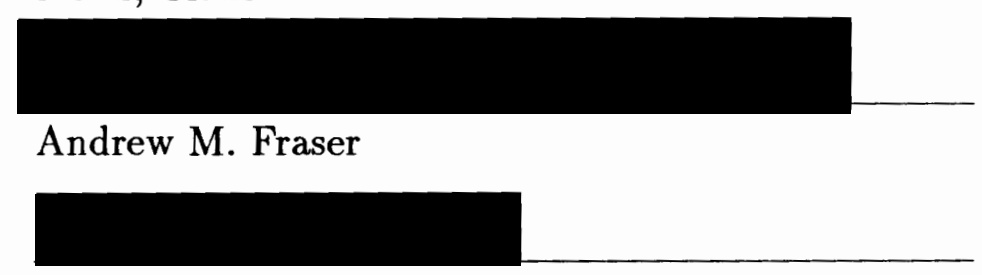

Kwok-Wai Tam

This thesis presents the unified bias analysis of subspace-based DOA estimation algorithms in terms of physical parameters such as source separation, signal coherence, number of senors and snapshots. The analysis reveals the direct relationship between the performance of the DOA algorithms and signal measurement conditions. Insights into different algorithms are provided.

Based upon previous first-order subspace perturbations, second-order subspace perturbations are developed which provide basis for bias analysis and unification. Simulations verifying the theoretical bias analysis are prusented. 
UNIFIED BIAS ANALYSIS OF SUBSPACE-BASED DOA ESTIMATION ALGORITHMS

\author{
by \\ YANG LU
}

A thesis submitted in partial fulfillment of the requirements for the degree of

\author{
MASTER OF SCIENCE \\ in \\ ELECTRICAL ENGINEERING
}

Portland State University

1993 
TO THE OFFICE OF GRADUATE STUDIES:

The members of the committee approve the thesis of Yang Lu presented July $23,1993$.

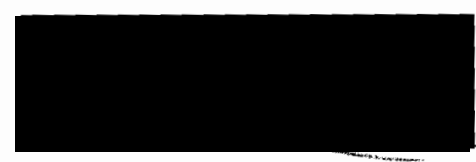

$\mathrm{Fu} \mathrm{Li}$, Chair

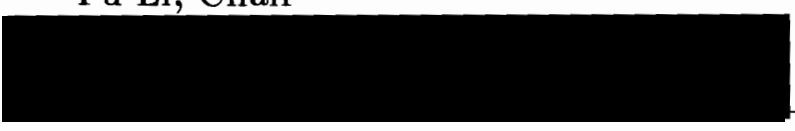

Andrew M. Fraser

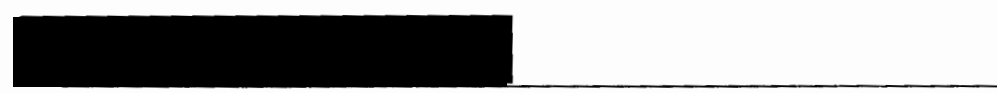

Kwok-Wai Tam

APPROVED:

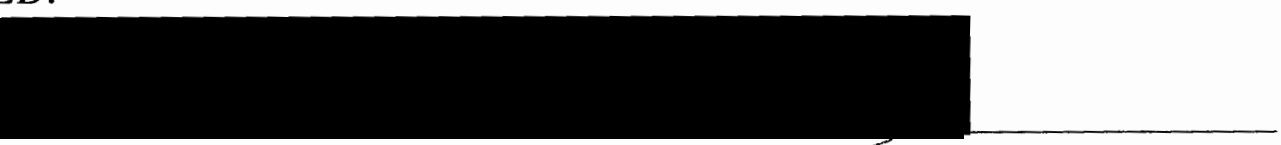

Rolf Schaumann, Chairman, Department of Electrical Engineering Roy W. Koch, Vige Provost for Graduate Stud

Roy W. Koch, Vife Provost for Graduate Studies and Research 


\section{ACKNOWLEDGEMENT}

I would like to express my gratitude to my advisor, Dr. $\mathrm{Fu} \mathrm{Li}$, for his invaluable guidance, encouragement and unrelenting patience during the course of this research.

My sincere appreciation goes to the other members of the committee, Dr. Andrew M. Fraser and Dr. Kwok-Wai Tam for their helpful comments and understanding. I wish to thank other faculty members and staffs in Portland State University who have contributed greatly to my graduate education.

Finally, my parents, deserve my special thanks for their continued support, encouragement and sacrifice thronghout, and I will forever be indebted to them for all that they have done. 


\section{PREFACE}

This work started in early 1992 , which continued the research in performance analysis of subspace based DOA estimation algorithms. The goal is to study the bias performance as co mpared to the MSE (mean square error) analysis which has been developed successfully in early 1991. Under the guidance of Dr. Fu Li, we first studied the bias performance of ESPRIT [1].(The paper has been accepted by IEEE transction on Antennas and Propagation.) Then we extended our research to include MUSIC and Min-Norm upon which final unified bias expression was developed [2]. 


\section{TABLE OF CONTENTS}

\section{PAGE}

ACKNOWLEDGEMENT. ..................... . . . . . . .

PREFACE........................ iv

LIST OF TABLES. ...................... . viii

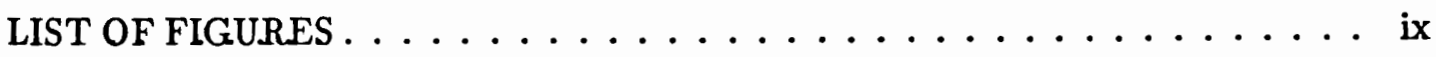

\section{CHAPTER}

I PRELIMINARIES .................... 1

I.1 Introduction $\ldots \ldots \ldots \ldots \ldots \ldots$

1.2 Thesis Outline .................... 2

I.3 Nomenclature ....................... 3

II DOA ESTIMATION ALGORITHMS REVIEW ........... 4

II.1 Problem Formulation of DOA Estimation . . . . . . . . . . 5

II.2 Subspace Decomposition ................. 6

II.3 Subspace Based DOA Estimation Algorithms . . . . . . . . 7

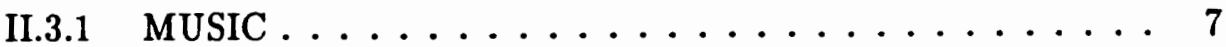

II.3.2 Min-Norm .................. 8

II.3.3 Root-MUSIC ................... 9

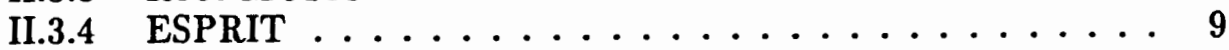

II.3.5 State-Space Realization ................ 10

II.3.6 Matrix-Pencil Method ................. 12

II.4 Classification of Subspace Based DOA Algorithms . . . . . . 13

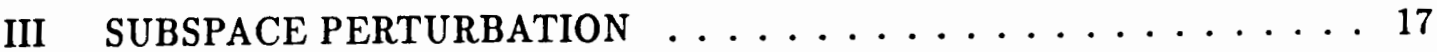

III.1 Introduction .................... 17 
III.2 Subspace Perturbation ................. 17

III.2.1 Review of the First-Order Subspace Perturbation . . . . . 20

III.2.2 Second-Order Subspace Perturbation . . . . . . . . 21

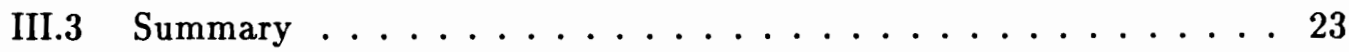

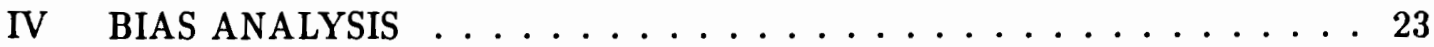

IV.1 Introduction $\ldots \ldots \ldots \ldots \ldots$

IV.2 Bias Analysis for Extrema-Search Algorithms . . . . . . . . . 24

IV.2.1 Bias for MUSIC Searching Algorithm .......... 26

IV.2.2 Bias for Min-Norm Searching Algorithm . . . . . . . . 27

IV.3 Bias Analysis of Polynomial Rooting Algorithms . . . . . . . . 28

IV.3.1 Bias for Root MUSIC ............... 30

IV.3.2 Bias for Root Min-Norm . . . . . . . . . . . . 31

IV.4 Equating of Extrema Searching Algorithms and Polynomial Rooting

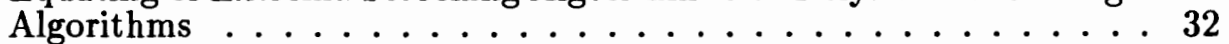

IV.5 Bias Analysis for Matrix-Shifting Algorithms .......... 33

IV.6 Numerical Simulations . . . . . . . . . . . . 35

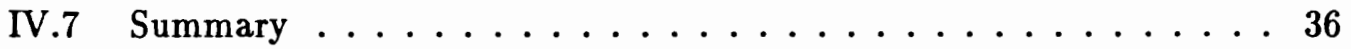

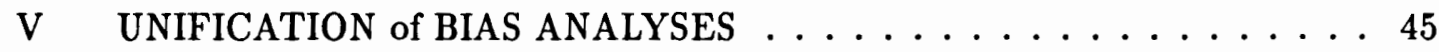

V.1 Introduction .................. 45

V.2 Unification of Bias Expressions .............. 45

V.3 Theoretical Study .................. 45

V.3.1 Bias, MSE and Variance vs. number of senors ...... 46

V.3.2 Bias, MSE and VAR vs. SNR ............. 47

V.3.3 Bias, VAR and MSE vs. snapshots ........... 48

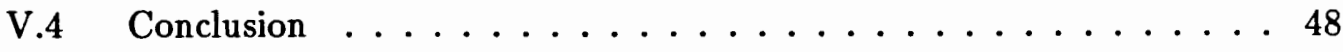

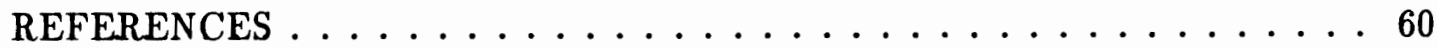




\section{APPENDICES}

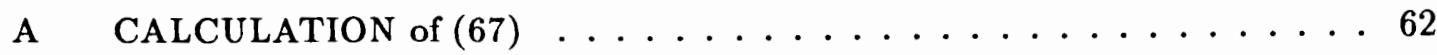

B BIAS DERIVATION FOR EXTREMA SEARCHING ALGORITHMS . . . 65

C BIAS DERIVATION FOR POLYNOMIAL ROOTING ALGORITHMS . . 75

D BIAS DERIVATION FOR MATRIX-SHIFTING ALGORITHMS . . . . . 85 


\section{LIST OF FIGURES}

FIGURE

PAGE

1 A Typical Array Scene .......................... 15

2 Subspace Decomposition: A Three-sehor Two-source Example [3] . . . . 16

3 Null-Spectrum Function of Extrema Search Approach . . . . . . . 38

4 Scattergram of Estimated Roots for Root-MUSIC . . . . . . . . . . 39

5 Scattergram of Estimated Roots for Min-Norm ... . . . . . . . . 40

6 Scattergram of Estimated Eigenvalues for ESPRIT ........... 41

7 Bias vs. SNR for MUSIC . . . . . . . . . . . . . . 42

8 Bias vs. SNR for Min-Norm .................. 43

9 Bias vs. SNR for ESPRIT ................... 44

10 Bias vs. Senors ....................... 51

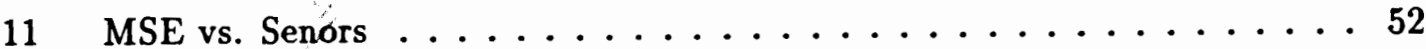

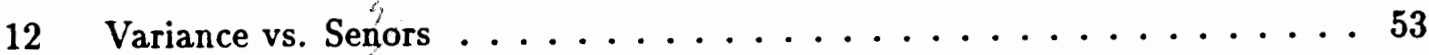

13 Bias vs. SNR .......................... 54

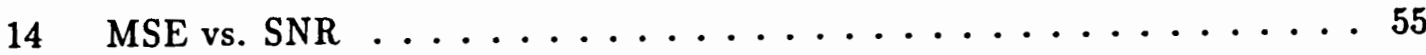

15 Variance vs. SNR ......................... 56

16 Bias vs. Snapshots ......................... 57

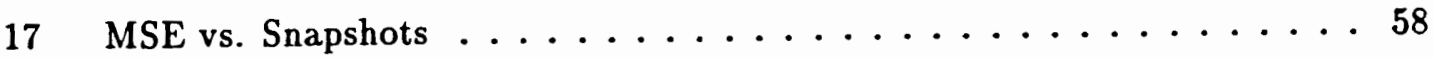

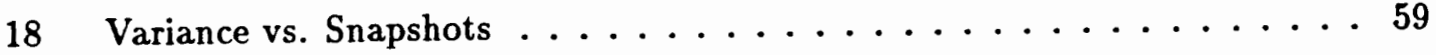




\section{LIST OF TABLES}

TABLE

PAGE

I MSE for MUSIC, Min-Norm and Root MUSIC, Root Min-Norm . . . . 33

II Weighting Matrix and Constant Specification for Bias Expression . . . . 50

III Weighting Matrix of MSE Expression . . . . . . . . . . . 50 


\section{CHAPTER I}

\section{PRELIMINARIES}

\section{I.1 INTRODUCTION}

Motivated by the increasing demands in applications such as radar detection, geophysical exploration, telecommunication and biomedical science, seíor array processing has been a very active research field for decades. One of the principle objective in senor array processing is to estimate direction of signals impinging simultaneously on an array of sey6rs. Many direction-of-arrival (DOA) estimation approaches have been proposed including pre-subspace approaches such as maximum entropy method and minimum variance method and subspace-based approaches. Among them, subspace-based DOA estimation methods are the currently most popular ones due to their relatively high resolution capability and low computational complexity. There exist different subspace algorithms which estimate DOAs by exploiting underlying signal models in different fashions. Therefore the performance analysis of these algorithms for the purpose of justification and comparison is important. Early studies on performance were based on simulations. Pioneered by Kaveh and Barabell [4], analytical evaluation of mean-squared DOA estimation error for subspace algorithms has attracted much excellent research (see [5] for a complete references). However, the bias behavior of subspace-based estimators remains largely unexplored, even though bias is of great importance in many applications such as predicting resolution threshold. This is because of theoretical difficulty and mathematical complexity involved in bias analysis. Kavelı and Barabell [4] addressed bias of estimated spectrum 
instead of estimated parameters. Wang and Wakefield [6] analyzed bias of estimated non-asymptotically, but their predicted bias does nọt match empirical results well even in the case where asymptotic measurements are used; $\mathrm{Xu}$ and Buckley [7] derived the bias and variance expressions for MUSIC and Min-Norm estimates using the asymptotic distribution of the estimated subspace vectors.

In this thesis, effort has been directed at developing a unified bias analysis of DOA estimation for subspace-based algorithms in terms of physical parameters as opposed to previous individual analysis expressed in parameters which depend on the specific data trials. Thus this analysis provides us insights into different algorithms to observe the direct relations between the performance of algorithms and signal measurement conditions.

\section{I.2 THESIS OUTLINE}

The rest of this thesis is organized as follows:

Chapter 2: formulating the DOA estimation problem and introduce the concept of subspace decomposition. Pertinent subspace-based DOA estimation approaches are summarized along with discussion on their disadvantages and advantages.

Chapter 3: Brief review of first-order subspace perturbation theory upon which second-order subspace perturbation is introduced and expanded. The subspace perturbation analysis established in this chapter provides common basis for further unified bias analysis in the following chapters.

Chapter 4: Bias performance analysis for subspace-based DOA estimation algorithms including extrema-searching algorithms, polynomial-rooting algorithms and matrix-shifting algorithms is developed based on finite data measurements. Accurate and concise bias expressions are presented in terms of physical parameters. Furthermore, the theoretical bias analysis is confirmed by simulations over a wide range of signal-to 
-noise ratios.

Chapter 5: A unified expression of DOA estimation bias with parameters specialized for different algorithms is presented. Based on that expression, we theoretically study and compare different subspace-based DOA estimation algorithms, from which some new insights into the performance of algorithms are drawn.

\section{I.3 NOMENCLATURE}

1. Matrix Operation:

$[\cdot]^{T}-$ transpose of matrix $[\cdot]$

$[\cdot]^{H}$ - complex-conjugate transpose of matrix $[\cdot]$

$[\cdot]^{\dagger}$ - left pseudo-inverse of matrix $[\cdot]$

$[\cdot]^{\uparrow}$ - matrix $[\cdot]$ with the first row deleted

$[\cdot]^{\downarrow}$ - matrix $[\cdot]$ with the last row deleted

$\Im[\cdot]$ - imaginary part of matrix $[\cdot]$

$\Re[\cdot]$ - real part of matrix $[\cdot]$

$\operatorname{Tr}[\cdot]$ - trace of matrix $[\cdot]$

2. Principle Symbols:

$K$ - number of sources

$L$ - number of sensors

$M$ - number of snapshots

$\theta_{k}-k$-th direction of arrival

$\sigma_{n}^{2}-$ noise power 
$\mathbf{a}\left(\theta_{k}\right)$ - array response vector

$\mathbf{e}_{k}^{H}-[0 \cdots 1 \cdots 0], 1$ in the $k$-th position

$\mathbf{e}_{1}^{H}-[1,0 \cdots 0], 1$ in the first position.

I - identity matrix

S - signal matrix

$\mathbf{N}$ - noise matrix

$\mathbf{A}(\theta)$ - array response matrix

$\boldsymbol{\Omega}_{o}$ - orthogonal-subspace projection matrix. $\boldsymbol{\Omega}_{o}=\mathbf{I}-\mathbf{A}\left(\mathbf{A}^{H} \mathbf{A}\right)^{-1} \mathbf{A}^{H}$

$\boldsymbol{\Omega}_{\boldsymbol{s}}$ - signal-subspace projection matrix. $\boldsymbol{\Omega}_{s}=\mathbf{A}\left(\mathbf{A}^{H} \mathbf{A}\right)^{-1} \mathbf{A}^{H}$

$\mathbf{R}_{\text {s }}$ - Source covariance matrix $\mathbf{W}$ - weighting matrix

$\Theta$ - location region of interest

3. Statistics Symbols:

$E\{\cdot\}$ - mathematical expectation of $\{\cdot\}$

$\stackrel{i}{=}-$ equal up to the order of $\|\mathbf{N}\|^{i}$

$[\cdot]_{1}-1$-th order approximation of noise-corrupted matrix $[\cdot]$

$[\cdot]_{2}-2$-th order approximation of noise-corrupted matrix $[\cdot]$ 


\section{CHAPTER II}

\section{DOA ESTIMATION ALGORITIIMS REVIEW}

\section{II.1 PROBLEM FORMULATION OF DOA ESTIMATION}

Consider $K$ signals (usually random processes) simultaneously incident on an array of $L$ sensors (Figure 1 is a typical array scene). The objective is to estimate direction of the arrival signals from the measurements taken simultaneously from all the seyors at $M$ different time instants.

The following assumptions are made about signal, noise and array structure.

- The $K$ signal wavefronts are narrow-band planar wavefronts with the same center wavelength $\lambda_{c}$ and are uncorrelated to each other.

- The number of signals is predetermined which satisfies $K<L$.

- The observation noise $n_{i}$ at each seyor is circular, stationary, additive complex white Gaussian process with zero mean and variance $\sigma_{n}^{2}$, uncorrelated to the signals.

- The array configuration is known and the response has been calibrated.

For the purpose of simplicity, we only consider uniform line array. Under the above assumptions, the signals arriving at the $i$-th selyor at time $t$ is

$$
\begin{aligned}
y_{i}(t) & =\sum_{k=1}^{K} e^{j \frac{2 \pi d}{\lambda_{c}}(i-1) \sin \theta_{k}} s_{k}(t) \\
& =\left(c^{j \frac{2 \pi d}{\lambda_{c}}(i-1) \sin \theta_{1}} \cdots c^{j \frac{2 \pi d}{\lambda_{c}}(i-1) \sin \theta_{K^{-}}}\right)\left(\begin{array}{c}
s_{1}(t) \\
\vdots \\
s_{K}(t)
\end{array}\right),
\end{aligned}
$$


where $s_{k}(t)$ is the $p$-th signal arrived at the angle $\theta_{k}$. The signals arrived at all sensors at time $t$ are

$$
\mathbf{y}(t)=\left(\begin{array}{ccc}
1 & \cdots & 1 \\
e^{j \frac{2 \pi d}{\lambda_{c}} \sin \theta_{1}} & \cdots & e^{j \frac{2 \pi d}{\lambda_{c}} \sin \theta_{K}} \\
\vdots & \ddots & \vdots \\
e^{j \frac{2 \pi d}{\lambda_{c}}(L-1) \sin \theta_{1}} & \cdots & e^{j \frac{2 \pi d}{\lambda_{c}}(L-1) \sin \theta_{K}}
\end{array}\right)\left(\begin{array}{c}
s_{1}(t) \\
\vdots \\
s_{K}(t)
\end{array}\right)
$$

or in vector form,

$$
\mathbf{y}(t) \stackrel{\text { def }}{=}\left[\mathbf{a}\left(\theta_{1}\right) \cdots \mathbf{a}\left(\theta_{K}\right)\right] \mathbf{s}(t) \stackrel{\text { def }}{=} \mathbf{A}(\theta) \mathbf{s}(t)
$$

where $\mathbf{A}(\theta)$ (and $\mathbf{a}(\theta)$ ) represents the array characteristics. Take $M$ snapshots of signals at each seyor to form the data matrix

$$
\mathbf{Y}=\left(\begin{array}{ccc}
y_{1}(1) & \cdots & y_{1}(M) \\
\vdots & \ddots & \vdots \\
y_{L}(M) & \cdots & y_{L}(M)
\end{array}\right)=\mathbf{A}(\theta) \mathbf{S}
$$

where

$$
\mathbf{S} \stackrel{\text { def }}{=}[\mathbf{s}(1) \cdots \mathbf{s}(M))]=\left(\begin{array}{ccc}
s_{1}(1) & \cdots & \left.s_{1}(M)\right) \\
\vdots & \ddots & \vdots \\
s_{K}(1) & \cdots & s_{K}(M)
\end{array}\right)
$$

The direction-finding problem can be accomplished by estimating $\left(\theta_{1} \cdots \theta_{K}\right)$ from array output $Y$.

\section{II.2 SUBSPACE DECOMPOSITION}

It is noticed from (4) that the matrix $\mathrm{A}(\theta)(L \times K)$ has rank $K$ and $\mathrm{S}(K \times M)$ has rank $K$, data matrix $\mathrm{Y}(L \times M)$ is a rank-deficient matrix, thus the subspace decomposition can be performed either directly on $Y$ by singular value decomposition (SVD) or on the sample covariance matrix $\tilde{\mathbf{R}}=\frac{1}{M} \tilde{\mathbf{Y}} \tilde{Y}^{H}$ by an eigenvalue decomposition. As shown in [8], these two approaches are essentially equivalent. For simplicity, we will employ SVD in our analysis. The subspace decomposition using SVD on the direct data matrix $Y$ is as follows:

$$
\mathbf{Y}=\mathbf{U} \boldsymbol{\Sigma} \mathbf{V}^{H}=\left(\begin{array}{ll}
\mathbf{U}_{s} & \mathbf{U}_{o}
\end{array}\right)\left(\begin{array}{cc}
\boldsymbol{\Sigma}_{s} & 0 \\
0 & 0
\end{array}\right)\left(\begin{array}{c}
\mathbf{V}_{s}^{H} \\
\mathbf{V}_{o}^{H}
\end{array}\right)
$$


where $\boldsymbol{\Sigma}$ are singular value matrix with decreasing singular values on the diagonal as

$$
\sigma_{1} \geq \cdots \geq \sigma_{K} \geq 0
$$

and $\mathrm{U}_{s}(L \times K)$ are singular vectors associated with the $K$ non-zero singular values while $\mathrm{U}_{o}(L \times(L-K))$ are singular vectors associated with $L-K$ zero singular values. There are two fundamental properties about the subspace upon which subspace-based DOA algorithms are established (see Figure 2).

- The vectors in $U_{s}$ span the signal subspace which is defined as the span of array manifold $\mathbf{A}(\theta)$ (the column space of $\mathbf{A}(\theta)$ ).

- The vectors in $U_{0}$ span the orthogonal subspace which is the orthogonal complement of signal subspace, i.e. the spanned array manifold $\mathbf{A}(\theta)$. The orthogonality between the orthogonal subspace and the array manifold can be expressed as

$$
\mathbf{a}\left(\theta_{k}\right)^{H} \mathrm{U}_{o}=0, \quad k=1, \cdots, K
$$

In a noisy environment, data matrix is perturbed resulting in the perturbation of the subspace as

$$
\tilde{\mathbf{Y}}=\mathbf{Y}+\mathbf{N}=\tilde{\mathbf{U}} \tilde{\mathbf{\Sigma}} \tilde{\mathbf{V}}^{H}=\left(\begin{array}{cc}
\tilde{\mathbf{U}}_{s} & \tilde{\mathbf{U}}_{o}
\end{array}\right)\left(\begin{array}{cc}
\tilde{\mathbf{\Sigma}}_{s} & 0 \\
0 & \tilde{\mathbf{\Sigma}}_{o}
\end{array}\right)\left(\begin{array}{c}
\tilde{\mathbf{V}}_{s}^{H} \\
\tilde{\mathbf{V}}_{o}^{H}
\end{array}\right)
$$

It was proved in [8] that orthogonal subspace is asymptotically orthogonal to the signal manifold.

\section{II.3 SUBSPACE BASED DOA ESTIMATION ALGORITHMS}

\section{II.3.1 MUSIC}

MUSIC (MUitiple SIgnal Classification algorithm) was proposed by Schmidt [9]. It utilizes the orthogonality between signal subspace and orthogonal subspace to perform 
an one-dimensional search for $K$ zeros over the null-spectrum

$$
P_{M U}(\theta)=\mathbf{a}(\theta)^{H} \mathbf{U}_{o} \mathbf{U}_{o}^{H} \mathbf{a}(\theta)
$$

when noise perturbs, null-spectrum $P_{M U}(\theta)$ reaches $K$ minima around the true DOAs.

In this and future equations, the symbol $\theta$ without a subscript is a scalar variable which represents a possible direction of arrival, while the subscripted symbol $\theta_{k}, \quad k=$ $1, \cdots, K$ is referred to the actual directions of arrival in the noise-free data. The advantage of MUSIC is that there is no constraint on the geometry of the senor array as long as it is known or calibrated. Yet the algorithms is achieved at the expense of considerable computation and storage through the search over directions of interesting for DOAs. Up to date, MUSIC is the most popular and most cited work. It has been widely studied and many modified versions have been proposed such as weighted MUSIC and Root-MUSIC.

\section{I1.3.2 Min-Norm}

Min-Norm (Minimum-Norm algorithm) was introduced by Kumaresan and Tufts [10]. The motivation of Min-Norm was to identify a single vector $\mathbf{d}$ in the span of orthogonal subspace with unity first element and minimum Euclidian norm. This vector is constructed by linear combination of all $L-K$ vectors in $\mathrm{U}_{o}$ as

$$
\mathbf{d}=\mathbf{U}_{o} \frac{\mathbf{c}}{\|\mathbf{c}\|^{2}}
$$

where $c^{H}$ is the first row of $U_{o}$. For uniform line array, DOA estimation is performed through polynomial rooting

$$
D(z)=\mathbf{a}(z) \mathbf{d}=\prod_{i=1}^{L-1}\left(1-r_{i}^{*} z\right) .
$$

The $K$ roots on the unit circle with $r_{i}=e^{j \frac{2 \pi d}{\lambda_{c}} \sin \theta_{i}} \quad i=1, \cdots, K$ contain the DOA information while the rest $L-K-1$ are regarded as extraneous roots. In the noisy case, 
$K$ roots with the largest amplitudes are chosen as the signal-roots and the rest are referred as noise roots.

Min-Norm is also applicable to arbitrary array geometry as proved by $\mathrm{Li}$ [11] by searching for the $K$ zeros of the null-spectrum over $\theta$

$$
P_{M N}(\theta)=\left|\mathbf{a}^{H}(\theta) \mathbf{d}\right|^{2}
$$

Compared to MUSIC, Min-Norm requires less computation and storage. But the algorithm provides DOA estimates with variances comparatively larger than those of MUSIC.

\section{II.3.3 Root-MUSIC}

Root-MUSIC [12] only applies to uniform line array. It forms and roots the nullspectrum polynomial

$$
P_{R M}(z)=\mathrm{a}\left(z^{-1}\right)^{T} \mathbf{U}_{o} \mathbf{U}_{o}^{H} \mathbf{a}(z)=A \prod_{i=1}^{L-1}\left(1-r_{i} z^{-1}\right)\left(1-r_{i}^{*} z\right)
$$

Root-MUSIC, unlike Min-Norm, always chooses the $P$ roots with largest amplitudes insides the unit-circle. This choice results in a bias in the radial direction of the estimated roots since when noise presents the signal-root will be perturbed inside and outside the unit-circle. However, DOA estimates are only functions of the angles of the roots, not the radii. Thus the radius error does not affect the DOA estimates obtained by Root-MUSIC.

\section{II.3.4 ESPRIT}

ESPRIT (Estimation of Signal Parameters via Rotational Invariant Techniques) [13] utilizes two identical sub arrays which are physically displaced from each other by a known displacement $\Delta$ to obtain a shift-invariant structure. When a uniform line array is used, we can choose the first $L-1$ sensors as first sub array and last $L-1$ sensors as 
second sub array for the maximum sub array apertures (then $\Delta=d$ ). The shift-invariance can be expressed using array manifold

$$
\mathbf{A}^{\downarrow} \mathbf{D}=\mathbf{A}^{\uparrow}
$$

where $\mathbf{A}^{\downarrow}$ and $\mathbf{A}^{\dagger}$ are the first $L-1$ rows and last $L-1$ rows of $\mathbf{A}$. The diagonal matrix $\mathrm{D}$ has the diagonal element $\lambda_{k}=e^{j \frac{2 \pi d}{\lambda_{c}} \sin \theta_{k}}$. In practice, we obtain $\mathbf{D}$ as the eigenvalue matrix of $\mathbf{F}_{e s}$

$$
\mathbf{U}_{s}^{\dagger} \mathbf{F}_{e s}=\mathbf{U}_{s}^{\uparrow}
$$

where $U_{s}^{\dagger}$ and $U_{s}^{!}$are the first $L-1$ rows and last $L-1$ rows of $U_{s}$, respectively. $F_{e s}$ can be obtained as

$$
\mathbf{F}_{e s}=\mathbf{U}_{s}^{\dagger} \mathbf{U}_{s}^{\dagger}
$$

$\mathrm{D}$ is related to $\mathbf{F}_{\text {es }}$ through an eigenvalue decomposition.

$$
\mathbf{D}=\mathbf{L}^{-1} \mathbf{F}_{e s} \mathbf{L}
$$

ESPRIT has certain advantages over MUSIC:

- It does not require knowledge of the array geometry and element characteristics.

- It mitigates the computational and storage requirement of MUSIC.

However, there are still strong constraints of array geometry: the corresponding sensors in each subarray must have identical characteristics, and they must be equally displaced, in parallel and in the same plane.

\section{II.3.5 State-Space Realization}

The state space includes a covariance approach and a direct-data approach [14, $15,16,17]$ which are equivalent. 
The state space approach can be used with an ESPRIT-type array, but what follows is based on a uniform line array for the purpose of analysis.

Recall

$$
\begin{aligned}
y_{i}(t) & =\sum_{k=1}^{K} e^{j \frac{2 \pi d}{\lambda_{c}}(i-1) \sin \theta_{k}} s_{k}(t) \\
& =\left(e^{j \frac{2 \pi d}{\lambda_{c}}(i-1) \sin \theta_{1}} \cdots e^{j \frac{2 \pi d}{\lambda_{c}}(i-1) \sin \theta_{K}}\right) \mathbf{s}(t) .
\end{aligned}
$$

A state-space model for a plane-wave propagating in a sensor array can be derived as

$$
\begin{aligned}
\mathbf{x}_{i+1}(t) & =\mathbf{F} \mathbf{x}_{i}(t) \\
y_{i}(t) & =\mathbf{h} \mathbf{x}_{i}(t)
\end{aligned}
$$

or

$$
y_{i}(t)=\mathbf{h} \mathbf{F}^{i-1} \mathbf{x}_{1}(t)
$$

where the $\mathbf{x}$ is the state-vector with the initial value $\mathbf{x}_{1}(t)=\mathbf{s}(t)$ and $\mathbf{h}=[1, \cdots, 1]$ is row-vector of sensor gains, and

$$
\mathbf{F}=\left(\begin{array}{cccc}
e^{j \frac{2 \pi d}{\lambda_{c}} \sin \theta_{1}} & 0 & \cdots & 0 \\
0 & e^{j \frac{2 \pi d}{\lambda_{c}} \sin \theta_{2}} & \ddots & \vdots \\
\vdots & \ddots & \ddots & 0 \\
0 & \cdots & 0 & e^{j \frac{2 \pi d}{\lambda_{c}} \sin \theta_{K}}
\end{array}\right)
$$

The diagonal entries of $\mathbf{F}$ contain the information of the arrival direction. The data matrix is formed and factored as follows

$$
\begin{aligned}
\mathbf{Y} & =\left(\begin{array}{ccc}
y_{1}(1) & \cdots & y_{1}(M) \\
\vdots & \ddots & \vdots \\
y_{L}(M) & \cdots & y_{L}(M)
\end{array}\right) \\
& =\left(\begin{array}{c}
\mathbf{h} \\
\mathbf{h F} \\
\vdots \\
\mathbf{h F}
\end{array}\right)(\mathbf{s}(0) \cdots \mathbf{s}(M)) \stackrel{\text { def }}{=} \mathbf{O C} .
\end{aligned}
$$


$\mathbf{O}$ and $\mathbf{C}$ are respectively the observability matrix and controllability matrix. Let $\mathbf{O}^{\dagger}$ be the $O$ except the first row and $O^{\prime}$ be $O$ except the last row. From (23), it is clear that the following shift-invariance is true

$$
\mathbf{O}^{\downarrow} \mathbf{F}=\mathbf{O}^{\uparrow}
$$

Therefore a transition matrix $\mathbf{F}$ is obtained from (24)

$$
\mathbf{F}=\mathbf{O}^{\dagger} \mathbf{O}^{\dagger}
$$

In general, the factors of the data matrix are obtained from a singular value decomposition (6). By taking the principal components and using the concept of state-variable balancing

$$
\mathbf{O}=\mathrm{U}_{s} \Sigma_{s}^{\frac{1}{2}}
$$

and then $\mathbf{F}$ can be solved for as

$$
\mathbf{F}=\left(\mathbf{U}_{s}^{\dagger} \Sigma_{s}^{\frac{1}{2}}\right)^{\dagger} \mathbf{U}_{s}^{\dagger} \Sigma_{s}^{\frac{1}{2}}
$$

We can see that ESPRIT and SSR under uniform line array has the same DOA estimates.

\section{II.3.6 Matrix-Pencil Method}

The ideas that the Matrix-Pencil Method [18, 19, 20] and ESPRIT method exploit are identical despite the fact that each of them has many versions. ESPRIT was proposed from an array geometrical design point of view while the Matrix-Pencil method was developed in applying results of linear algebra to the problem of direction-of-arrival estimation. In this sense, we can say that the Matrix-Pencil Method is a generalized version of State-Space Realization and ESPRIT. The Matrix-Pencil Method forms two matrices with certain relationship in between, then solves the generalized eigenvalue problem of matrix-pencil by employing that relation. Three versions of the Matrix-Pencil Method summarized in [21] are as follows: 
1. A matrix pencil is

$$
\mathbf{X}-\lambda \mathbf{Z}
$$

where $\lambda$ can be solved from a generalized eigenvalue problem

$$
\left(\mathbf{Z}^{\dagger} \mathbf{X}-\lambda_{k}\right) \mathbf{q}_{k}=0
$$

2. Form a data matrix

$$
\mathbf{Y}=\left(\begin{array}{l}
\mathbf{X} \\
\mathbf{Z}
\end{array}\right)
$$

and take the principal components from the singular value decomposition to $Y$, we have

$$
\mathbf{Y}=\mathbf{U} \boldsymbol{\Sigma} \mathbf{V}^{\mathbf{H}}=\left(\begin{array}{c}
\mathbf{U}_{\boldsymbol{x}} \\
\mathbf{U}_{z}
\end{array}\right) \boldsymbol{\Sigma} \mathbf{V}^{\mathbf{H}} .
$$

A matrix pencil is then formed as

$$
\mathbf{U}_{s x}-\lambda \mathbf{U}_{s z}
$$

and $\lambda$ is solved as an eigenvalue of $F$ as

$$
\mathrm{U}_{s z} \mathbf{F}=\mathrm{U}_{s x}
$$

For this reason, we combine the analysis of the Matrix-Pencil method into the analysis of ESPRIT.

3. A total least-square approach [21] (which is also applicable to ESPRIT and State-Space Realization).

\section{II.4 CLASSIFICATION OF SUBSPACE BASED DOA ALGORITHMS}

The subspace-based DOA algorithms can be classified into three approaches according to numerical procedure which each algorithm follows.

- Extrema-Searching Approach: MUSIC and Min-Norm searching algorithms. 
- Polynomial-Rooting Approach: Min-Norm and Root-MUSIC.

- Matrix-Shifting Approach: ESPRIT, State-Space Realization and Matrix -Pencil method. 


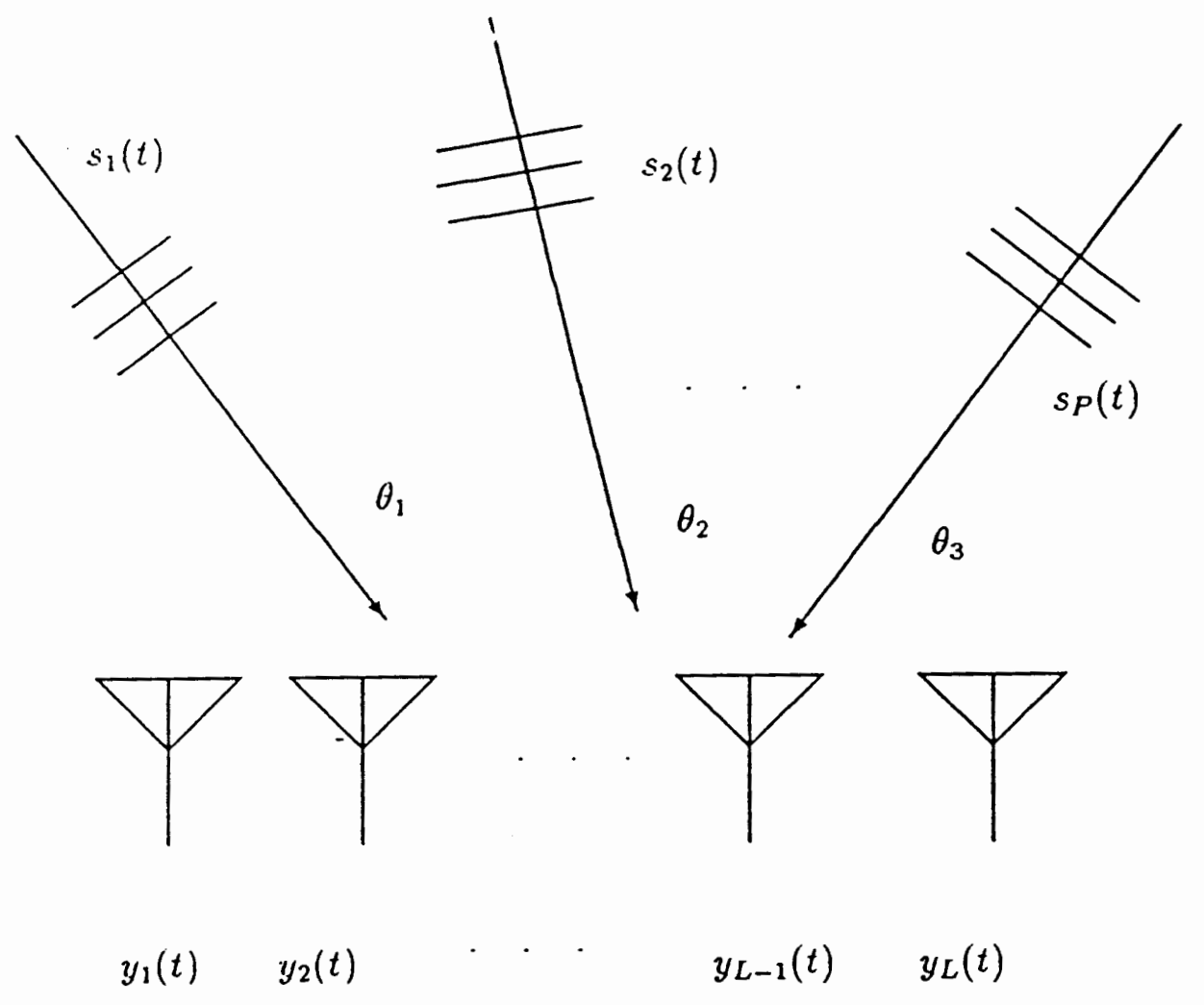

Figure 1. A Typical Array Scene. 


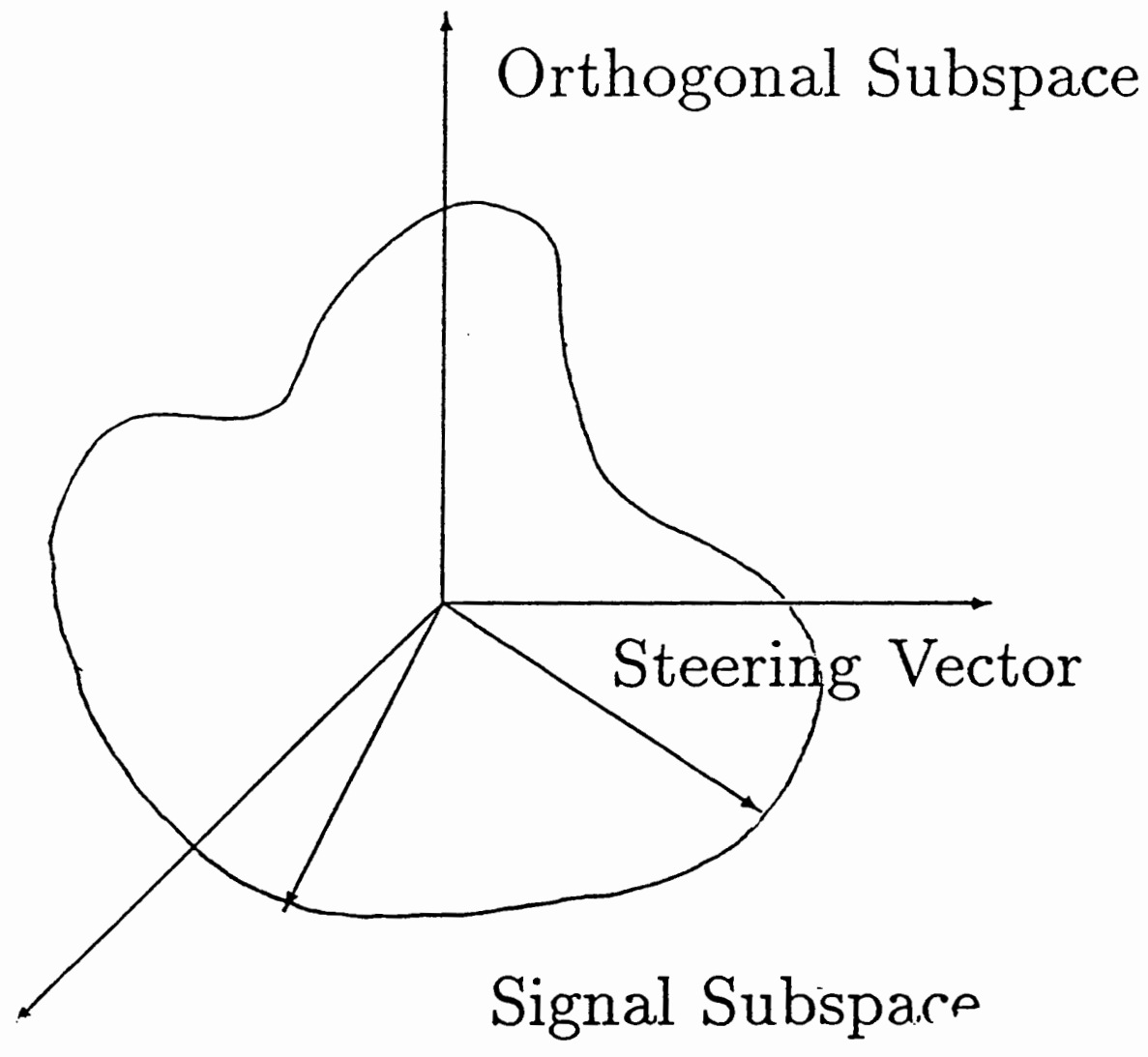

Figure 2. Subspace Decomposition: A Three-senor Two-source Example [3]. 


\section{CIIAPTER III}

\section{SUBSPACE PERTURBATION}

\section{III.I INTRODUCTION}

All the subspace processing algorithms are based on utilizing the properties of the subspaces obtained from subspace decomposition. When the data is corrupted by noise, the subspaces obtained from SVD on the data are perturbed. In [s], Li extensively studied the first-order subspace perturbations upon which a unified formula for mean-square error of subspace algorithms is derived successfully. Unfortunately, zero bias results from the first-order subspace perturbations analysis, which indicates higher order perturbations should be included for an accurate bias expression. In this chapter, the analysis for second-order subspace perturbations is developed, which provides common foundation for bias analysis for different algorithms.

\section{III.2 SUBSPACE PERTURBATION}

Various data perturbations are always presented in practice which result in the perturbation of estimated subspaces. The perturbed data matrix can be written as

$$
\dot{\mathbf{Y}}=\mathbf{Y}+\mathbf{N}
$$

where $\mathrm{N}$ is the observation noise which assumed to be circular Ciaussian with zero mean. The subspace decomposition of perturbed data by SVD is

$$
\tilde{\mathbf{Y}}=\tilde{\mathbf{U}} \tilde{\mathbf{\Sigma}} \dot{\mathbf{V}}^{H}=\left(\begin{array}{ll}
\tilde{U}_{s} & \tilde{\mathrm{U}}_{o}
\end{array}\right)\left(\begin{array}{cc}
\tilde{\mathbf{\Sigma}}_{s} & 0 \\
0 & \tilde{\mathbf{\Sigma}}_{o}
\end{array}\right)\left(\begin{array}{c}
\tilde{\mathbf{V}}_{s}^{H} \\
\tilde{\mathbf{V}}_{o}^{H}
\end{array}\right) .
$$


We can now write

$$
\tilde{\mathbf{U}}_{o}=\mathbf{U}_{o}+\Delta \mathbf{U}_{o} \text { and } \tilde{\mathbf{U}}_{s}=\mathbf{U}_{s}+\Delta \mathbf{U}_{s}
$$

where $\Delta U_{o}$ and $\Delta U_{s}$ are the perturbations in the estimated orthogonal- and signalsubspaces. The following lemma was proved in [5].

Lemma : The perturbed orthogonal subspace is spanned by $U_{o}+U_{s} Q$ and the perturbed signal-subspace is spanned by $\mathbf{U}_{s}+\mathbf{U}_{o} \mathbf{P}$, where $\mathbf{P}$ and $\mathbf{Q}$ are matrices whose norms are of the order of $\|\mathbf{N}\|$. The matrix norm can be any submultiplicative norm such as the Euclidean 2-norm or the Frobenius norm.

Noticing that

$$
\begin{aligned}
& \tilde{\mathbf{U}}_{o}^{H} \tilde{\mathbf{U}}_{o}=\left(\mathbf{U}_{o}+\mathbf{U}_{s} \mathbf{Q}\right)^{H}\left(\mathbf{U}_{o}+\mathbf{U}_{s} \mathbf{Q}\right)=\mathbf{I}+\mathbf{Q}^{H} \mathbf{Q} \\
& \tilde{\mathbf{U}}_{s}^{H} \tilde{\mathbf{U}}_{s}==\left(\mathbf{U}_{s}+\mathbf{U}_{o} \mathbf{P}\right)^{H}\left(\mathbf{U}_{s}+\mathbf{U}_{o} \mathbf{P}\right)=\mathbf{I}+\mathbf{P}^{H} \mathbf{P},
\end{aligned}
$$

which imply that $U_{o}+U_{s} \mathbf{Q}$ and $U_{s}+U_{o} P$ are not orthonormal. The orthonormal basis of perturbed subspaces are thus given as

$$
\begin{aligned}
& \tilde{\mathbf{U}}_{o}=\left(\mathbf{U}_{o}+\mathbf{U}_{s} \mathbf{Q}\right)\left(\mathbf{I}+\mathbf{Q}^{H} \mathbf{Q}\right)^{-\frac{1}{2}} \\
& \tilde{\mathbf{U}}_{s}=\left(\mathbf{U}_{s}+\mathbf{U}_{o} \mathbf{P}\right)\left(\mathbf{I}+\mathbf{P}^{H} \mathbf{P}\right)^{-\frac{1}{2}} .
\end{aligned}
$$

The relationship between $\mathbf{P}$ and $\mathbf{Q}$ can be derived using the orthogonality between the perturbed orthogonal- and signal- subspaces

$$
\begin{aligned}
\left.\left(\mathbf{I}+\mathbf{Q}^{H} \mathbf{Q}\right)^{-\frac{1}{2}}\left(\mathbf{U}_{o}+\mathbf{U}_{s} \mathbf{Q}\right)^{H}\left(\mathbf{U}_{s}+\mathbf{U}_{o} \mathbf{P}\right)\right)\left(\mathbf{I}+\mathbf{P}^{H} \mathbf{P}\right)^{-\frac{1}{2}} & =0 \\
\left(\mathbf{U}_{o}+\mathbf{U}_{s} \mathbf{Q}\right)^{H}\left(\mathbf{U}_{s}+\mathbf{U}_{o} \mathbf{P}\right) & =0 \\
\mathbf{P}+\mathbf{Q}^{H} & =0 .
\end{aligned}
$$

Similarly, it can be proved that

$$
\tilde{\mathbf{V}}_{o}=\left(\mathbf{V}_{o}+\mathbf{V}_{\boldsymbol{s}} \overline{\mathbf{Q}}\right)\left(\mathbf{I}+\overline{\mathbf{Q}}^{H} \overline{\mathbf{Q}}\right)^{-\frac{1}{2}}
$$




$$
\tilde{\mathbf{V}}_{s}=\left(\mathbf{V} s+\mathbf{V}_{o} \overline{\mathbf{P}}\right)\left(\mathbf{I}+\overline{\mathbf{P}}^{H} \overline{\mathbf{P}}\right)^{-\frac{1}{2}}
$$

where $\overline{\mathbf{Q}}$ and $\overline{\mathbf{P}}$ are matrices whose norms are also in the order of $\|\mathbf{N}\|$.

In the bias analysis, we are interested in the first-order and the second order approximations of subspace perturbations. Define []$_{1}$ and $[\cdot]_{2}$ as the first- and secondorder approximations of $[\cdot]$ in term of data perturbation $\mathbf{N}$, respectively, and use the notation $\stackrel{\dot{i}}{=}$ to mean "equal up to the order of $\|\mathbf{N}\|^{i n}$. The first-order approximation of orthogonal perturbed subspace is obtained by first-order expansion of (37) which is illustrated as follows:

Considering

$$
\left(\mathbf{I}+\mathbf{Q}^{H} \mathbf{Q}\right)^{-\frac{1}{2}}=\mathbf{I}-\frac{1}{2} \mathbf{Q}^{H} \mathbf{Q}+\cdots
$$

Keep (42) up to zeroth order and substitute it into (37) so as to expand the perturbation expansion to first-order,

$$
\tilde{\mathbf{U}}_{o 1}=\mathbf{U}_{o}+\mathbf{U}_{s} \mathbf{Q}_{1}
$$

Compare (43) with (35) to obtain first-order orthogonal subspace perturbation as

$$
\Delta \mathbf{U}_{o 1}=\mathbf{U}_{\mathbf{s}} \mathbf{Q}_{1}
$$

Similarly, the first-order signal subspace perturbation is expressed as

$$
\Delta \mathbf{U}_{s 1}=\mathbf{U}_{o} \mathbf{P}_{1}
$$

The second-order approximation of perturbed orthogonal subspace is obtained by keeping (42) up to second-order as

$$
\left(\mathbf{I}+\mathbf{Q}^{H} \mathbf{Q}\right)^{-\frac{1}{2}} \stackrel{2}{=} \mathbf{I}-\frac{1}{2} \mathbf{Q}_{1}^{H} \mathbf{Q}_{\mathbf{1}}
$$

Substitute (46) into (37) then keep the equation up to second-order

$$
\tilde{\mathbf{U}}_{o 2} \stackrel{2}{=}\left(\mathbf{U}_{o}+\mathbf{U}_{s} \mathbf{Q}_{2}\right)\left(\mathbf{I}-\frac{1}{2} \mathbf{Q}_{1}^{H} \mathbf{Q}_{1}\right)
$$


Delete the fourth-order term in (47) which yields

$$
\tilde{\mathbf{U}}_{o 2}=\mathbf{U}_{o}+\mathbf{U}_{s} \mathbf{Q}_{2}-\frac{1}{2} \mathbf{U}_{o} \mathbf{Q}_{1}^{H} \mathbf{Q}_{1}
$$

From (35) and (48), the second-order orthogonal subspace perturbation is given as

$$
\Delta \mathbf{U}_{o 2}=\mathbf{U}_{s} \mathbf{Q}_{2}-\frac{1}{2} \mathbf{U}_{o} \mathbf{Q}_{1}^{H} \mathbf{Q}_{1}
$$

Through the same step, the second-order signal subspace perturbation is given as

$$
\Delta \mathbf{U}_{s 2}=\mathbf{U}_{o} \mathbf{P}_{2}-\frac{1}{2} \mathbf{U}_{s} \mathbf{P}_{1}^{H} \mathbf{P}_{1}
$$

\section{III.2.1 Review of the First-Order Subspace Perturbation}

In this section, we will review the previous work on the first-order subspace perturbations developed by $\mathrm{Li}$ and Vaccaro for future reference in bias analysis of DOA estimation algorithms.

Pre-multiply (34) with $\tilde{\mathrm{U}}_{0}^{H}$

$$
\tilde{\mathbf{U}}_{o}^{H} \tilde{\mathbf{Y}}=\tilde{\mathbf{\Sigma}}_{o} \tilde{\mathbf{V}}_{o}^{H}
$$

Using (35) and (44) and the fact that $\tilde{\boldsymbol{\Sigma}}_{o}=\Delta \boldsymbol{\Sigma}_{o}$ (since the noise-free value of $\boldsymbol{\Sigma}_{o}$ is $\left.\Sigma_{o}=0\right),(51)$ can be written as

$$
\left(\mathbf{U}_{o}+\mathbf{U}_{\mathbf{s}} \mathbf{Q}_{1}\right)^{H}(\mathbf{Y}+\mathbf{N})=\Delta \mathbf{\Sigma}_{o}\left(\mathbf{V}_{o}+\mathbf{V}_{\mathbf{s}} \overline{\mathbf{Q}}_{\mathbf{1}}\right)^{H}
$$

Using the fact that $\mathrm{U}_{o}^{H} \mathbf{Y}=0$ to obtain

$$
\mathbf{U}_{o}^{H} \mathbf{N}+\mathbf{Q}_{1}^{H} \mathbf{U}_{s}^{H} \mathbf{Y}+\mathbf{Q}_{1}^{H} \mathbf{U}_{s}^{H} \mathbf{N}=\Delta \mathbf{\Sigma}_{o} \mathbf{V}_{o}^{H}+\Delta \boldsymbol{\Sigma}_{o} \overline{\mathbf{Q}}_{1}^{H} \mathbf{V}_{s}^{H}
$$

Next, post-multiply (53) with $\mathrm{V}_{s}$, we have

$$
\mathbf{U}_{o}^{H} \mathbf{N} \mathbf{V}_{s}+\mathbf{Q}_{1}^{H} \mathbf{\Sigma}_{s}+\mathbf{Q}_{1}^{H} \mathbf{U}_{s}^{H} \mathbf{N} \mathbf{V}_{s}=\Delta \mathbf{\Sigma}_{o} \overline{\mathbf{Q}}_{1}^{H}
$$


The first-order approximation of $\mathbf{Q}$ is then obtained from the first-order expansion of (54) as

$$
\mathbf{U}_{o}^{H} \mathbf{N V}_{s}+\mathbf{Q}_{1}^{H} \boldsymbol{\Sigma}_{s} \stackrel{1}{=} 0
$$

which gives

$$
\mathbf{Q}_{1}=-\boldsymbol{\Sigma}_{s}^{-1} \mathbf{V}_{s}^{H} \mathbf{N}^{H} \mathbf{U}_{o}
$$

Using (39), $\mathbf{P}_{1}$ can be obtained as

$$
\mathbf{P}_{1}=\mathbf{U}_{o}^{H} \mathrm{NV}_{s} \boldsymbol{\Sigma}_{s}^{-1}
$$

So far, the first-order subspace perturbations are obtained as

$$
\begin{gathered}
\Delta \mathbf{U}_{o 1}=\mathbf{U}_{s} \mathbf{Q}_{1}=-\mathbf{U}_{s} \boldsymbol{\Sigma}_{s}^{-1} \mathbf{V}_{s}^{H} \mathbf{N}^{H} \mathbf{U}_{o} \\
\Delta \mathbf{U}_{s 1}=\mathbf{U}_{0} \mathbf{P}_{1}=\mathbf{U}_{o} \mathbf{U}_{o}^{H} \mathbf{N} \mathbf{V}_{s} \boldsymbol{\Sigma}_{s}^{-1} .
\end{gathered}
$$

\section{III.2.2 Second-Order Subspace Perturbation}

In this section, we will derive explicit expressions for subspace perturbations which are valid up to the second-order with respect to data perturbation $\mathbf{N}$. The derivation here is also applicable to obtaining higher order subspace perturbations.

We begin from (51). Using (37), (40) and the fact that $\tilde{\boldsymbol{\Sigma}}_{o}=\Delta \boldsymbol{\Sigma}_{o}$ (since the noise-free value of $\boldsymbol{\Sigma}_{o}$ is $\left.\boldsymbol{\Sigma}_{o}=0\right),(51)$ can be written as

$$
\left(\mathbf{I}+\mathbf{Q}^{H} \mathbf{Q}\right)^{-\frac{1}{2}}\left(\mathbf{U}_{o}+\mathbf{U}_{s} \mathbf{Q}\right)^{H}(\mathbf{Y}+\mathbf{N})=\Delta \boldsymbol{\Sigma}_{o}\left(\mathbf{I}+\overline{\mathbf{Q}}^{H} \overline{\mathbf{Q}}\right)^{-\frac{1}{2}}\left(\mathbf{V}_{o}+\mathbf{V}_{s} \overline{\mathbf{Q}}\right)^{H}
$$

Using the fact that $\mathrm{U}_{o}^{H} \mathrm{Y}=0$, we get

$$
\left(\mathbf{I}+\mathbf{Q}^{H} \mathbf{Q}\right)^{-\frac{1}{2}}\left(\mathbf{U}_{o}^{H} \mathbf{N}+\mathbf{Q}^{H} \mathbf{U}_{s}^{H} \mathbf{Y}+\mathbf{Q}^{H} \mathbf{U}_{s}^{H} \mathbf{N}\right)=\Delta \boldsymbol{\Sigma}_{o}\left(\mathbf{I}+\overline{\mathbf{Q}}^{H} \overline{\mathbf{Q}}\right)^{-\frac{1}{2}}\left(\mathbf{V}_{o}+\mathbf{V}_{s} \overline{\mathbf{Q}}\right)^{H}
$$

$Q_{2}$ can be obtained by expanding (61) into second-order equation

$$
\mathbf{U}_{o}^{H} \mathbf{N}+\mathbf{Q}_{2}^{H} \mathbf{U}_{s}^{H} \mathbf{Y}+\mathbf{Q}_{1}^{H} \mathbf{U}_{s}^{H} \mathbf{N} \stackrel{2}{=} \Delta \boldsymbol{\Sigma}_{o 2} \mathbf{V}_{o}^{H}+\Delta \boldsymbol{\Sigma}_{o 1} \overline{\mathbf{Q}}_{1}^{H} \mathbf{V}_{s}^{H}
$$


Again, post-multiply (62) with $\mathrm{V}_{s}$,

$$
\mathbf{U}_{o}^{H} \mathbf{N} \mathbf{V}_{s}+\mathbf{Q}_{2}^{H} \boldsymbol{\Sigma}_{s}+\mathbf{Q}_{1}^{H} \mathbf{U}_{s}^{H} \mathbf{N V}_{s} \stackrel{2}{=} \Delta \boldsymbol{\Sigma}_{o 1} \overline{\mathbf{Q}}_{1}^{H}
$$

where $\Delta \boldsymbol{\Sigma}_{o 1}$ can be obtained by post-multiplying $V_{o}$ to both sides of (62) and expanding it into first-order equation as

$$
\Delta \mathbf{\Sigma}_{o 1}=\mathbf{U}_{o}^{H} \mathbf{N V}_{o}
$$

and $\overline{\mathbf{Q}}_{1}$ can be obtained by pre-multiplying $\mathbf{U}_{s}^{H}$ and post-multiplying $\tilde{\mathbf{V}}_{o}$ to $\tilde{\mathbf{Y}}$, then going through similar derivation. We have

$$
\overline{\mathbf{Q}}_{1}=-\boldsymbol{\Sigma}_{s}^{-1} \mathbf{U}_{s}^{H} \mathbf{N} \mathbf{V}_{o}
$$

Substitute (56), (64), and (65) into (63), we obtain

$$
\begin{aligned}
\mathbf{Q}_{2}= & -\boldsymbol{\Sigma}_{s}^{-1} \mathbf{V}_{s}^{H} \mathbf{N}^{H} \mathbf{U}_{o}+\boldsymbol{\Sigma}_{s}^{-1} \mathbf{V}_{s}^{H} \mathbf{N}^{H} \mathbf{U}_{s} \boldsymbol{\Sigma}_{s}^{-1} \mathbf{V}_{s}^{H} \mathbf{N}^{H} \mathbf{U}_{o} \\
& -\boldsymbol{\Sigma}_{s}^{-2} \mathbf{U}_{s}^{H} \mathbf{N} \mathbf{V}_{o} \mathbf{V}_{o}^{H} \mathbf{N}^{H} \mathbf{U}_{o} .
\end{aligned}
$$

Again $\mathbf{P}_{2}$ can be obtained from (39). An important statistical quantity is $E\left(\mathbf{Q}_{2}\right)$. We notice that if the observation noise is circular Gaussian with zero-mean, then the expectation of first term in (66) is equal to zero because of zero-mean; The expectation of second term is equal to zero because of circularity [22]. The expectation of the third term is also zero because

$$
E\left(\boldsymbol{\Sigma}_{s}^{-2} \mathbf{U}_{s}^{H} \mathbf{N V} \mathbf{V}_{o}^{H} \mathbf{N}^{H} \mathbf{U}_{o}\right)=\operatorname{Tr}\left(\mathbf{V}_{o} \mathbf{V}_{o}^{H}\right) \boldsymbol{\Sigma}_{s}^{-2} \mathbf{U}_{s}^{H} \mathbf{U}_{o} \sigma_{n}^{2}
$$

where $\mathbf{U}_{s}^{H} \mathbf{U}_{o}=0, T r$ stands for matrix trace. The detailed derivation is in Appendix A. Therefore we conclude

$$
E\left(\mathbf{Q}_{2}\right)=E\left(-\mathbf{P}_{2}^{H}\right)=0
$$


Now we can study the statistics properties of subspace perturbations by plugging (68), (56), (57) and (136) in the Appendix A into (49) and (50), respectively,

$$
\begin{gathered}
E\left(\Delta \mathrm{U}_{o 2}\right)=-\frac{1}{2} \mathrm{U}_{o} \mathrm{U}_{o}^{H} E\left(\mathrm{NV}_{s} \Sigma_{s}^{-2} \mathrm{~V}_{s}^{H} \mathrm{~N}^{H}\right) \mathrm{U}_{o}=-\frac{1}{2} \mathrm{U}_{o} \operatorname{Tr}\left(\mathrm{V}_{s} \Sigma_{s}^{-2} \mathrm{~V}_{s}^{H}\right) \sigma_{n}^{2} \\
E\left(\Delta \mathrm{U}_{s 2}\right)=-\frac{1}{2} \mathrm{U}_{s} \Sigma_{s}^{-1} \mathbf{V}_{s}^{H} E\left(\mathrm{~N}^{H} \mathrm{U}_{o} \mathrm{U}_{o}^{H} \mathrm{~N}\right) \mathbf{V}_{s} \Sigma_{s}^{-1}=-\frac{1}{2} \mathrm{U}_{s} \Sigma_{s}^{-2} \operatorname{Tr}\left(\mathrm{U}_{o} \mathrm{U}_{o}^{H}\right) \sigma_{n}^{2}
\end{gathered}
$$

\section{III.3 SUMMARY}

This chapter presents the analysis of subspace perturbations obtained from a singular value decomposition of a noisy data matrix. The expressions for the subspace perturbations are derived using the perturbation theory. We first introduce the general expression for perturbed subspace. Then the first-order subspace perturbations are reviewed followed by the derivation for the second-order subspace perturbations. Important statistics results of the subspace perturbations are discussed at the end of the chapter. The analysis of the subspace perturbations will provide the common ground for the comparison of the various subspace processing algorithms. 


\section{CHAPTER IV}

\section{BIAS ANALYSIS}

\section{IV.1 INTRODUCTION}

As mentioned in Chapter 1, performance analysis of DOA estimation bias is important yet challenging due to the theoretical difficulty and mathematical complexity involved. Early work on bias analysis has one or more the following limitations: (1) The asymptotic assumption that unbounded data sets are available may not be realistic in many array processing applications. (2) The inclusion of singular values and singular vectors in bias expressions which are obtained from nonlinear transformation, namely SVD, of data prevents us from observing the relationship between the estimated DOAs and physical parameters, such as source separation, signal coherence, numbers of serors and snapshots on estimation bias.

In this chapter, bias performance analysis for extrema-search approach, polynomial rooting approach and matrix-shifting approach are developed based on the common model underlying each approach. The ultimate goal is to express DOA estimation bias in term of fundamental parameters such as array manifold, source covariance and number of snapshots.

\section{IV.2 BIAS ANALYSIS FOR EXTREMA-SEARCH ALGORITHMS}

Extrema searching algorithms obtain DOAs through searching for minima in null spectrum (see Figure 3). A common model for the null spectrum function associated with 
MUSIC and Min-Norm searching algorithms is written as [8]

$$
P\left(\theta, \mathbf{U}_{o}\right)=\mathbf{a}^{H}(\theta) \mathbf{U}_{o} \mathbf{W} \mathbf{U}_{o}^{H} \mathbf{a}(\theta)
$$

where the weighting matrix $\mathbf{W}$ is specified as $\mathbf{I}$ for MUSIC and $\mathbf{W}=\overline{\mathbf{c}} \overline{\mathbf{c}}^{H}$ for Min-Norm, and $\theta$ is a scalar variable which represents a possible direction of arrival. For Min-Norm, $\overline{\mathbf{c}}=\mathbf{c} /\|\mathbf{c}\|^{2}$ where $\mathbf{c}^{H}$ is the first row of $\mathbf{U}_{o}$. From the orthogonality $\mathbf{a}^{H}\left(\theta_{k}\right) \mathbf{U}_{o}=0$, the noise-free null-spectrum satisfies

$$
P\left(\theta_{k}, \mathrm{U}_{o}\right)=0 \quad k=1, \cdots, K
$$

where $\theta_{k}$ is the $k$-th direction of arrival. In practice, with noise perturbation, null spectrum is no longer zero which results in perturbation in DOAs. DOA estimates for MUSIC and Min-Norm searching algorithms are obtained by

$$
\tilde{\theta}_{k}=\arg \min _{\theta_{k} \in \Theta} P\left(\tilde{\theta}_{k}, \tilde{\mathrm{U}}_{o}\right)
$$

where

$$
\tilde{\theta}_{k}=\theta_{k}+\Delta \theta_{k}
$$

$\Delta \theta_{k}$ is the estimation error of $k$-th DOA.

From (73), it can be seen that $P\left(\tilde{\theta}_{k}, \tilde{U}_{o}\right)$ satisfies

$$
\frac{\partial P\left(\tilde{\theta}_{k}, \tilde{U}_{o}\right)}{\partial \theta}=0
$$

A second-order expression for $E\left(\Delta \theta_{k}\right)$ which is accurate for SNR down to threshold region can be attained through two steps.

Step 1. Approximate $\Delta \theta_{k}$ by expanding $\frac{\partial P\left(\bar{\theta}_{k}, \overline{\mathbf{U}}_{o}\right)}{\partial \theta}$ to the second-order using Taylor series as

$$
\frac{\partial P\left(\tilde{\theta}_{k}, \tilde{\mathbf{U}}_{o}\right)}{\partial \theta}=\frac{\partial P\left(\theta_{k}, \tilde{\mathrm{U}}_{o}\right)}{\partial \theta}+\frac{\partial^{2} P\left(\theta_{k}, \tilde{\mathrm{U}}_{o}\right)}{\partial \theta^{2}} \Delta \theta_{k}+\frac{\partial^{3} P\left(\theta_{k}, \tilde{\mathrm{U}}_{o}\right)}{\partial \theta^{3}} \Delta^{2} \theta_{k} / 2
$$


Using (74), (75) is reduced to

$$
\frac{\partial P\left(\theta_{k}, \tilde{\mathbf{U}}_{o}\right)}{\partial \theta}+\frac{\partial^{2} P\left(\theta_{k}, \tilde{\mathbf{U}}_{o}\right)}{\partial \theta^{2}} \Delta \theta_{k}+\frac{\partial^{3} P\left(\theta_{k}, \tilde{\mathbf{U}}_{o}\right)}{\partial \theta^{3}} \Delta^{2} \theta_{k} / 2=0 .
$$

Let

$$
\begin{array}{llll}
\frac{\partial P\left(\theta_{k}, \mathbf{U}_{o}\right)}{\partial \theta} \stackrel{\text { def }}{=} N & \frac{\partial P\left(\theta_{k}, \tilde{\mathbf{U}}_{o}\right)}{\partial \theta} \stackrel{\text { def }}{=} N+\Delta N \\
\frac{\partial^{2} P\left(\theta_{k}, \mathbf{U}_{o}\right)}{\partial \theta^{2}} \stackrel{\text { def }}{=} D & \frac{\partial^{2} P\left(\theta_{k}, \tilde{\mathbf{U}}_{o}\right)}{\partial \theta^{2}} \stackrel{\text { def }}{=} D+\Delta D \\
\frac{\partial^{3} P\left(\theta_{k}, \mathbf{U}_{o}\right)}{\partial \theta^{3}} \stackrel{\text { def }}{=} & B & \frac{\partial^{3} P\left(\theta_{k}, \tilde{\mathbf{U}}_{o}\right)}{\partial \theta^{3}} \stackrel{\text { def }}{=} B+\Delta B .
\end{array}
$$

Then (76) can be written as

$$
(N+\Delta N)+(D+\Delta D) \Delta \theta_{k}+(B+\Delta B) \Delta^{2} \theta_{k} / 2=0 .
$$

Keep the terms up to the second-order

$$
N+\Delta N_{2}+D \Delta \theta_{k 2}+\Delta D_{1} \Delta \theta_{k 1}+B \Delta^{2} \theta_{k 1} / 2 \stackrel{2}{=} 0 .
$$

where $\Delta \theta_{k_{1}}=-\frac{\Delta N_{1}}{D}[8]$. The second-order approximation of $\Delta \theta_{k}$ is therefore expressed as

$$
\Delta \theta_{k 2}=-\frac{N+\Delta N_{2}}{D}+\frac{\Delta N_{1} \Delta D_{1}}{D^{2}}-\frac{B}{2 D} \Delta^{2} \theta_{k 1}
$$

Step 2. Take expectation on both sides to obtain general bias expression which is applicable to any searching algorithm

$$
E\left(\Delta \theta_{k 2}\right)=-E\left(\frac{N+\Delta N_{2}}{D}\right)+E\left(\frac{\Delta N_{1} \Delta D_{1}}{D^{2}}\right)-\frac{B}{2 D} E\left(\Delta^{2} \theta_{k 1}\right)
$$

\section{IV.2.1 Bias for MUSIC Searching Algorithm}

For MUSIC, the specific terms in (81) are given as

$$
N=0
$$




$$
\begin{aligned}
& D=2\left\|\mathbf{a}^{(1)}\left(\theta_{k}\right)^{H} \Omega_{o}\right\|^{2} \\
& B=6 \Re\left\{\mathbf{a}^{(1)}\left(\theta_{k}\right)^{H} \boldsymbol{\Omega}_{o} \mathbf{a}^{(2)}\left(\theta_{k}\right)\right\} \\
& E\left(\Delta N_{2}\right)=2(L-K) \sigma_{n}^{2} \Re\left\{\mathbf{a}^{(1)}\left(\theta_{k}\right)^{H}\left(\mathbf{A}^{\dagger}\right)^{H} \hat{\mathbf{R}}_{s}^{-1} \mathbf{e}_{k}\right\} \\
& E\left(\Delta N_{1} \Delta D_{1}\right)=2 \hat{\mathbf{R}}_{s}^{-1}(k, k) \sigma_{n}^{2} \Re\left\{\mathbf{a}^{(1)}\left(\theta_{k}\right)^{H} \boldsymbol{\Omega}_{o} \mathbf{a}^{(2)}\left(\theta_{k}\right)\right\} \\
& +4 \sigma_{n}^{2}\left\|\mathbf{a}^{(1)}\left(\theta_{k}\right)^{H} \boldsymbol{\Omega}_{o}\right\|^{2} \Re\left\{\mathbf{e}_{k} \hat{\mathbf{R}}_{s}^{-1} \mathbf{A}^{\dagger} \mathbf{a}^{(1)}\left(\theta_{k}\right)\right\} .
\end{aligned}
$$

Details of the derivation are referred to Appendix B.1. Substitute (82)-(86) into (81) and use the fact $\hat{\mathbf{R}}_{s}^{-1}=\frac{1}{M} \mathbf{R}_{s}^{-1}$ (see [23]) which gives

$$
\begin{aligned}
E\left(\Delta \theta_{k 2}\right)= & -\frac{\sigma_{n}^{2}(L-K-1)}{M\left\|\mathbf{a}^{(1)}\left(\theta_{k}\right)^{H} \Omega_{o}\right\|^{2}} \Re\left\{\mathbf{a}^{(1)}\left(\theta_{k}\right)^{H}\left(\mathbf{A}^{\dagger}\right)^{H} \mathbf{R}_{s}^{-1} \mathbf{e}_{k}\right\} \\
& -\frac{\Re\left\{\mathbf{a}^{(1)}\left(\theta_{k}\right)^{H} \Omega_{o} \mathbf{a}^{(2)}\left(\theta_{k}\right)\right\}}{2\left\|\mathbf{a}^{(1)}\left(\theta_{k}\right)^{H} \Omega_{o}\right\|^{2}} E\left(\Delta \theta_{k 1}^{2}\right) .
\end{aligned}
$$

IV.2.2 Bias for Min-Norm Searching Algorithm

For Min-Norm, the specific terms in (81) are given as

$$
\begin{aligned}
N= & 0 \\
D= & \frac{2}{\left\|\mathbf{e}_{1}^{H} \boldsymbol{\Omega}_{o}\right\|^{4}}\left|\mathbf{a}^{(1)}\left(\theta_{k}\right)^{H} \boldsymbol{\Omega}_{o} \mathbf{e}_{1}\right|^{2} \\
B= & \frac{6}{\left\|\mathbf{e}_{1}^{H} \boldsymbol{\Omega}_{o}\right\|^{4}} \Re\left\{\mathbf{a}^{(1)}\left(\theta_{k}\right)^{H} \boldsymbol{\Omega}_{o} \mathbf{e}_{1} \mathbf{e}_{1}^{H} \boldsymbol{\Omega}_{o} \mathbf{a}^{(2)}\left(\theta_{k}\right)\right\} \\
E\left(\Delta N_{2}\right)= & \frac{2(L-K) \sigma_{n}^{2}}{\left\|\mathbf{e}_{1}^{H} \boldsymbol{\Omega}_{o}\right\|^{4}} \Re\left\{\mathbf{a}^{(1)}\left(\theta_{k}\right)^{H} \boldsymbol{\Omega}_{o} \mathbf{e}_{1} \mathbf{e}_{1}^{H}\left(\mathbf{A}^{\dagger}\right)^{H} \hat{\mathbf{R}}_{s}^{-1} \mathbf{e}_{k}\right\} \\
& +\frac{2 \sigma_{n}^{2}}{\left\|\mathbf{e}_{1}^{H} \boldsymbol{\Omega}_{o}\right\|^{2}} \Re\left\{\mathbf{a}^{(1)}\left(\theta_{k}\right)^{H}\left(\mathbf{A}^{\dagger}\right)^{H} \hat{\mathbf{R}}_{s}^{-1} \mathbf{e}_{k}\right\} \\
E\left(\Delta N_{1} \Delta D_{1}\right)= & \frac{4 \sigma_{n}^{2}\left|\mathbf{a}^{(1)}\left(\theta_{k}\right)^{H} \boldsymbol{\Omega}_{o} \mathbf{e}_{1}\right|^{2}}{\left\|\mathbf{e}_{1}^{H} \boldsymbol{\Omega}_{o}\right\|^{8}} \Re\left\{\mathbf{a}^{(1)}\left(\theta_{k}\right)^{H} \boldsymbol{\Omega}_{o} \mathbf{e}_{1} \mathbf{e}_{1}^{H}\left(\mathbf{A}^{\dagger}\right)^{H} \hat{\mathbf{R}}_{s}^{-1} \mathbf{e}_{k}\right\} \\
& +\frac{4 \sigma_{n}^{2}\left|\mathbf{a}^{(1)}\left(\theta_{k}\right)^{H} \boldsymbol{\Omega}_{o} \mathbf{e}_{1}\right|^{2}}{\left\|\mathbf{e}_{1}^{H} \boldsymbol{\Omega}_{o}\right\|^{6}} \Re\left\{\mathbf{a}^{(1)}\left(\theta_{k}\right)^{H}\left(\mathbf{A}^{\dagger}\right)^{H} \hat{\mathbf{R}}_{s}^{-1} \mathbf{e}_{k}\right\} \\
& +\frac{2 \sigma_{n}^{2} \hat{\mathbf{R}}_{s}^{-1}(k, k)}{\left\|\mathbf{e}_{1}^{H} \boldsymbol{\Omega}_{o}\right\|^{6}} \Re\left\{\mathbf{a}^{(1)}\left(\theta_{k}\right)^{H} \boldsymbol{\Omega}_{o} \mathbf{e}_{1} \mathbf{e}_{1}^{H} \Omega_{o} \mathbf{a}^{(2)}\left(\theta_{k}\right)\right\} .
\end{aligned}
$$


Detailed derivation is referred to Appendix B.2. Substitute (88)-(92) into (81) and use the fact $\hat{\mathbf{R}}_{s}^{-1}=\frac{1}{M} \mathbf{R}_{s}^{-1}$ which gives

$$
\begin{aligned}
E\left(\Delta \theta_{k 2}\right)= & -\frac{\sigma_{n}^{2}(L-K-1)}{M\left|\mathbf{a}^{(1)}\left(\theta_{k}\right)^{H} \Omega_{o} \mathbf{e}_{1}\right|^{2}} \Re\left\{\mathbf{a}^{(1)}\left(\theta_{k}\right)^{H} \Omega_{o} \mathbf{e}_{1} \mathbf{e}_{1}^{H}\left(\mathbf{A}^{\dagger}\right)^{H} \mathbf{R}_{s}^{-1} \mathbf{e}_{k}\right\} \\
& -\frac{\Re\left\{\mathbf{a}^{(1)}\left(\theta_{k}\right)^{H} \Omega_{o} \mathbf{e}_{1} \mathbf{e}_{1}^{H} \boldsymbol{\Omega}_{o} \mathbf{a}^{(2)}\left(\theta_{k}\right)\right\}}{2 M\left|\mathbf{a}^{(1)}\left(\theta_{k}\right)^{H} \Omega_{o} \mathbf{e}_{1}\right|^{2}} E\left(\Delta \theta_{k 1}^{2}\right) .
\end{aligned}
$$

\section{IV.3 BIAS ANALYSIS OF POLYNOMIAL ROOTING ALGORITHMS}

DOAs in rooting methods are determined from the roots of spectral polynomial. A common spectral polynomial for Min-Norm and Root-MUSIC (also Pisarenko's method) can be written as [8]

$$
P(z)=\mathbf{a}\left(z^{-1}\right)^{T} \mathbf{U}_{o} \mathbf{W} \mathbf{U}_{o}^{H} \mathbf{a}(z)=A \prod_{i=1}^{L-1}\left(1-r_{i} z^{-1}\right)\left(1-r_{i}^{*} z\right),
$$

where the weighting matrix $\mathbf{W}$ is the same as in extrema-search algorithm and $A$ is a scaling factor. The relationship between the roots of the spectral polynomial and the directions of arrival is

$$
r_{i}=e^{j \frac{2 \pi d}{\lambda_{c}} \sin \theta_{i}}
$$

The difference between searching algorithms and rooting algorithms is that searching algorithms search for minima of a function in one dimension while rooting algorithms search for global minima over multi-dimensional space to obtain roots simultaneously, thus when two DOAs are closely spaced, rooting method may be able to give true DOAs while searching algorithm may have only one extremum resulting in the failure of resolving two DOAs near threshold. If two roots are well separated, rooting approach intuitively should have the same performance as searching approach, as we will show in the next section. 
The spectral polynomial at the signal root locations are minima since $P(z)$ is nonnegative. For noise-free spectral polynomial, minima are equal to zero and roots are on the unit circle. When the spectral polynomial is perturbed, minima are no longer zero and signal roots are slightly off unit circle (refer to Figure 4 and Figure 5). The local minima of either noise-free spectral polynomial or perturbed spectral polynomial are obtained by setting the first derivative of spectral polynomial to zero. For perturbed spectral polynomial, i.e.

$$
\frac{\partial P\left(\tilde{r}_{k}, \tilde{\mathbf{U}}_{o}\right)}{\partial z}=0 \quad k=1, \cdots, K
$$

where

$$
\tilde{r}_{k}=r_{k}+\Delta r_{k}
$$

$\Delta r_{k}$ is the perturbation of the true root which induces error in DOA estimation.

A derivation for the second-order $E\left(\Delta \theta_{k}\right)$ which is valid over a wide range of SNR extending down to the threshold region is divided into three steps.

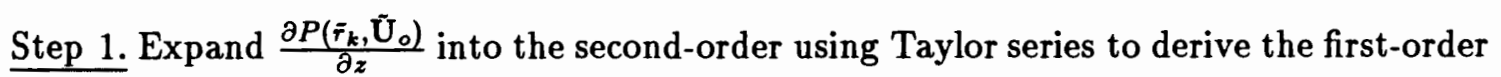
and the second-order expressions of $\Delta r_{k}$,

$$
\frac{\partial P\left(\tilde{r}_{k}, \tilde{\mathbf{U}}_{o}\right)}{\partial z}=\frac{\partial P\left(r_{k}, \tilde{\mathrm{U}}_{o}\right)}{\partial z}+\frac{\partial^{2} P\left(r_{k}, \tilde{\mathbf{U}}_{o}\right)}{\partial z^{2}} \Delta r_{k}+\frac{\partial^{3} P\left(r_{k}, \tilde{\mathbf{U}}_{o}\right)}{\partial z^{3}} \Delta^{2} r_{k} / 2 .
$$

Using (96), (98) is reduced to

$$
\frac{\partial P\left(r_{k}, \tilde{\mathbf{U}}_{o}\right)}{\partial z}+\frac{\partial^{2} P\left(r_{k}, \tilde{\mathbf{U}}_{o}\right)}{\partial z^{2}} \Delta r_{k}+\frac{\partial^{3} P\left(r_{k}, \tilde{\mathbf{U}}_{o}\right)}{\partial z^{3}} \Delta^{2} r_{k} / 2=0
$$

Let

$$
\begin{array}{ccll}
\frac{\partial P\left(r_{k}, \mathrm{U}_{o}\right)}{\partial z} \stackrel{\text { def }}{=} N & \frac{\partial P\left(r_{k}, \tilde{\mathrm{U}}_{o}\right)}{\partial z} \stackrel{\text { def }}{=} N+\Delta N \\
\frac{\partial^{2} P\left(r_{k}, \mathrm{U}_{o}\right)}{\partial z^{2}} \stackrel{\text { def }}{=} D & \frac{\partial^{2} P\left(r_{k}, \tilde{\mathrm{U}}_{o}\right)}{\partial z^{2}} \stackrel{\text { def }}{=} D+\Delta D \\
\frac{\partial^{3} P\left(r_{k}, \mathrm{U}_{o}\right)}{\partial z^{3}} \stackrel{\text { def }}{=} B & \frac{\partial^{3} P\left(r_{k}, \tilde{\mathrm{U}}_{o}\right)}{\partial z^{3}} \stackrel{\text { def }}{=} B+\Delta B .
\end{array}
$$


Then (99) can be written as

$$
(N+\Delta N)+(D+\Delta D) \Delta r_{k}+(B+\Delta B) \Delta^{2} r_{k} / 2=0
$$

Keep the equation up to second-order as

$$
N+\Delta N_{2}+D \Delta r_{k 2}+\Delta D_{1} \Delta r_{k 1}+B \Delta^{2} r_{k 1} / 2 \stackrel{2}{=} 0
$$

where $\Delta r_{k 1}$ can be obtained by keeping (101) to first-order as

$$
\Delta r_{k_{1}}=-\frac{\left(N+\Delta N_{1}\right)}{D}
$$

Substitute (103) into (102) to obtain $\Delta r_{k 2}$

$$
\Delta r_{k 2}=-\frac{\left(N+\Delta N_{2}\right)}{D}+\frac{\left(\Delta D_{1} N+\Delta D_{1} \Delta N_{1}\right)}{D^{2}}-\frac{B\left(N+\Delta N_{1}\right)^{2}}{2 D^{3}}
$$

Step 2. A second-order approximation of $\Delta \theta_{k}$ can be derived using the angle-root relation in Appendix D.2 as

$$
\Delta \theta_{k 2}=C_{k} \Im\left(\frac{\Delta r_{k 2}}{r_{k}}-\frac{\Delta^{2} r_{k 1}}{2 r_{k}^{2}}\right)+\frac{\tan \theta_{k}}{2} \Delta^{2} \theta_{k 1}
$$

Substitute (103) and (104) into (105)

$$
\begin{aligned}
\Delta \theta_{k 2}= & \frac{C_{k}}{r_{k}} \Im\left[-\frac{\left(N+\Delta N_{2}\right)}{D}+\frac{\left(N \Delta D_{1}+\Delta D_{1} \Delta N_{1}\right)}{D^{2}}-\frac{B\left(N+\Delta N_{1}\right)^{2}}{2 D^{3}}\right] \\
& -\frac{C_{k}}{2 r_{k}^{2}} \Im\left[\frac{\left(N+\Delta N_{1}\right)^{2}}{D^{2}}\right]+\frac{\tan \theta_{k}}{2} \Delta^{2} \theta_{k 1} .
\end{aligned}
$$

Step 3. Take expectation on both sides of equation to obtain general bias expression for any polynomial rooting algorithms

$$
\begin{aligned}
E\left(\Delta \theta_{k 2}\right)= & \frac{C_{k}}{r_{k}} \Im\left\{E\left[-\frac{\left(N+\Delta N_{2}\right)}{D}+\frac{\left(N \Delta D_{1}+\Delta D_{1} \Delta N_{1}\right)}{D^{2}}-\frac{B\left(N+\Delta N_{1}\right)^{2}}{2 D^{3}}\right]\right\} \\
& -\frac{C_{k}}{2 r_{k}^{2}} \Im\left\{E\left[\frac{\left(N+\Delta N_{1}\right)^{2}}{D^{2}}\right]\right\}+\frac{\tan \theta_{k}}{2} E\left(\Delta^{2} \theta_{k 1}\right)
\end{aligned}
$$




\section{IV.3.1 Bias for Root MUSIC}

For Root MUSIC, the specific terms in (106) are given as

$$
\begin{aligned}
N= & 0 \\
D= & -2 r_{k}^{-2}\left\|\mathbf{a}^{(1)}\left(r_{k}^{-1}\right)^{T} \Omega_{o}\right\|^{2} \\
B= & 6 r_{k}^{-3}\left\|\mathbf{a}^{(1)}\left(r_{k}^{-1}\right)^{T} \Omega_{o}\right\|^{2}+6 j r_{k}^{-3} \Im\left\{r_{k}^{-1} \mathbf{a}^{(2)}\left(r_{k}^{-1}\right)^{T} \Omega_{o} \mathbf{a}^{(1)}\left(r_{k}\right)\right\} \\
E\left(\Delta N_{2}\right)= & -2 j(L-K) \sigma_{n}^{2} r_{k}^{-1} \Im\left\{r_{k}^{-1} \mathbf{a}^{(1)}\left(r_{k}^{-1}\right)^{T}\left(\mathbf{A}^{\dagger}\right)^{H} \hat{\mathbf{R}}_{s}^{-1} \mathbf{e}_{k}\right\} \\
E\left(\Delta^{2} N_{1}\right)= & -2 \sigma_{n}^{2} r_{k}^{-2}\left\|\mathbf{a}^{(1)}\left(r_{k}^{-1}\right)^{T} \Omega_{o}\right\|^{2} \hat{\mathbf{R}}_{s}^{-1}(k, k) \\
E\left(\Delta N_{1} \Delta D_{1}\right)= & 4 j \sigma_{n}^{2} r_{k}^{-3}\left\|\mathbf{a}^{(1)}\left(r_{k}^{-1}\right)^{T} \Omega_{o}\right\|^{2} \Im\left\{r_{k}^{-1} \mathbf{a}^{(1)}\left(r_{k}^{-1}\right)^{T}\left(\mathbf{A}^{\dagger}\right)^{H} \hat{\mathbf{R}}_{s}^{-1} \mathbf{e}_{k}\right\} \\
& +2 j \sigma_{n}^{2} r_{k}^{-3} \hat{\mathbf{R}}_{s}^{-1}(k, k) \Im\left\{r_{k}^{-1} \mathbf{a}^{(2)}\left(r_{k}^{-1}\right)^{T} \Omega_{o} \mathbf{a}^{(1)}\left(r_{k}\right)\right\} \\
& +2 \sigma_{n}^{2} r_{k}^{-3} \hat{\mathbf{R}}_{s}^{-1}(k, k)\left\|\mathbf{a}^{(1)}\left(r_{k}^{-1}\right)^{T} \Omega_{o}\right\|^{2} .
\end{aligned}
$$

For detail derivation of (107)-(112), see Appendix C.1. Substitute (107)-(112)into (106) and use the fact $\hat{\mathbf{R}}_{s}^{-1}=\frac{1}{M} \mathbf{R}_{s}^{-1}$ to gives an explicit bias expression

$$
\begin{aligned}
E\left(\Delta \theta_{k 2}\right)= & -\frac{C_{k}(L-K-1) \sigma_{n}^{2}}{M\left\|\mathbf{a}^{(1)}\left(r_{k}^{-1}\right)^{T} \mathbf{\Omega}_{o}\right\|^{2}} \Im\left\{r_{k}^{-1} \mathbf{a}^{(1)}\left(r_{k}^{-1}\right)^{T}\left(\mathbf{A}^{\dagger}\right)^{H} \mathbf{R}_{s}^{-1} \mathbf{e}_{k}\right\} \\
& -\frac{C_{k} \mathbf{R}_{s}^{-1}(k, k) \sigma_{n}^{2}}{4 M\left\|\mathbf{a}^{(1)}\left(r_{k}^{-1}\right)^{T} \Omega_{o}\right\|^{4}} \Im\left\{r_{k}^{-1} \mathbf{a}^{(2)}\left(r_{k}^{-1}\right)^{T} \Omega_{o} \mathbf{a}^{(1)}\left(r_{k}\right)\right\} \\
& +\frac{\tan \theta_{k}}{2} E\left(\Delta \theta_{k 1}^{2}\right) .
\end{aligned}
$$

\section{IV.3.2 Bias for Root Min-Norm}

For Root Min-Norm, the specific terms are given as

$$
\begin{aligned}
N & =0 \\
D & =\frac{-2 r_{k}^{-2}}{\left\|\mathbf{e}_{1}^{H} \Omega_{o}\right\|^{4}}\left|\mathbf{a}^{(1)}\left(r_{k}^{-1}\right)^{T} \Omega_{o} \mathbf{e}_{1}\right|^{2}
\end{aligned}
$$




$$
\begin{aligned}
& B=\frac{1}{\left\|\mathbf{e}_{1}^{H} \Omega_{o}\right\|^{4}}\left\{6 r_{k}^{-3}\left|\mathbf{a}^{(1)}\left(r_{k}^{-1}\right)^{T} \Omega_{o} \mathbf{e}_{1}\right|^{2}\right. \\
& \left.+6 j r_{k}^{-3} \Im\left[r_{k}^{-1} \mathbf{a}^{(2)}\left(r_{k}^{-1}\right)^{T} \Omega_{o} \mathbf{e}_{1} \mathbf{e}_{1}^{H} \boldsymbol{\Omega}_{o} \mathbf{a}^{(1)}\left(r_{k}\right)\right]\right\} \\
& E\left(\Delta N_{2}\right)=-\frac{2 j(L-K) r_{k}^{-1} \sigma_{n}^{2}}{\left\|\mathbf{e}_{1}^{H} \Omega_{o}\right\|^{4}} \Im\left\{r_{k}^{-1} \mathbf{a}^{(1)}\left(r_{k}^{-1}\right)^{T} \Omega_{o} \mathbf{e}_{1} \mathbf{e}_{1}^{H}\left(\mathbf{A}^{\dagger}\right)^{H} \hat{\mathbf{R}}_{s}^{-1} \mathbf{e}_{k}\right. \\
& \left.+\left\|\mathbf{e}_{1}^{H} \Omega_{o}\right\|^{2} r_{k}^{-1} \mathbf{a}^{(1)}\left(r_{k}^{-1}\right)^{T}\left(\mathbf{A}^{\dagger}\right)^{H} \hat{\mathbf{R}}_{s}^{-1} \mathbf{e}_{k}\right\} \\
& E\left(\Delta^{2} N_{1}\right)=-\frac{2 \sigma_{n}^{2} r_{k}^{-2}}{\left\|\mathbf{e}_{1}^{H} \Omega_{o}\right\|^{6}}\left|\mathbf{a}^{(1)}\left(r_{k}^{-1}\right)^{T} \Omega_{\circ} \mathbf{e}_{1}\right|^{2} \hat{\mathbf{R}}_{s}^{-1}(k, k) \\
& E\left(\Delta N_{1} \Delta D_{1}\right)=\frac{\sigma_{n}^{2}}{\left\|\mathbf{e}_{1}^{H} \Omega_{o}\right\|^{8}}\left\{2 r_{k}^{-3} \hat{\mathbf{R}}_{s}^{-1}(k, k)\left\|\mathbf{e}_{1}^{H} \Omega_{o}\right\|^{2}\left|\mathbf{a}^{(1)}\left(r_{k}^{-1}\right)^{T} \Omega_{o} \mathbf{e}_{1}\right|^{2}\right. \\
& +4 j r_{k}^{-3}\left\|\mathbf{e}_{1}^{H} \Omega_{o}\right\|^{2}\left|\mathbf{a}^{(1)}\left(r_{k}^{-1}\right)^{T} \Omega_{o} \mathbf{e}_{1}\right|^{2} \Im\left\{r_{k}^{-1} \mathbf{a}^{(1)}\left(r_{k}^{-1}\right)^{T}\left(\mathbf{A}^{\dagger}\right)^{H} \hat{\mathbf{R}}_{s}^{-1} \mathbf{e}_{k}\right\} \\
& +4 j r_{k}^{-3}\left|\mathbf{a}^{(1)}\left(r_{k}^{-1}\right)^{T} \Omega_{o} \mathbf{e}_{1}\right|^{2} \Im\left\{r_{k}^{-1} \mathbf{a}^{(1)}\left(r_{k}^{-1}\right)^{T} \Omega_{o} \mathbf{e}_{1} \mathbf{e}_{1}^{H}\left(\mathbf{A}^{\dagger}\right)^{H} \hat{\mathbf{R}}_{s}^{-1} \mathbf{e}_{k}\right\} \\
& \left.+2 j r_{k}^{-3}\left\|\mathbf{e}_{1}^{H} \boldsymbol{\Omega}_{o}\right\|^{2} \Im\left\{r_{k}^{-1} \hat{\mathbf{R}}_{s}^{-1}(k, k) \mathbf{a}^{(2)}\left(r_{k}^{-1}\right)^{T} \boldsymbol{\Omega}_{o} \mathbf{e}_{1} \mathbf{e}_{1}^{H} \boldsymbol{\Omega}_{o} \mathbf{a}^{(1)}\left(r_{k}\right)\right\}\right\} .
\end{aligned}
$$

For detailed derivation of (113)-(118), see Appendix C.2. Substitute (113)-(118) into (106) and use the fact $\hat{\mathbf{R}}_{s}^{-1}=\frac{1}{M} \mathbf{R}_{s}^{-1}$ to obtain an concise bias espression

$$
\begin{aligned}
E\left(\Delta \theta_{k 2}\right)= & -\frac{C_{k} \sigma_{n}^{2}(L-K-1)}{M\left|\mathbf{a}^{(1)}\left(r_{k}^{-1}\right)^{T} \Omega_{o} \mathbf{e}_{1}\right|^{2}} \Im\left\{r_{k}^{-1} \mathbf{a}^{(1)}\left(r_{k}^{-1}\right)^{T} \Omega_{o} \mathbf{e}_{1} \mathbf{e}_{1}^{H}\left(\mathbf{A}^{\dagger}\right)^{H} \mathbf{R}_{s}^{-1} \mathbf{e}_{k}\right\} \\
& -\frac{C_{k} \sigma_{n}^{2} \mathbf{R}_{s}^{-1}(k, k)\left\|\mathbf{e}_{1}^{H} \Omega_{o}\right\|^{2}}{4 M\left|\mathbf{a}^{(1)}\left(r_{k}^{-1}\right)^{T} \Omega_{o} \mathbf{e}_{1}\right|^{2}} \Im\left\{r_{k}^{-1} \mathbf{a}^{(2)}\left(r_{k}^{-1}\right)^{T} \Omega_{o} \mathbf{e}_{1} \mathbf{e}_{1}^{H} \Omega_{o} \mathbf{a}^{(1)}\left(r_{k}\right)\right\} \\
& +\frac{\tan \theta_{k}}{2} E\left(\Delta \theta_{k 1}^{2}\right)
\end{aligned}
$$

\section{IV.4 EQUATING OF EXTREMA SEARCHING ALGORITHMS AND}

\section{POLYNOMIAL ROOTING ALGORITHMS}

As mentioned early, Provided that a uniform line array is used, polynomial rooting algorithms have the same performance as extrema searching algorithms if the two DOAs are well separated. In the following, we will prove this by equating rooting algorithms and searching algorithms through the relation (95). 
Using (95), it can be shown

$$
\begin{aligned}
\mathbf{a}^{(1)}\left(\theta_{k}\right) & =j \frac{2 \pi d \cos \theta_{k}}{\lambda_{c}} r_{k} \mathbf{a}^{(1)}\left(r_{k}\right) \\
& =\frac{j r_{k} \mathbf{a}^{(1)}\left(r_{k}\right)}{C_{k}} \\
\mathbf{a}^{(2)}\left(\theta_{k}\right) & =-j \frac{2 \pi d \sin \theta_{k}}{\lambda_{c}} r_{k} \mathbf{a}^{(1)}\left(r_{k}\right)-\left(\frac{2 \pi d \cos \theta_{k}}{\lambda_{c}}\right)^{2} r_{k} \mathbf{a}^{(1)}\left(r_{k}\right)-\left(\frac{2 \pi d \cos \theta_{k}}{\lambda_{c}}\right)^{2} r_{k}^{2} \mathbf{a}^{(2)}\left(r_{k}\right) \\
& =-\frac{j \tan \theta_{k} r_{k} \mathbf{a}^{(1)}\left(r_{k}\right)}{C_{k}}-\frac{r_{k} \mathbf{a}^{(1)}\left(r_{k}\right)}{C_{k}^{2}}-\frac{r_{k}^{2} \mathbf{a}^{(2)}\left(r_{k}\right)}{C_{k}^{2}} .
\end{aligned}
$$

Now substitute (119) and (120) into (87) and refer $E\left(\Delta \theta_{k}^{2}\right)$ to Table 1.

$$
\begin{aligned}
& E\left(\Delta \theta_{k 2}\right) \\
& =-\frac{C_{k}^{4} \mathbf{R}_{s}^{-1}(k, k) \sigma_{n}^{2}}{4 M\left\|\mathbf{a}^{(1)}\left(r_{k}^{-1}\right)^{T} \Omega_{o}\right\|^{4}} \Re\left\{-\frac{\tan \theta_{k}}{C_{k}^{2}} \mathbf{a}^{(1)}\left(r_{k}^{-1}\right)^{T} \Omega_{o} \mathbf{a}^{(1)}\left(r_{k}\right)+\frac{j}{C_{k}^{3}} \mathbf{a}^{(1)}\left(r_{k}^{-1}\right)^{T} \Omega_{o} \mathbf{a}^{(1)}\left(r_{k}\right)\right. \\
& \left.+\frac{j r_{k}}{C_{k}^{3}} \mathbf{a}^{(1)}\left(r_{k}^{-1}\right)^{T} \Omega_{o} \mathbf{a}^{(2)}\left(r_{k}\right)\right\}+\frac{C_{k} \sigma_{n}^{2}(L-P-1)}{M\left\|\mathbf{a}^{(1)}\left(r_{k}^{-1}\right)^{T} \Omega_{o}\right\|^{2}} \Re\left\{j r_{k}^{-1} \mathbf{a}^{(1)}\left(r_{k}^{-1}\right)^{T}\left(\mathbf{A}^{\dagger}\right)^{H} \mathbf{R}_{s}^{-1} \mathbf{e}_{k}\right\} \\
& =-\frac{C_{k} \mathbf{R}_{s}^{-1}(k, k) \sigma_{n}^{2}}{4 M\left\|\mathbf{a}^{(1)}\left(r_{k}^{-1}\right)^{T} \Omega_{o}\right\|^{4}} \Re\left\{j r_{k} \mathbf{a}^{(1)}\left(r_{k}^{-1}\right)^{T} \Omega_{o} \mathbf{a}^{(2)}\left(r_{k}\right)\right\}+\frac{\tan \theta_{k} C_{k}^{2} \mathbf{R}_{s}^{-1}(k, k) \sigma_{n}^{2}}{4 M\left\|\mathbf{a}^{(1)}\left(r_{k}^{-1}\right)^{T} \Omega_{o}\right\|^{2}} \\
& +\frac{C_{k} \sigma_{n}^{2}(L-P-1)}{M\left\|\mathbf{a}^{(1)}\left(r_{k}^{-1}\right)^{T} \Omega_{o}\right\|^{2}} \Re\left\{j r_{k}^{-1} \mathbf{a}^{(1)}\left(r_{k}^{-1}\right)^{T}\left(\mathbf{A}^{\dagger}\right)^{H} \mathbf{R}_{s}^{-1} \mathbf{e}_{k}\right\} \text {. }
\end{aligned}
$$

Clearly, the bias for MUSIC and Root MUSIC are identical. Similarly, substitute (119) and (120) into (93) and use Table 1 to equate Min-Norm search algorithm with Min-Norm.

\section{TABLE I}

MSE FOR MUSIC, MIN-NORM AND ROOT MUSIC, ROOT MIN-NORM

\begin{tabular}{|c|c|c|c|c|}
\hline & MUSIC & Min-Norm & Root MUSIC & Root Min-Norm \\
\hline \hline$E\left(\Delta \theta_{k}^{2}\right)$ & $\frac{\sigma_{n}^{2} \mathbf{R}_{s}^{-1}(k, k)}{2 M\left\|\mathbf{a}^{(1)}\left(\theta_{k}\right)^{H} \Omega_{o}\right\|^{2}}$ & $\frac{\sigma_{n}^{2} R_{s}^{-1}(k, k)\left\|\left.\right|_{1} ^{H} \Omega_{o}\right\|^{2}}{2 M\left\|\mathbf{a}^{(1)}\left(\theta_{k}\right)^{H} \Omega_{o} \mathbf{e}_{1}\right\|^{2}}$ & $\frac{C_{k}^{2} \sigma_{n}^{2} \mathbf{R}_{s}^{-1}(k, k)}{2 M\left\|\mathbf{a}^{(1)}\left(r_{k}^{-1}\right)^{T} \Omega_{o}\right\|^{2}}$ & $\frac{C_{k}^{2} \sigma_{n}^{2} \bar{R}_{s}^{-1}(k, k)\left\|\left.\right|_{1} ^{\beta} \Omega_{o}\right\|^{2}}{2 M\left\|\mathbf{a}^{(1)}\left(r_{k}^{-1}\right)^{T} \Omega_{o} e_{1}\right\|^{2}}$ \\
\hline
\end{tabular}




\section{IV.5 BIAS ANALYSIS FOR MATRIX-SHIFTING ALGORITHMS}

As introduced in Chapter 2, ESPRIT, SSR and Matrix-Pencil method utilize the shift-invariant structure of the signal-subspace which again can be explored using array manifold

$$
\mathbf{A}^{\downarrow} \mathbf{D}=\mathbf{A}^{\dagger}
$$

where $D$ is a diagonal matrix with elements $\lambda_{k}=e^{j \frac{2 \pi d}{\lambda_{c}} \sin \theta_{k}}$. A common model for SSR and ESPRIT is rewritten as [8]

$$
\boldsymbol{\Phi}^{\downarrow} \mathbf{F}=\boldsymbol{\Phi}^{\uparrow}
$$

where

$$
\mathbf{\Phi}=\mathbf{U}_{\mathbf{s}} \mathbf{W}
$$

The weighting matrix $\mathbf{W}$ is specialized as $\mathbf{I}$ for ESPRIT and $\mathbf{W}=\boldsymbol{\Sigma}_{s}^{\frac{1}{2}}$ for SSR. Since $\mathbf{A}$ and $\mathbf{\Phi}$ span the same subspace, $\mathbf{D}$ and $\mathbf{F}$ are similar

$$
\mathbf{D}=\mathbf{L}^{-1} \mathbf{F L} \text {. }
$$

Matrix shifting algorithms estimate DOAs by determining the eigenvalues $\lambda_{k}$ of the matrix $\mathbf{F}$ which are the same as the diagonal elements of $\mathbf{D}$.

In Chapter 2, we have proved that SSR and ESPRIT are essentially the same when uniform line array or matched array senor doublets are used. In the following bias analysis, we only discuss ESPRIT in particular. When noise presents, eigenvalues is perturbed (see Figure 6). With $\mathbf{W}=\mathbf{I}$ for ESPRIT, (122) becomes

$$
\left(\mathbf{U}_{s}^{\downarrow}+\Delta \mathbf{U}_{s}^{\dagger}\right)(\mathbf{F}+\Delta \mathbf{F})=\mathbf{U}_{s}^{\uparrow}+\Delta \mathbf{U}_{s}^{\uparrow}
$$

where $\Delta \mathbf{F}$ is the perturbation matrix which induces error in DOA estimates. A derivation of second-order bias which is accurate for SNR down to threshold region 
is outlined below.

Step 1. Premultiply both sides of (125) with $\left(\mathbf{U}_{s}^{\downarrow}+\Delta \mathbf{U}_{s}^{!}\right)^{H}$, then expand it up to the second-order to derive second-order approximation of $\Delta \mathbf{F}$ as

$$
\begin{aligned}
& \mathrm{U}_{s}^{!^{H}} \mathbf{U}_{s}^{\downarrow} \Delta \mathbf{F}_{2}+\left(\Delta \mathbf{U}_{s 1}^{{ }^{H}} \mathbf{U}_{s}^{\downarrow}+\mathbf{U}_{s}^{\downarrow^{H}} \Delta \mathbf{U}_{s 1}^{\downarrow}\right) \Delta \mathbf{F}_{1} \\
& \stackrel{2}{=} \Delta \mathbf{U}_{s 1}^{\perp^{H}}\left(\Delta \mathbf{U}_{s 1}^{\uparrow}-\Delta \mathbf{U}_{s 1}^{\downarrow} \mathbf{F}\right)+\mathbf{U}_{s}^{l^{H}}\left(\Delta \mathbf{U}_{s 2}^{\uparrow}-\Delta \mathbf{U}_{s 2}^{\downarrow} \mathbf{F}\right) \text {. }
\end{aligned}
$$

Substitute $\Delta \mathbf{F}_{1}=\left(\mathbf{U}_{s}^{\downarrow}\right)^{\dagger}\left(\Delta \mathbf{U}_{s 1}^{\dagger}-\Delta \mathbf{U}_{s 1}^{\downarrow} \mathbf{F}\right)$ into the equation (126), we have

$$
\begin{aligned}
\Delta \mathbf{F}_{2}= & \mathbf{U}_{s}^{\dagger}\left(\Delta \mathbf{U}_{s 2}^{\uparrow}-\Delta \mathbf{U}_{s 2}^{\downarrow} \mathbf{F}\right)-\mathbf{U}_{s}^{\dagger} \Delta \mathbf{U}_{s 1}^{\downarrow} \mathbf{U}_{s}^{\dagger}\left(\Delta \mathbf{U}_{s 1}^{\dagger}-\Delta \mathbf{U}_{s 1}^{\downarrow} \mathbf{F}\right) \\
& +\left(\mathbf{U}_{s}^{!} \mathbf{U}_{s}^{\downarrow}\right)^{-1} \Delta \mathbf{U}_{s 1}^{!^{H}}\left(\mathbf{I}-\mathbf{U}_{s}^{\downarrow} \mathbf{U}_{s}^{\dagger}\right)\left(\Delta \mathbf{U}_{s 1}^{\dagger}-\Delta \mathbf{U}_{s 1}^{\downarrow} \mathbf{F}\right) .
\end{aligned}
$$

Step 2. Calculate the expectation of second-order perturbation of the eigenvalues of $\tilde{\mathbf{F}}$ due to $\Delta \mathbf{F}$.

$$
E\left(\Delta \lambda_{k 2}\right)=\frac{\sigma_{n}^{2}}{M}\left[-\lambda_{k}(L-K-1)-\operatorname{Tr}\left(\mathbf{P}_{\mathbf{A}\rfloor} \mathbf{I}^{\dagger} \mathbf{I}^{\downarrow^{H}}\right)\right] \mathbf{e}_{k}^{H}\left(\mathbf{A}^{\downarrow^{H}} \mathbf{A}^{\downarrow}\right)^{-1} \mathbf{R}_{s}^{-1} \mathbf{e}_{k}
$$

where $\mathbf{P}_{\mathbf{A} \downarrow}$ is the projection matrix of $\mathbf{A}^{\downarrow}$. The detailed derivation is in Appendix D.1.

Step 3. Obtain bias expression using angle-root relation in Appendix D.2. Take expectation on both sides of the equation (201).

$$
E\left(\Delta \theta_{k 2}\right)=C_{k} \Im\left\{E\left(\frac{\Delta r_{k 2}}{r_{k}}-\frac{\Delta^{2} r_{k 1}}{2 r_{k}^{2}}\right)\right\}+\frac{\tan \theta_{k}}{2} E\left(\Delta^{2} \theta_{k 1}\right)
$$

Note that $\Delta \lambda_{k 1}$ is linear to $N[5]$, thus $E\left(\frac{\Delta \lambda_{k 1}^{2}}{2 \lambda_{k}^{2}}\right)=0$ because of the circularity of the noise. Substitute $E\left(\Delta \lambda_{k 2}\right)$ into (129) to obtain bias final expression as

$$
\begin{aligned}
E\left(\Delta \theta_{k 2}\right)= & \frac{C_{k} \sigma_{n}^{2}}{M} \Im\left\{\mathbf{e}_{k}^{H}\left(\mathbf{A}^{\downarrow^{H}} \mathbf{A}^{\downarrow}\right)^{-1} \mathbf{R}_{s}^{-1} \mathbf{e}_{k}[-(L-K-1)\right. \\
& \left.\left.-\lambda_{k}^{-1} \operatorname{Tr}\left(\mathbf{P}_{\mathbf{A}\rfloor} \mathbf{I}^{\dagger} \mathbf{I}^{\downarrow^{H}}\right)\right]\right\}+\frac{\tan \theta_{k}}{2} E\left(\Delta \theta_{k 1}{ }^{2}\right) .
\end{aligned}
$$




\section{IV.6 NUMERICAL SIMULATIONS}

In this section, we will present numerically the bias performance of DOA estimation algorithms as predicted by our analysis, and compare the results with computer simulations.

The general configuration of the experiments is: a uniform line array of eight sensors (with $d=\lambda_{c} / 2$ ) with two sources at 0.2 and 0.35 radians (angles are mea-

sured with respect to the normal of the array). The signals are $s_{k}(t)=e^{j\left(\omega_{c} t+\phi_{k n}\right)}$, where $\phi_{k n}$ are independent random phase angles uniformly distributed in the interval $(-\pi, \pi)$. Twenty snapshots of array data were simulated for 10 thousand trials. Figure 7 shows bias of DOA estimation versus signal-to-noise-ratio (SNR) for MUSIC.

Figure 8 shows bias of DOA estimation versus signal-to-noise-ratio (SNR) for MinNorm.

Figure 9 shows bias of DOA estimation versus signal-to-noise-ratio (SNR) for ESPRIT.

SNR is defined as

$$
S N R=10 \log \frac{\sigma_{s}^{2}}{\sigma_{n}^{2}}
$$

It is clearly shown from the simulations that our analytical bias for DOA estimation algorithms are accurate and valid over a wide range of SNR.

\section{IV.7 SUMMARY}

This chapter presents bias performance analysis for extrema-searching approach, polynomial-rooting approach and matrix-shifting approach in term of phys- 
ical parameters. The results is verified by the numerical simulations. It has been shown that polynomial-rooting approach is equivalent to extrema-searching approach under the assumption that the DOAs are well separated, which is the first step towards the unification of subspace algorithms. The techniques developed for each approach is quite general which is also applicable to other subspace algorithms. 


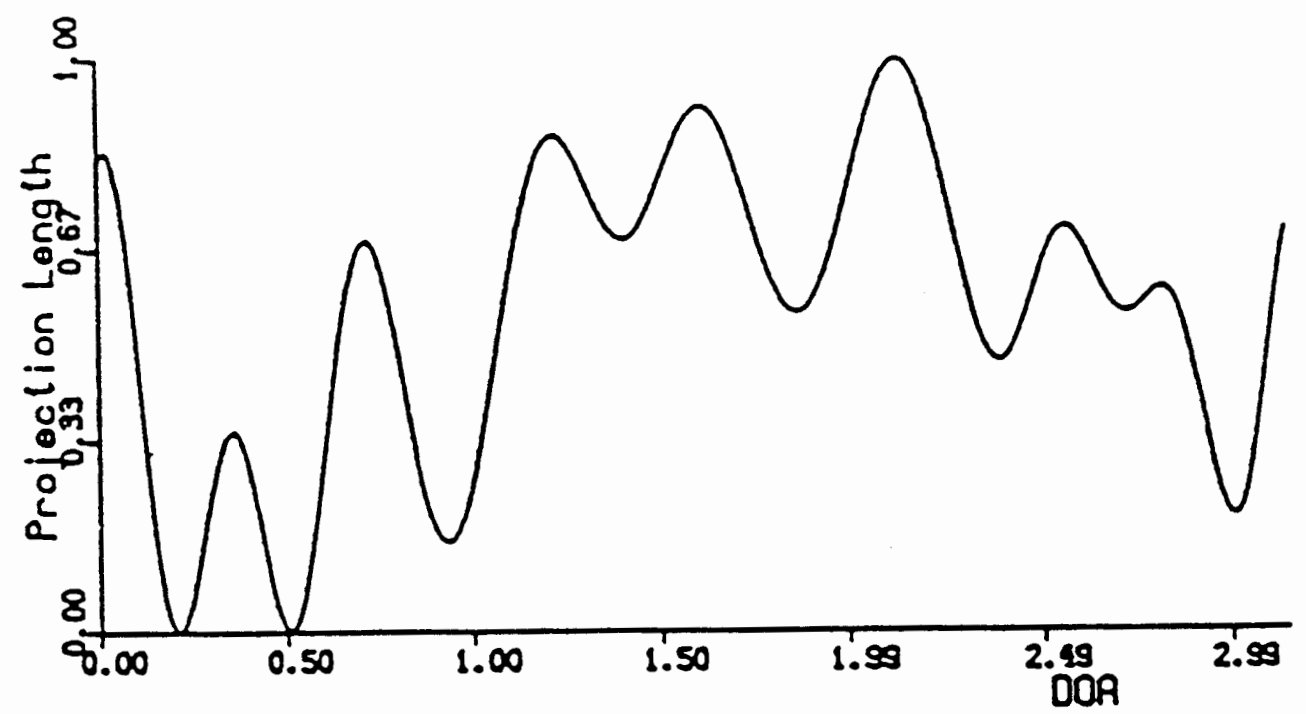

Figure 3. Null-Spectrum Function of Extrema Search Approach. 


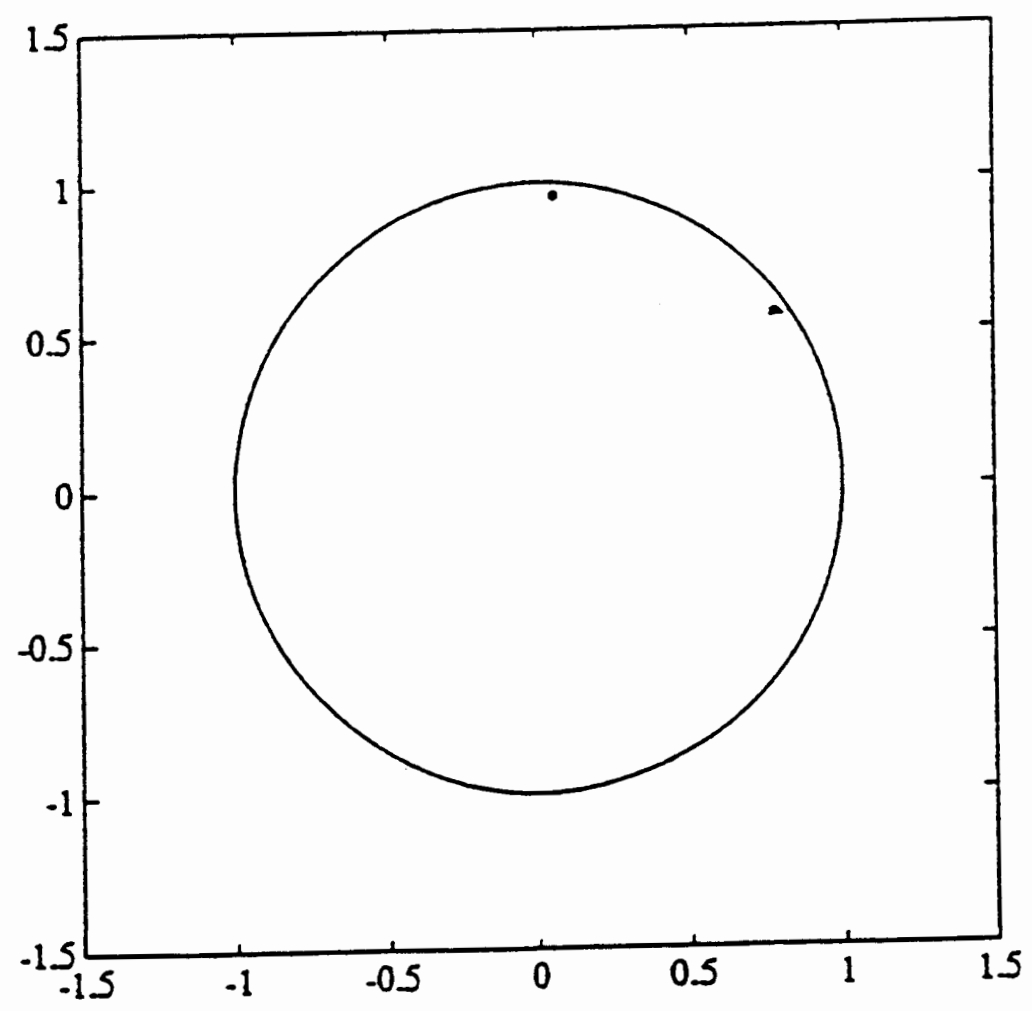

Figure 4. Scattergram of Estimated Roots for Root-MUSIC. 


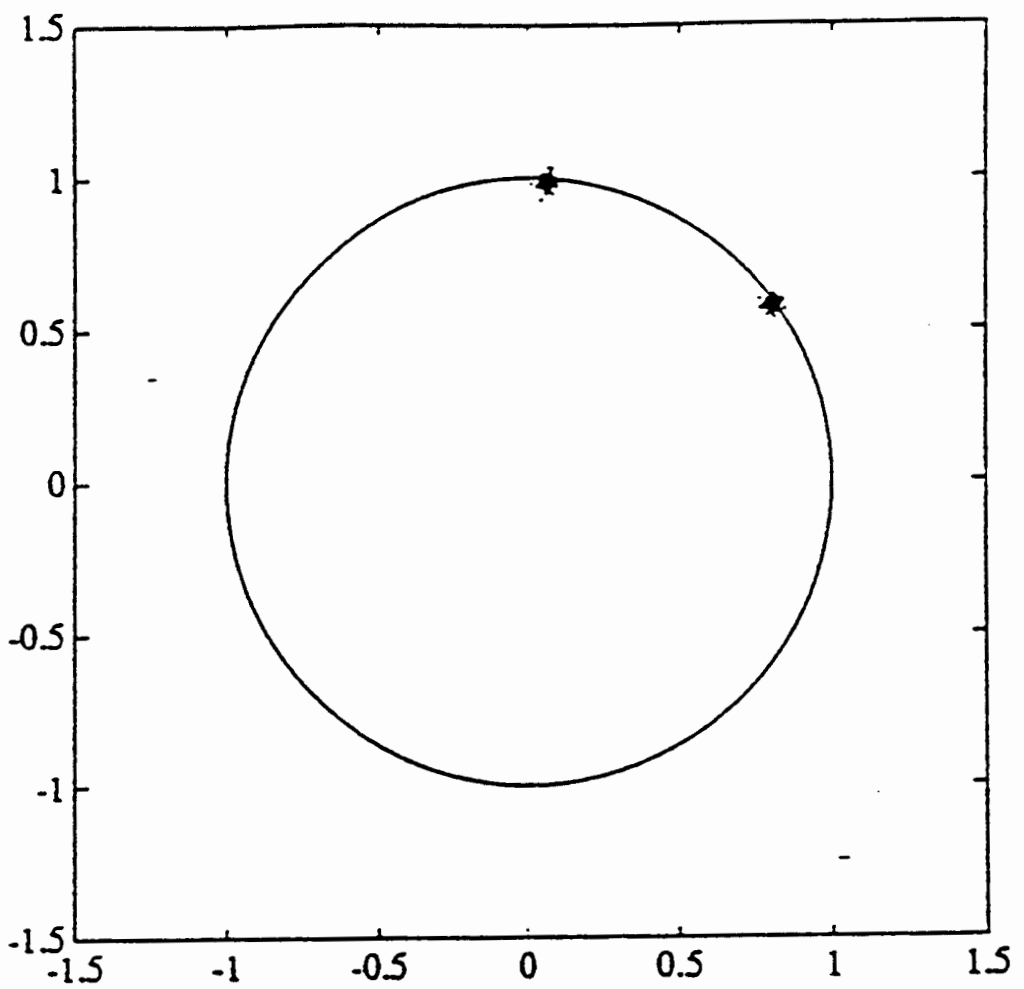

Figure 5. Scattergram of Estimated Roots for Min-Norm. 


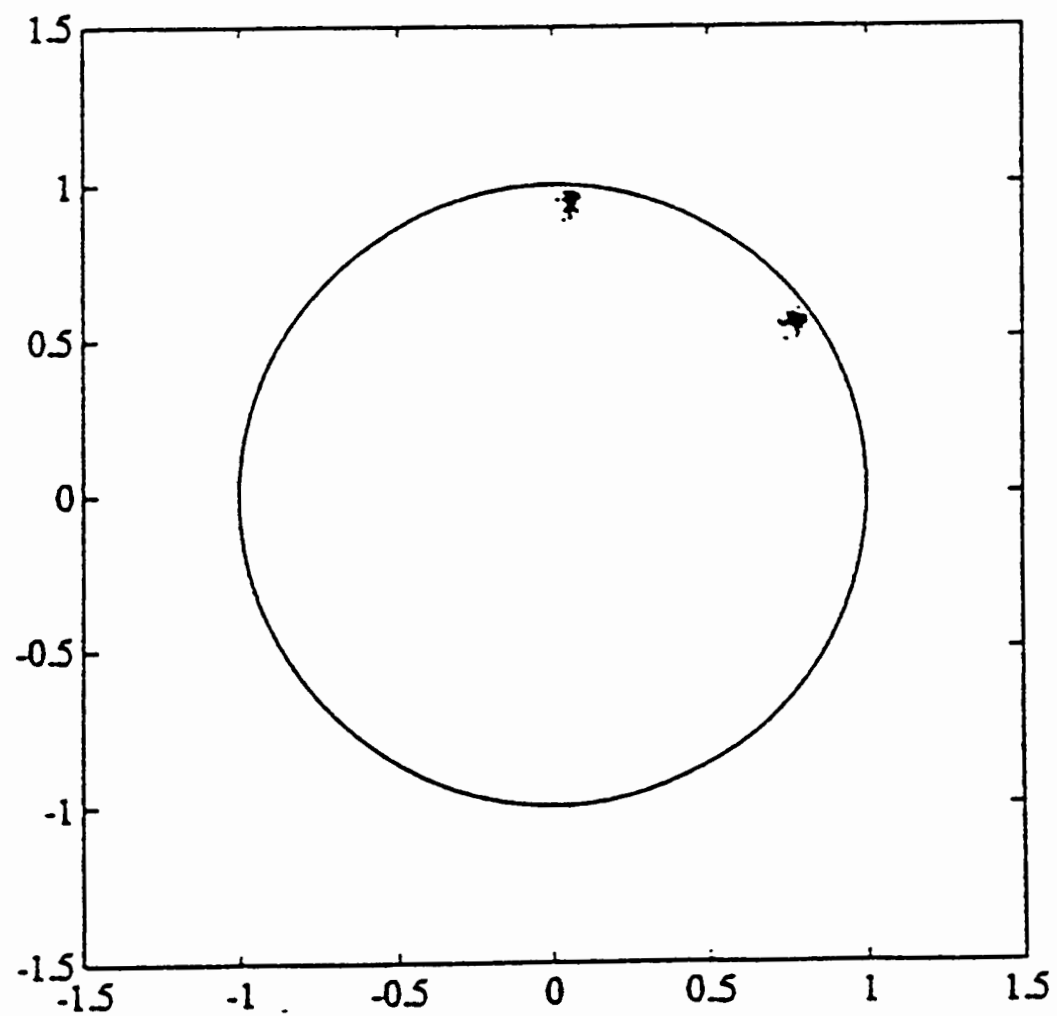

Figure 6. Scattergram of Estimated Eigenvalues for ESPRIT. 


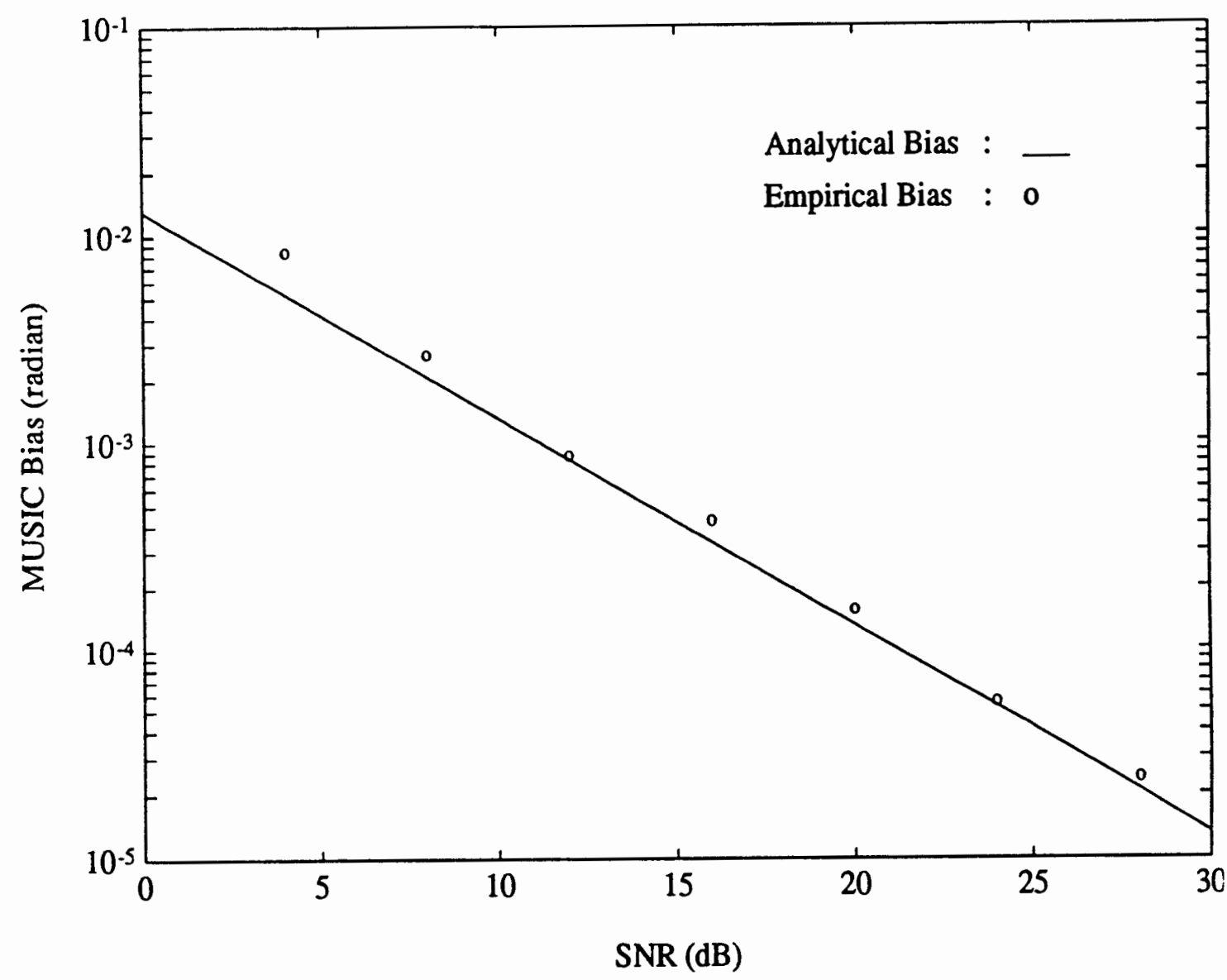

Figure 7. Bias vs. SNR for MUSIC. 


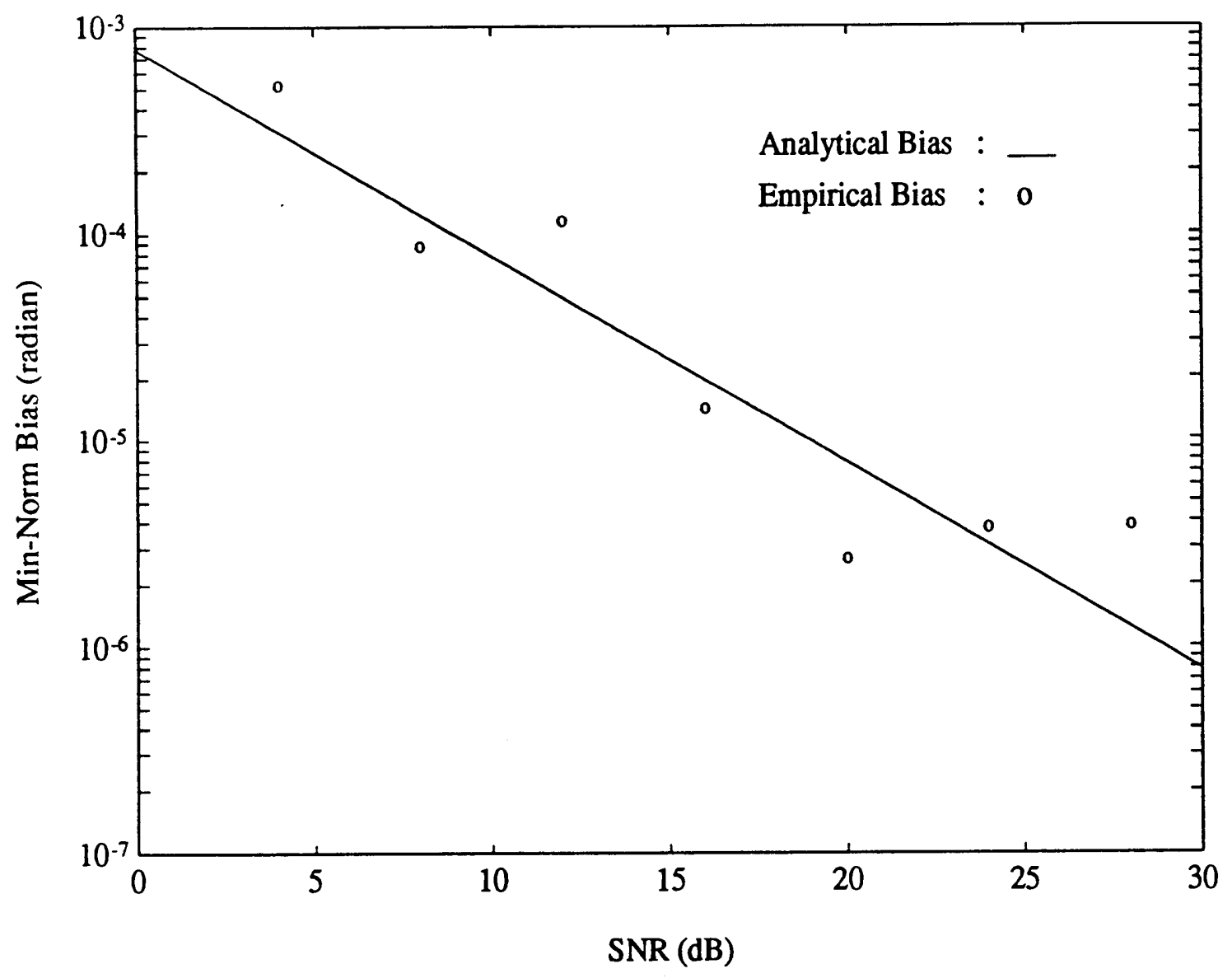

Figure 8. Bias vs. SNR for Min-Norm. 


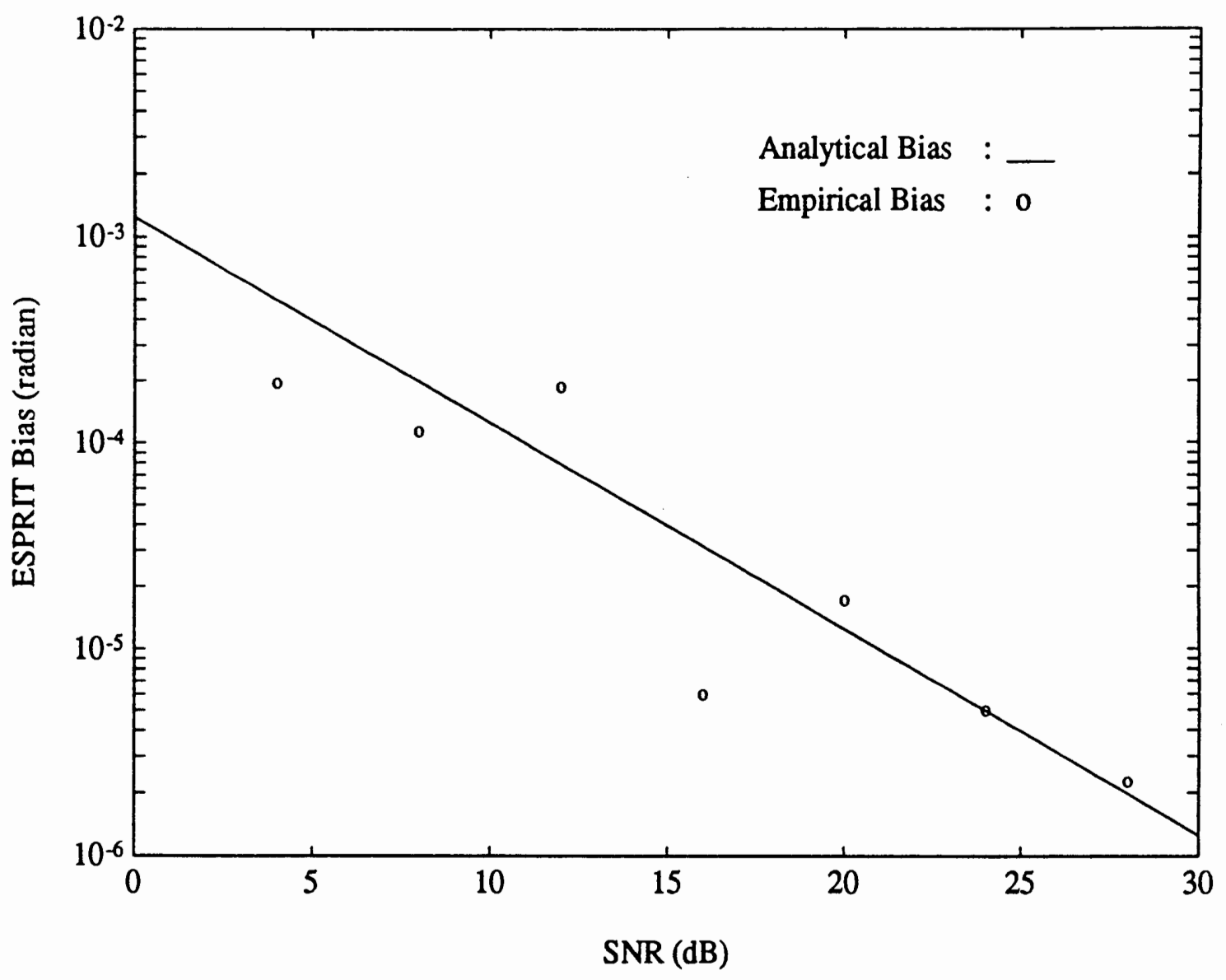

Figure 9. Bias vs. SNR for ESPRIT. 


\section{CHAPTER V \\ UNIFICATION OF BIAS ANALYSES}

\section{V.I INTRODUCTION}

As discussed early on, previous work on individual bias analysis of DOA estimation algorithms makes comparison between different algorithms difficult. In this chapter, we first unify bias expressions of different algorithms in a unified, self-contained fashion then reveal the relationship between the estimated bias and practical factors such as source separation and colherence, numbers of senors and snapshots. Some new insights into different algorithms are drawn through the unified expression.

\section{V.2 UNIFICATION OF BIAS EXPRESSIONS}

The unified, tractable formula for subspace-based DOA algorithms including MUSIC, Min-Norm and ESPRIT is

$$
E\left(\Delta \theta_{k}\right)=\frac{\sigma_{n}^{2}}{M} \circlearrowleft\left\{\mathbf{e}_{k}^{\prime \prime} \mathbf{W}_{B} \mathbf{R}_{s}^{-1} \mathbf{e}_{k}\right\}+D_{k} E\left(\Delta \theta_{k}^{2}\right)
$$

where weighting matrix $\mathrm{W}_{B}$ and constant $D_{k}$ are specified for different algorithms as in Table 2 and $E\left(\Delta 0_{k}^{2}\right)$ is given in [2:3] as

$$
E\left(\Delta O_{k}{ }^{2}\right)=\frac{\mathbf{R}_{s}^{-1}(k, k) \sigma_{n}^{2}}{2 M\left[\mathbf{a}^{(1)}\left(O_{k}\right)^{H I} \Omega_{o} \mathbf{W}_{M} \Omega_{o} \mathbf{a}^{(1)}\left(0_{k}\right)\right]} .
$$

Here, $\boldsymbol{\Omega}_{o}$ is the projection matrix which satisfies $\Omega_{o}=\mathbf{U}_{o} \mathbf{U}_{o}^{H}=\mathbf{I}-\mathbf{A}\left(\mathbf{A}^{H} \mathbf{A}\right)^{-1} \mathbf{A}^{H}$ and the weighting matrix $\mathbf{W}_{M}$ is specialized for different algorithms as in table 3 . In [23], $\alpha^{H}$ is given as $\Omega^{H}=C_{k} \cdot \mathbf{e}_{k}^{\prime \prime}\left(\mathbf{A}^{1^{\dagger}} \mathbf{I}^{1}-\mathbf{A}^{1^{\dagger}} \mathbf{I}^{1}\right)$. 


\section{V.3 THEORETICAL STUDY}

In this section, we will study and compare the performance of MUSIC, MinNorm and ESPRIT based on the analytical bias (132), MSE [23] and variance as functions of SNR, number of senors and snapshots. Since we are interested in the performance of algorithms at low SNR, insufficient snapshots and small number of available senors, which are the limitations in array applications, we will set two of three parameters to small numbers while changing the third one at a large range. The relationship between bias, mean square error (MSE) and variance (VAR) is shown as

$$
\begin{aligned}
V A R & =E\left\{[\tilde{\theta}-E(\tilde{\theta})]^{2}\right\} \\
& =E\left\{[(\tilde{\theta}-\theta)-E(\Delta \theta)]^{2}\right\} \\
& =E\left[(\tilde{\theta}-\theta)^{2}\right]-E^{2}(\Delta \theta) \\
& =M S E-(\text { bias })^{2} .
\end{aligned}
$$

\section{V.3.1 Bias, MSE and Variance vs. number of senors}

The general configuration of the experiments is the same as described in Chapter 4. SNR is set to be 10 and snapshots is chosen to be 20 .

Figure 10 shows bias for MUSIC, Min-Norm and ESPRIT vs. number of senors.

Figure 11 shows MSE for MUSIC, Min-Norm and ESPRIT vs. number of senors. Figure 12 shows variance for MUSIC, Min-Norm and ESPRIT vs. number of senors.

Observations:

- MUSIC has the largest bias which is comparable to MSE when the number of 
senors is small and bias of MUSIC decreases fastest with increase of number of senors.

- The bias is not monotonically decreasing for MUSIC and Min-Norm with increase of number of senors.

- MUSIC has the smallest MSE which was proved in [23].

- MSE for Min-Norm and ESPRIT is not monotonically decreasing with increase of number of senors.

- Variance for all three algorithms become closer to MSE when number of senors increases, which can be explained by the dominant MSE in variance while the bias is negligible.

- MUSIC performs best when the number of senors is sufficiently large since both variance and MSE are the smallest and bias is negligible.

\section{V.3.2 Bias, MSE and VAR vs. SNR}

The general configuration of the experiments is the same as described in Chapter 4. Here, senor number is chosen to be 8 and we assume 20 snapshots available.

Figure 13 shows bias for MUSIC, Min-Norm and ESPRIT vs. SNR.

Figure 14 shows MSE for MUSIC, Min-Norm and ESPRIT vs. SNR.

Figure 15 shows variance for MUSIC, Min-Norm and ESPRIT vs. SNR. Observations:

- At low SNR, MUSIC has large bias which is comparable to MSE while MinNorm and ESPRIT have negligible bias compared to MSE. 
- MUSIC always has the smallest MSE.

- Bias as a function of $\sigma_{n}^{2}$ decreases faster than MSE (function of $\sigma_{n}^{2}$ ) resulting in closer variance to MSE when SNR increases.

- MUSIC has the best performance at high SNR owing to lowest variance and MSE as well as the negligible bias.

\section{V.3.3 Bias, VAR and MSE vs. snapshots}

The general configuration of the experiments is the same as described in Chapter 4. SNR is set to 10 and senor number is equal to 8 . Since the snapshots plays a similar role to that of SNR in Bias, Variance and MSE expressions, we expect to observe performance close to what we see from above.

Figure 16 shows bias for MUSIC, Min-Norm and ESPRIT vs. snapshots.

Figure 17 shows variance for MUSIC, Min-Norm and ESPRIT vs. snapshots.

Figure 18 shows MSE for MUSIC, Min-Norm and ESPRIT vs. snapshots.

Observation:

- Compared to MSE, bias of MUSIC is very large at small number of snapshots while bias of Min-Norm and ESPRIT are alway small enough to be neglected.

- MSE for MUSIC is the smallest.

- At large number of snapshots, variance is close to MSE due to the negligible bias.

\section{V.4 CONCLUSION}

In summary, we have presented unified bias expression for subspace-based DOA estimation algorithms including MUSIC, Min-Norm and Subspace Rotation. 
The expression is obtained through the exploration of the relationship between the estimated DOAs and the underlying estimated subspace. The major work includes:

- deriving the second-order subspace perturbations induced by noise.

- equating DOA estimation bias for extrema searching and polynomial rooting algorithms .

- unifying DOA estimation bias of subspace-based algorithms in term of signal, noise and array characters (physical parameters).

- providing additional insights into the performance of DOA algorithms from the unified bias formula. 
TABLE II

WEIGHTING MATRIX FOR BIAS EXPRESSION

\begin{tabular}{|c|c|}
\hline & $\mathbf{W}_{B}$ \\
\hline \hline MUSIC & $-\frac{j(L-K-1)}{\left\|\mathbf{a}^{(1)}\left(\theta_{k}\right)^{H} \mathbf{\Omega}_{o}\right\|^{2}} \mathbf{A}^{(1) H}\left(\mathbf{A}^{\dagger}\right)^{H}$ \\
\hline Min-Norm & $-\frac{j(L-K-1)}{\left\|\mathbf{a}^{(1)}\left(\theta_{k}\right)^{H} \mathbf{\Omega}_{o} \mathbf{e}_{1}\right\|^{2}} \mathbf{A}^{(\mathbf{1}) H} \mathbf{\Omega}_{o} \mathbf{e}_{1} \mathbf{e}_{1}^{H}\left(\mathbf{A}^{\dagger}\right)^{H}$ \\
\hline ESPRIT & $-C_{k}\left\{(L-K-1)+\lambda_{k}^{-1} \operatorname{Tr}\left(\mathbf{P}_{A l} \mathbf{I}^{\dagger} \mathbf{I}^{\downarrow^{H}}\right)\right\}\left(\mathbf{A}^{\downarrow^{H}} \mathbf{A}^{\downarrow}\right)^{-1}$ \\
\hline
\end{tabular}

TABLE III

CONSTANT SPECIFICATION FOR BIAS EXPRESSION

\begin{tabular}{|c|c|}
\hline & $D_{k}$ \\
\hline \hline MUSIC & $-\frac{\Re\left(\mathbf{a}^{(1)}\left(\theta_{k}\right)^{H} \mathbf{\Omega}_{o \mathbf{a}} \mathbf{a}^{(2)}\left(\theta_{k}\right)\right\}}{2\left\|\mathbf{a}^{(1)}\left(\theta_{k}\right)^{H} \Omega_{o}\right\|^{2}}$ \\
\hline Min-Norm & $-\frac{\Re\left\{\mathbf{a}^{(1)}\left(\theta_{k}\right)^{H} \Omega_{o} \mathbf{e}_{1} \mathbf{e}_{1}^{H} \Omega_{o} \mathbf{a}^{(2)}\left(\theta_{k}\right)\right\}}{2\left\|\mathbf{a}^{(1)}\left(\theta_{k}\right)^{H} \Omega_{o} \mathbf{e}_{1}\right\|^{2}}$ \\
\hline ESPRIT & $\frac{\tan \theta_{k}}{2}$ \\
\hline
\end{tabular}

TABLE IV

WEIGHTING MATRIX OF MSE EXPRESSION

\begin{tabular}{|c|c|c|c|}
\hline & MUSIC & Min-Norm & SSR \& ESPRIT \\
\hline \hline $\mathbf{W}_{M}$ & $\mathbf{I}$ & $\frac{\boldsymbol{\Omega}_{o} \mathbf{e}_{1} \mathbf{e}_{1}^{H} \boldsymbol{\Omega}_{o}}{\left\|\mathbf{e}_{1}^{H} \boldsymbol{\Omega}_{o}\right\|^{2}}$ & $\frac{\alpha \alpha^{H}}{\|\alpha\|^{2}}$ \\
\hline
\end{tabular}




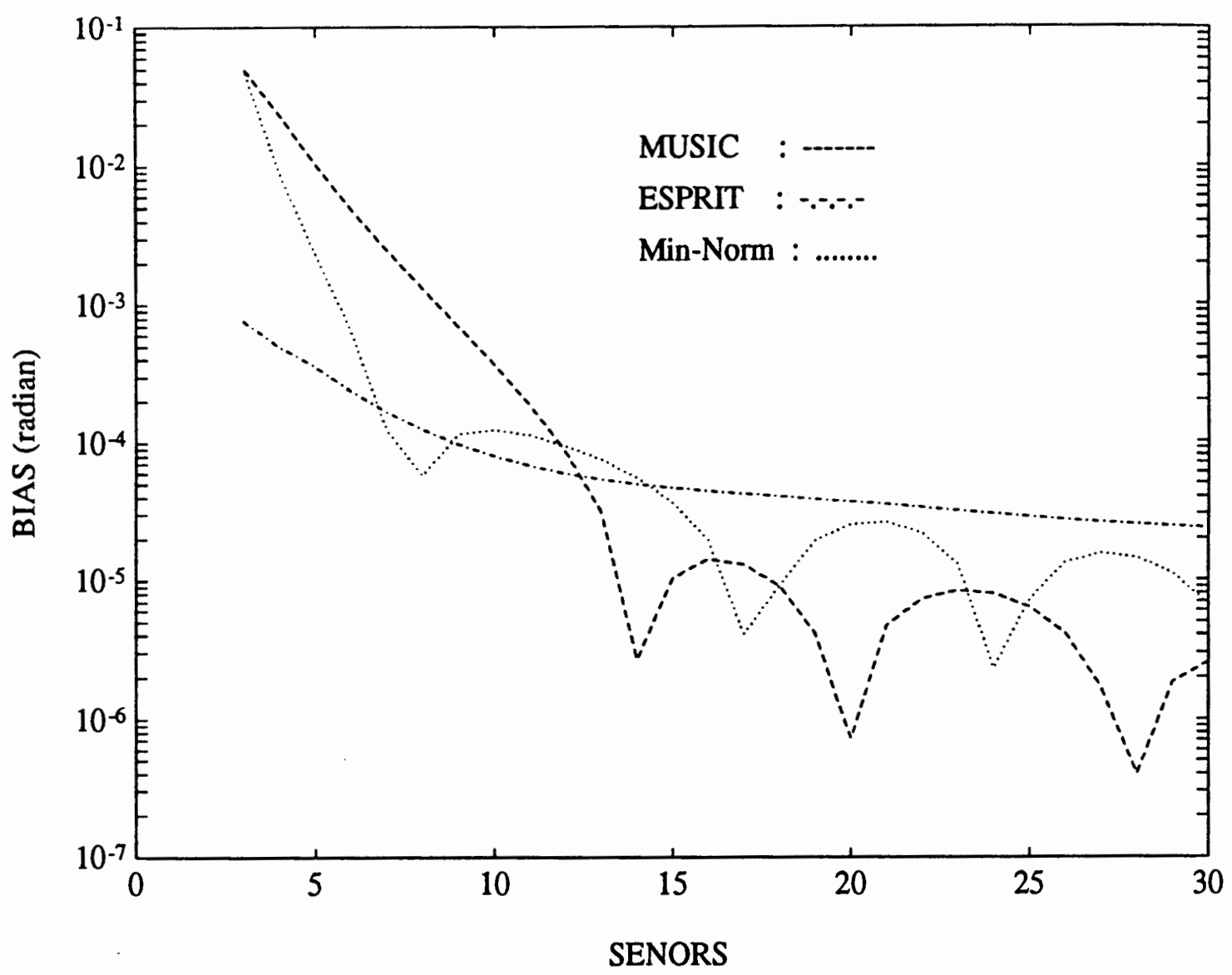

Figure 10. Bias vs. Senors. 


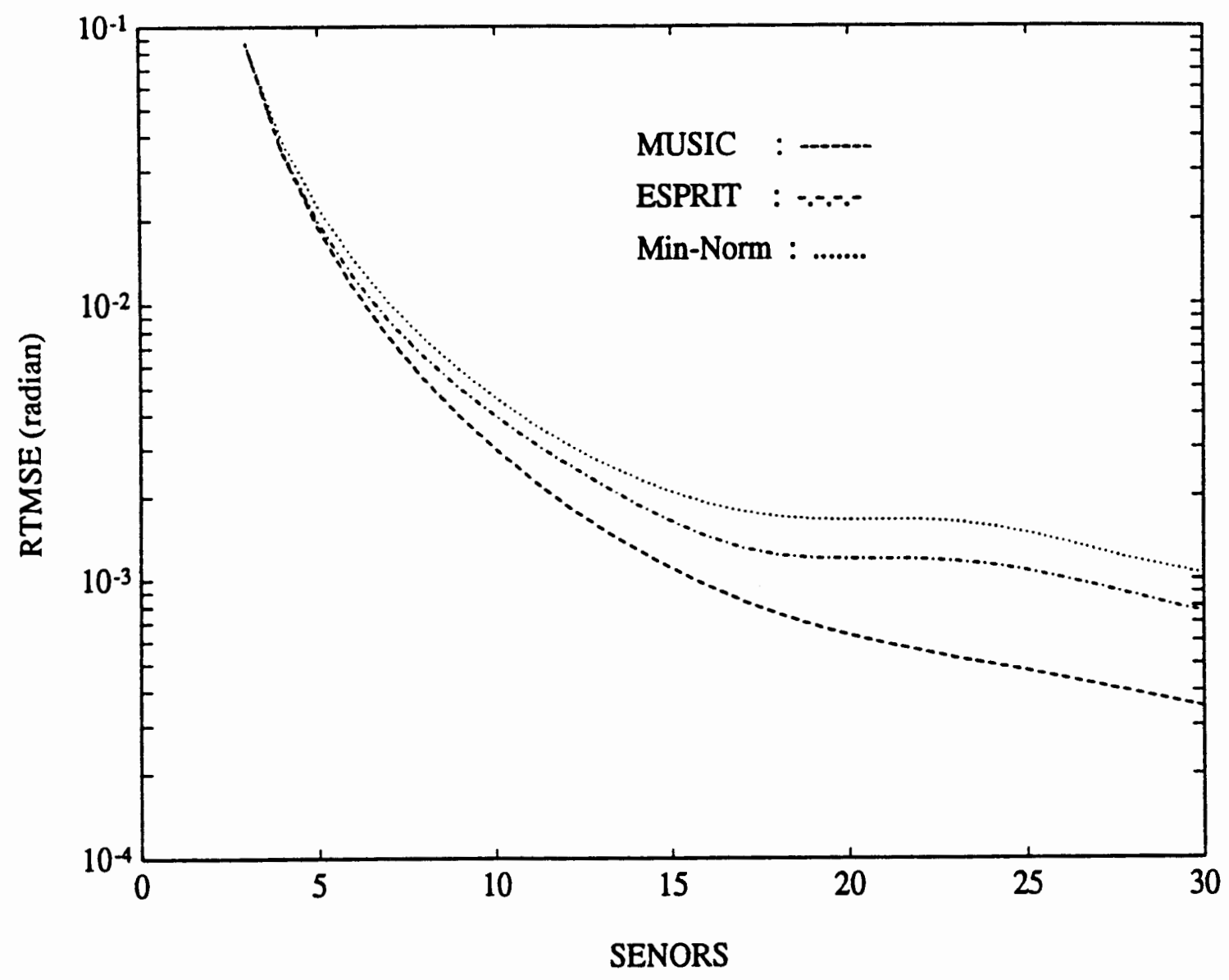

Figure 11. MSE vs. Senors. 


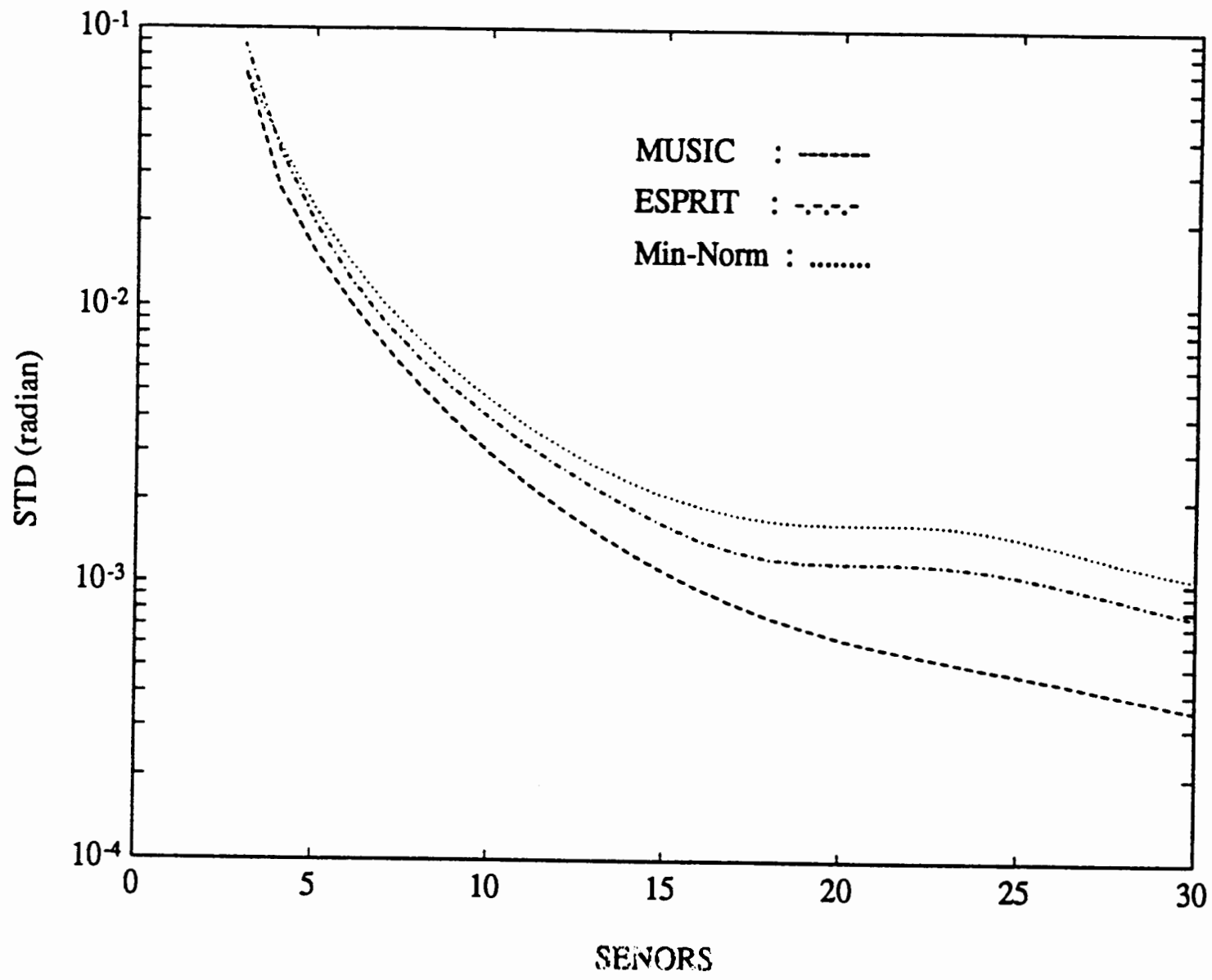

Figure 12. Variance vs. Senors. 


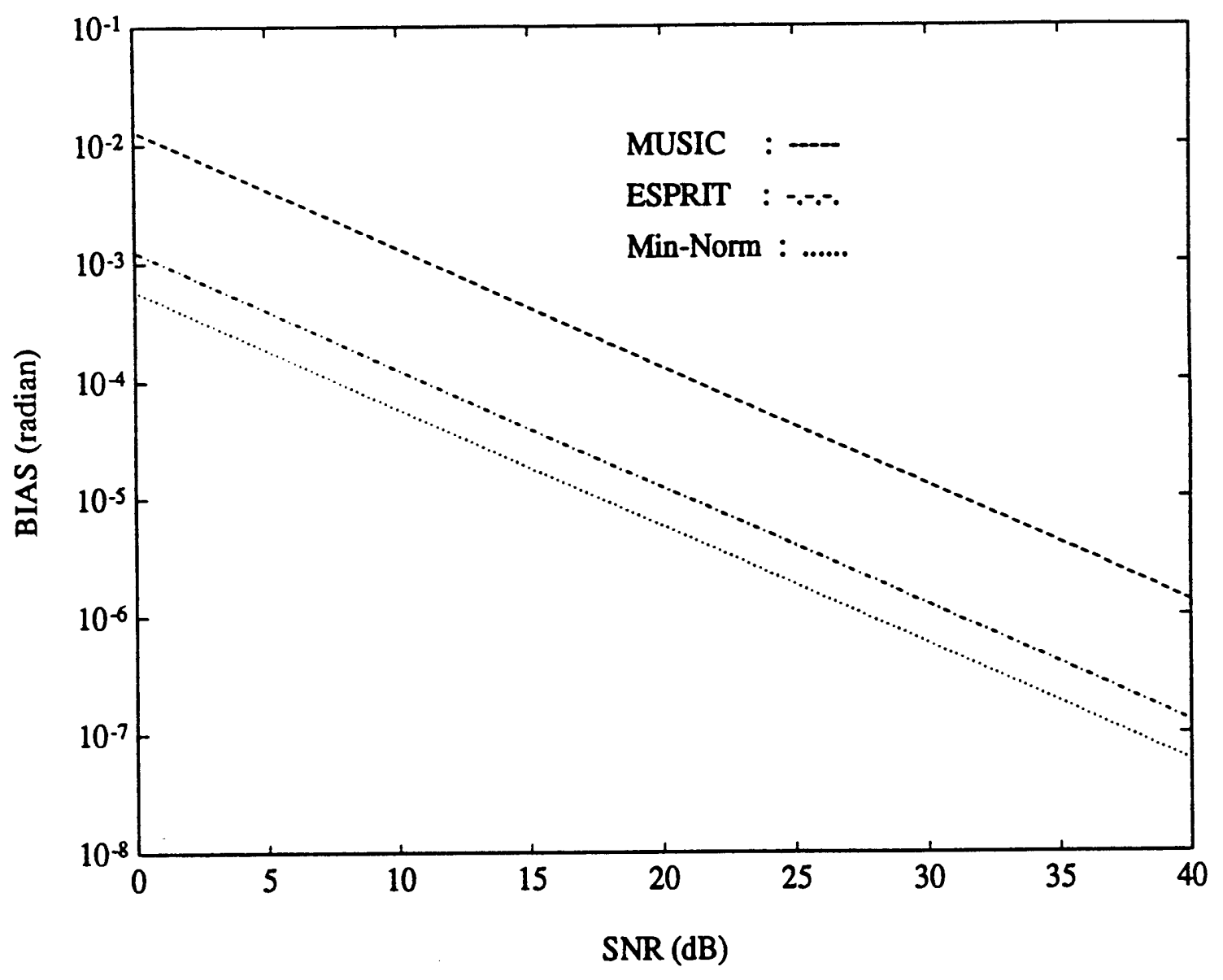

Figure 13. Bias vs. SNR. 


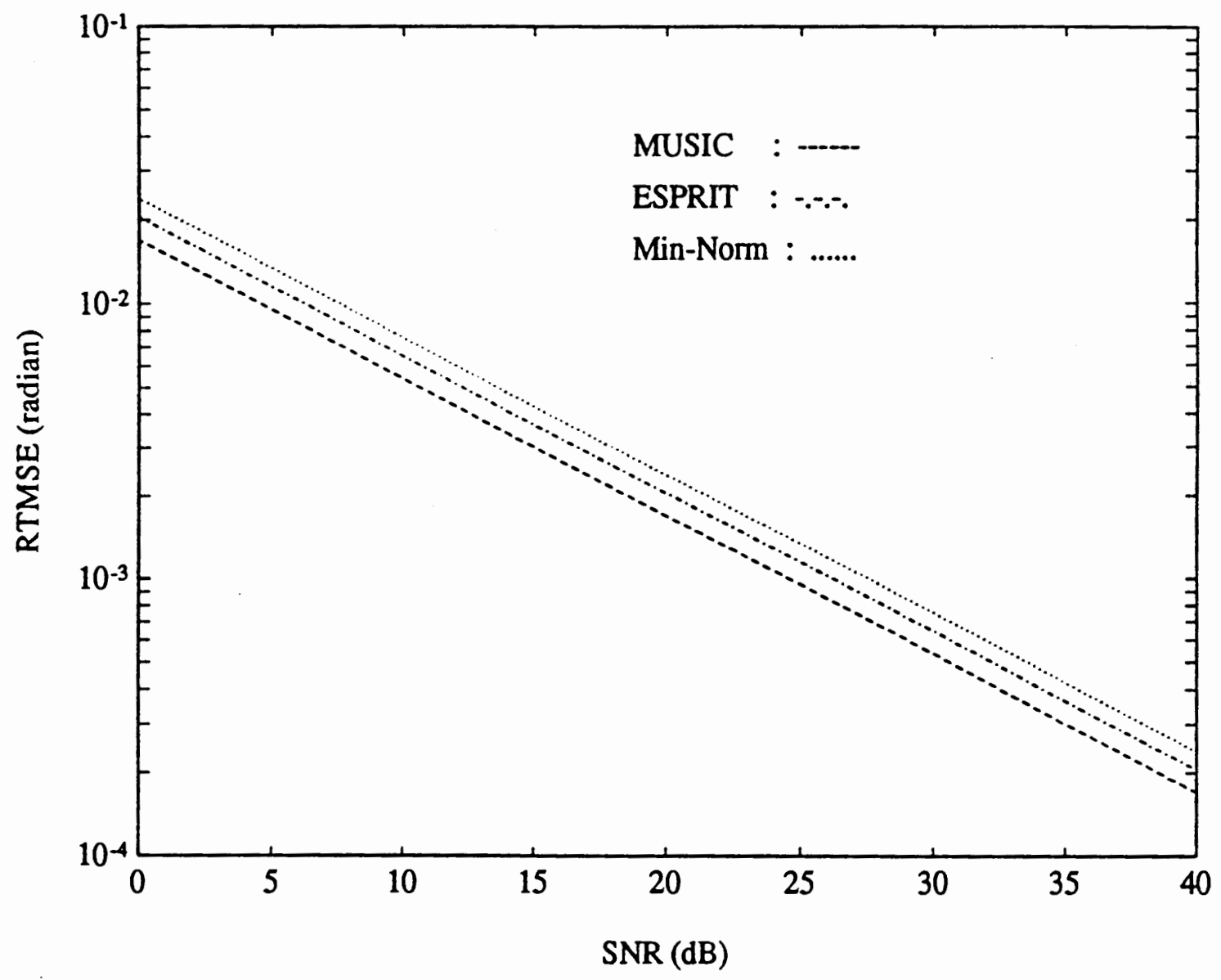

Figure 14. MSE vs. SNR. 


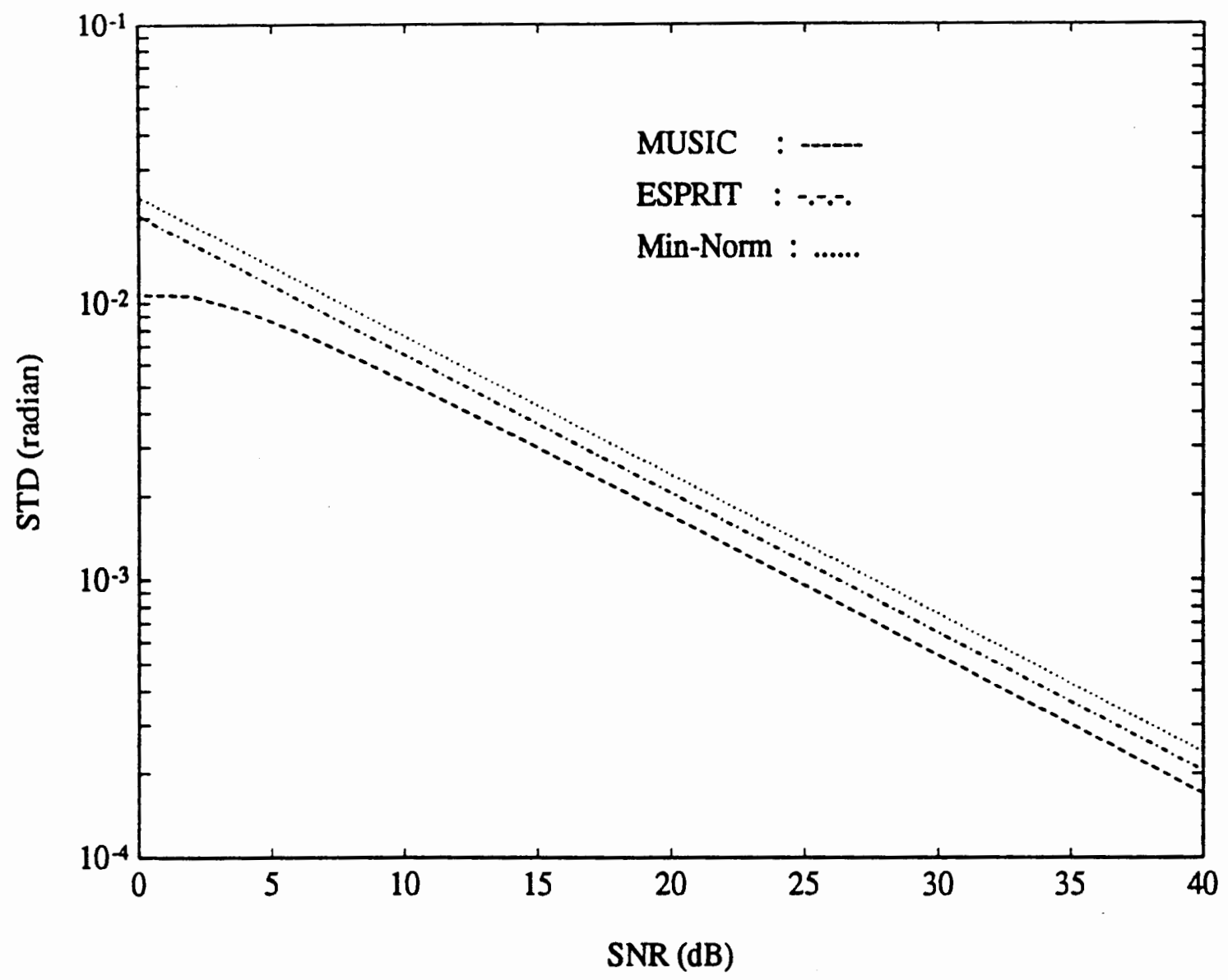

Figure 15. Variance vs. SNR. 


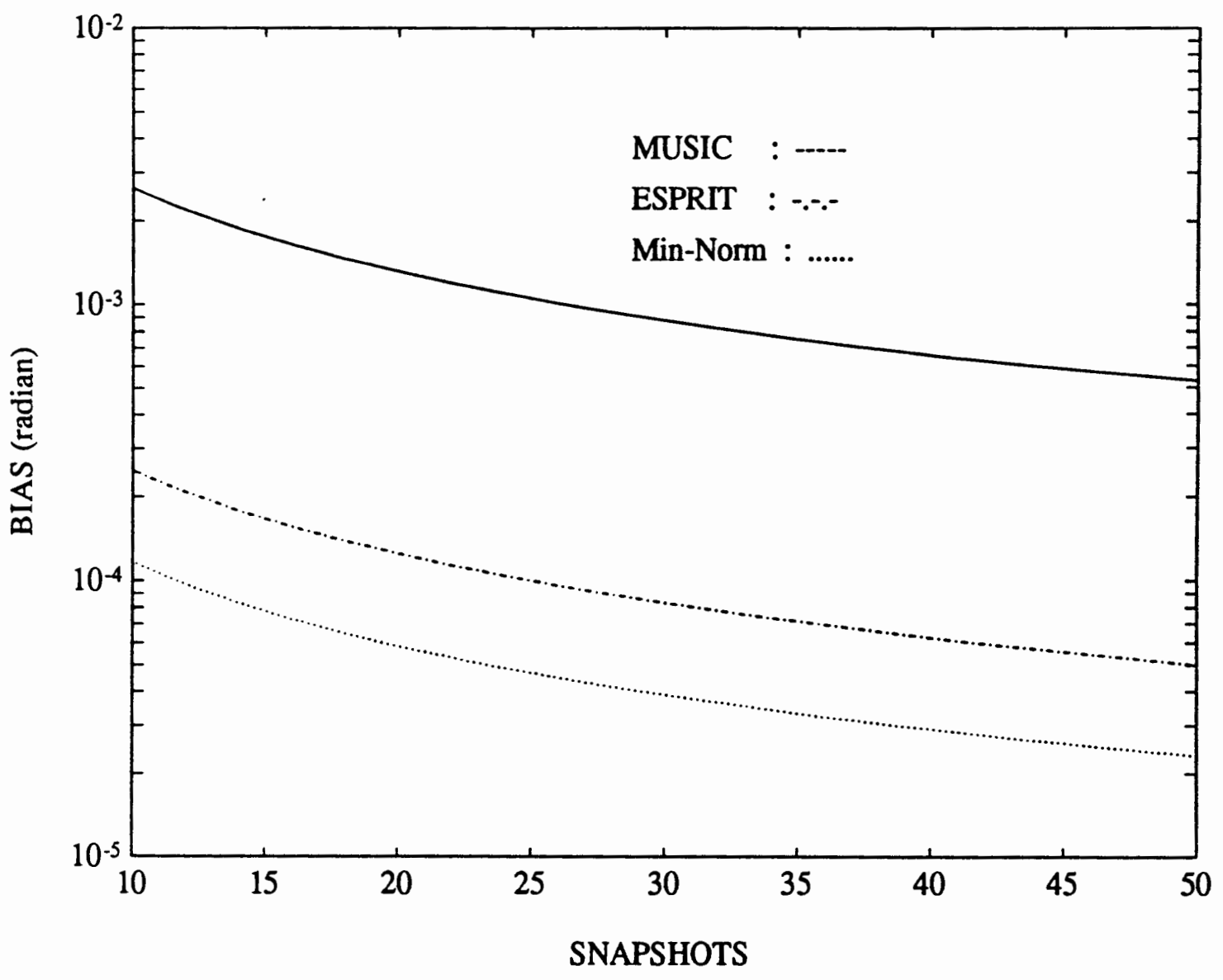

Figure 16. Bias vs. Snapshots. 


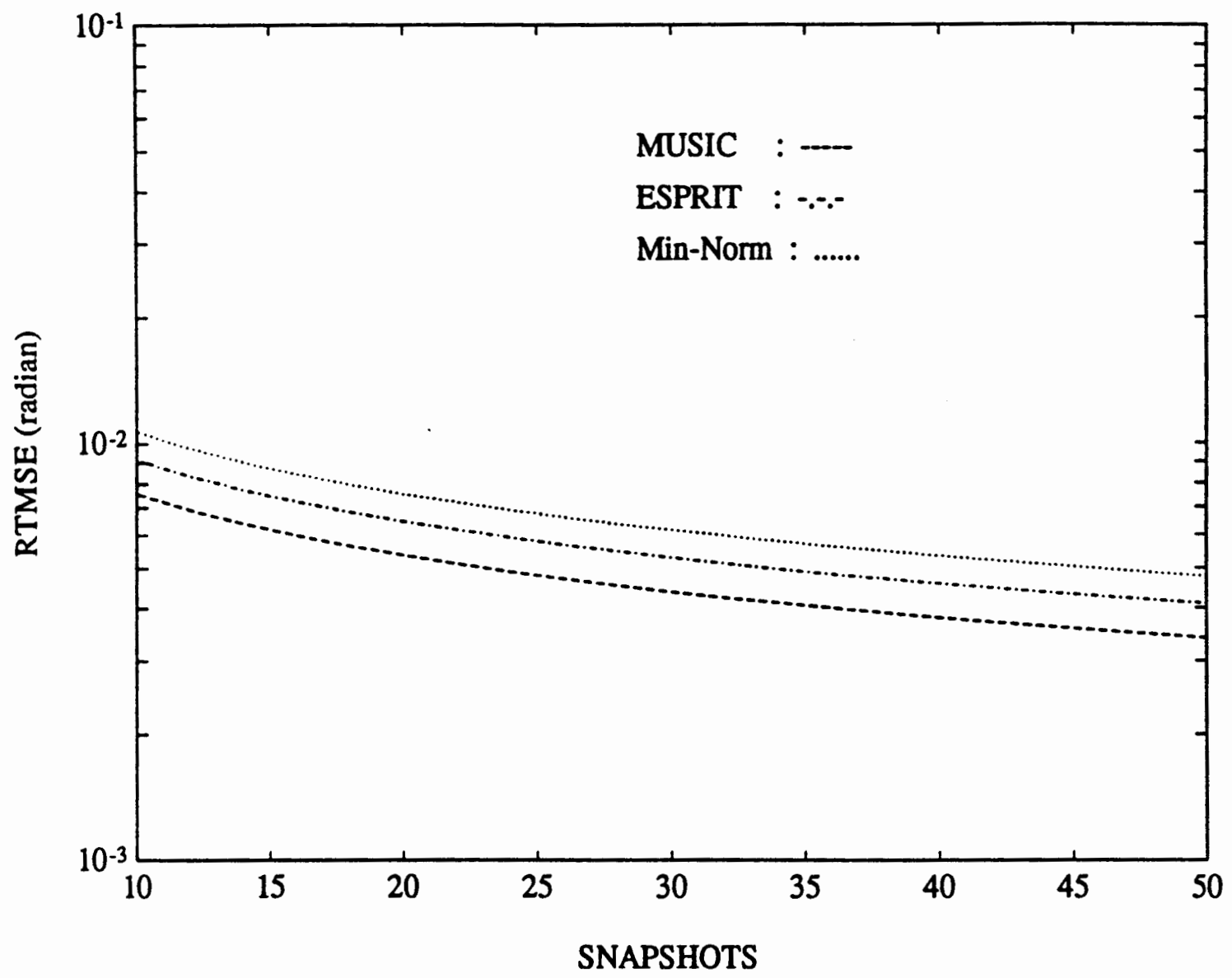

Figure 17. MSE vs. Snapshots. 


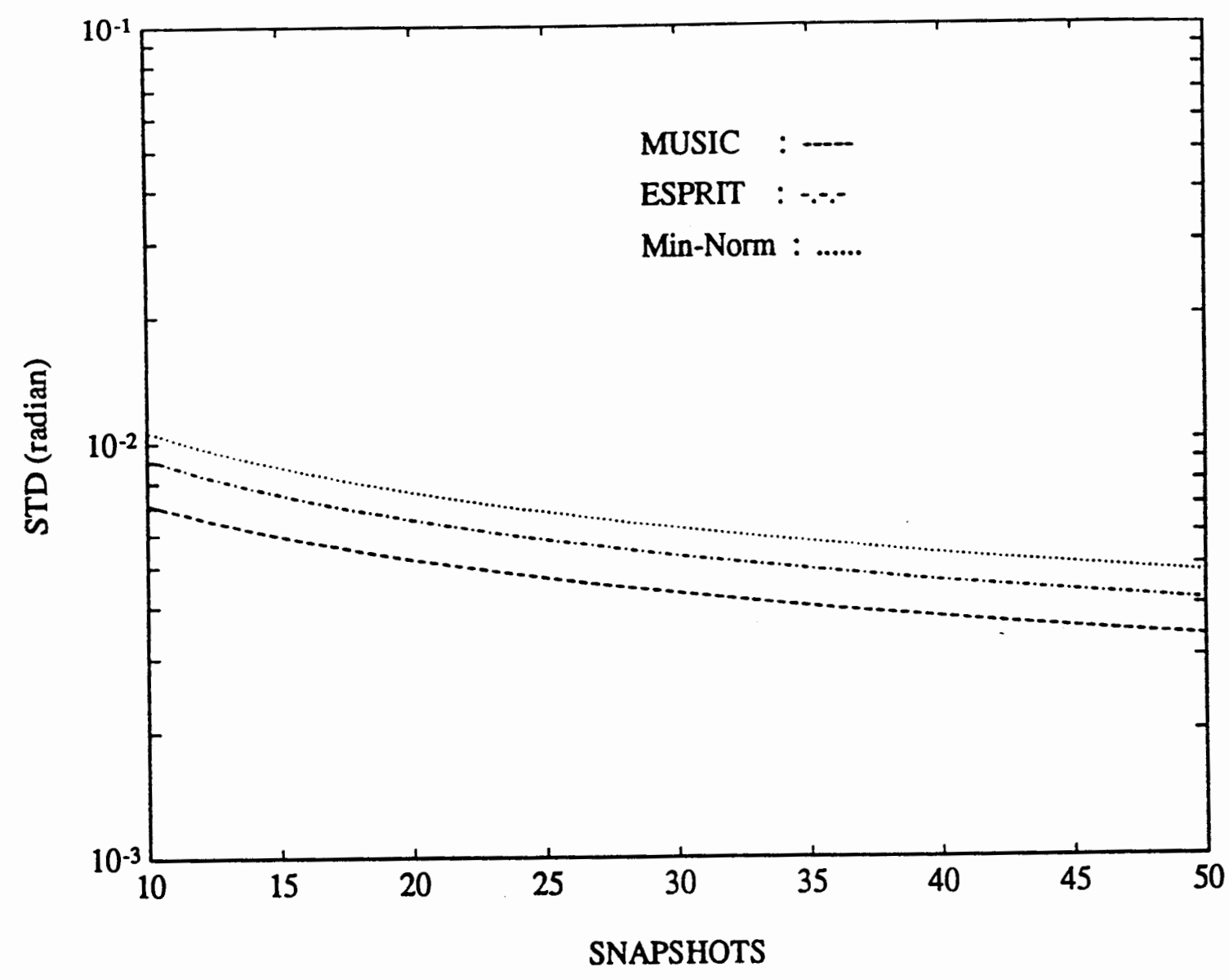

Figure 18. Variance vs. Snapshots. 


\section{REFERENCES}

[1] F. $\mathrm{Li}$ and Y. Lu, "Bias analysis for DOA estimation algorithms," in Proc. 6th IEEESP Workshop on Statistical Signal $\mathcal{B}$ Array Processing, (Victoria, BC. Canada),pp. 314-317, October 1992.

[2] Y. Lu and F. Li, "Unified bias for DOA estimation algorithms," in Proc. ICASSP'g9, (Minneapolis, MN), pp. 376-379, April 1993.

[3] R. Roy, A. Paulaj, and T. Kailath, "Estimation of signal parameters via rotational invariance techniques - ESPRIT," in Proc. IEEE MILCON, pp. 41.6.1-41.6.5, 1986.

[4] M. Kaveh and A. J. Barabell, "The statistical performance of the MUSIC and the Minimum-Norm algorithms in resolving plane-waves in noise," IEEE Transactions on Acoustics, Speech, and Signal Processing, vol. ASSP-34, pp. 331-340, April 1986.

[5] F. Li and R. J. Vaccaro, "Unified analysis for DOA estimation algorithms in array signal processing," Signal Processing, vol. 22, pp. 147-169, November 1991.

[6] H. Wang and G. H. Wakefield, "Non-asymptotic performance analysis of eigenstructure spectral methods," in Proc. IEEE ICASSP'89, (Glasgow, UK), pp. 2591-2594, May 1989.

[7] X.-L. Xu and K. M. Buckley, "Bias and variance analysis of MUSIC location estimates," in Proc. 5th IEEE ASSP Workshop on Spectrum Estimation $\mathcal{B}$ Modeling, (Rochester, NY), pp. 332-336, October 1990.

[8] F. Li, A Unified Performance Analysis of Subspace-Based DOA Estimation Algorithms. PhD thesis, University of Rhode Island, Kingston, RI, 1990.

[9] R. O. Schmidt, "Multiple emitter location and signal parameter estimation," in Proc. RADC Spectral Estimation Workshop, (Griffiss AFB, NY), pp. 243-258, 1979.

[10] R. Kumaresan and D. W. Tufts, "Estimating the angles of arrival of multiple plane waves," IEEE Transactions on Aerospace and Electronic Systems, vol. AES19, pp. 134-139, January 1983.

[11] F. Li, R. J. Vaccaro, and D. W. Tufts, "Min-Norm Linear Prediction for arbitrary sensor array," in Proc. IEEE ICASSP'89, (Glasgow, UK), pp. 2613-2616, May 1989.

[12] A. J. Barabell, "Improving the resolution performance of eigenstructure-based direction-finding algorithm," in Proc. IEEE ICASSP'83, pp. 336-339, 1983. 
[13] A. Paulraj, R. Roy, and T. Kailath, "Estimation of signal parameters via rotational invariance techniques - ESPRIT," in Proc. $19^{\text {th }}$ Asilomar Conf. on Signals, Systems and Computers, (Pacific Grove, CA), pp. 83-89, November 1985.

[14] S. Y. Kung, D. V. Bhaskar Rao, and K. S. Arun, "New state space and singular value decomposition based approximate modeling methods for harmonic retrieval," in IEEE 2nd ASSP Workshop on Spectral Estimation E Modeling, pp. 266-271, August 1983.

[15] S. Y. Kung, K. S. Arun, and D. V. Bhaskar Rao, "State-space and singular-value decomposition-based approximation methods for the harmonic retrieval problem," $J$. Opt. Soc. Am., vol. 73, pp. 1799-1811, December 1983.

[16] R. Foka and S. Y. Kung, "A novel optimum energy solution in iterative constrained restoration,” in Proc. EUSIPCO'88, pp. 1041-1044, Science Publishers B. V., 1986.

[17] R. Foka, "Comparison of high resolution spectral methods based on SVD," in Proc. IEEE ICASSP'87, (Dallas, TX), pp. 2233-2236, April 1987.

[18] H. Ouibrahim, D. D. Weiner, and T. K. Sarkar, "A general approach to directionfinding," in Proc. IEEE MILCON, pp. 41.4.1-41.4.5, 1986.

[19] H. Ouibrahim, D. D. Weiner, and T. K. Sarkar, "Matrix pencil approach to angle of arrival estimation," in Proc. $20^{\text {th }}$ Asilomar Conf. on Signals, Systems and Computers, (Pacific Grove, CA), pp. 203-206, November 1986.

[20] H. Ouibrahim, D. D. Weiner, and T. K. Sarkar, "A general approach to directionfinding," IEEE Transactions on Acoustics, Speech, and Signal Processing, vol. ASSP. 36, pp. 610-612, April 1988.

[21] Y. Hua and T. K. Sarkar, "Matrix pencil method and its performance," in Proc. IEEE ICASSP'88, (New York, NY), pp. 2476-2479, April 1988.

[22] F. Li and R. J. Vaccaro, "Analysis of MUSIC and Min-Norm for arbitrary array geometry," IEEE Transactions on Aerospace and Electronic Systems, vol. AES-26, pp. 976-985, November 1990.

[23] F. Li, H. Liu, and R. J. Vaccaro, "Performance analysis for DOA estimation algorithms using physical parameters," in Proc. ICASSP'92, vol. II, (San Francisco, CA), pp. 537-540, March 1992.

[24] P. Lancaster and M. Tismentsky, The Theory of Matrices. New York, NY: Academic Press, second ed., 1978. 
APPENDIX A

(CALCULATION of (6T) 
Assuming $\mathrm{N}$ is a noise matrix $(L \times M)$ as defined early which can be written as

$$
\mathbf{N}=\left[\mathbf{n}_{1} \cdots \mathbf{n}_{i} \cdots \mathbf{n}_{M}\right]
$$

where the column vector

$$
\mathbf{n}_{i}=\left[\begin{array}{c}
n_{i 1} \\
\vdots \\
n_{i L}
\end{array}\right]
$$

$\mathrm{U}(M \times N)$ and $\mathrm{V}(M \times N)$ are any matrices which can be written as

$$
\mathrm{U}=\left[\begin{array}{c}
\mathbf{u}_{1} \\
\vdots \\
\mathbf{u}_{i} \\
\vdots \\
\mathbf{u}_{M}
\end{array}\right]
$$

where the row vector

$$
\mathbf{u}_{i}=\left[u_{i 1} \cdots u_{i N}\right]
$$

and

$$
\mathbf{V}=\left[\begin{array}{c}
\mathbf{v}_{1} \\
\vdots \\
\mathbf{v}_{j} \\
\vdots \\
\mathbf{v}_{M}
\end{array}\right]
$$

where the row vector

$$
\mathbf{v}_{j}=\left[v_{j 1} \cdots v_{j N}\right]
$$

where the row vector

$$
\mathbf{u}_{j}=\left[u_{j 1} \cdots u_{j N}\right]
$$

thus

$$
\begin{aligned}
E\left(\mathrm{NUV}^{H} \mathbf{N}^{H}\right) & =E\left(\sum_{i=1}^{M} \mathbf{n}_{i} \mathbf{u}_{i} \sum_{j=1}^{M} \mathbf{v}_{j}^{H} \mathbf{n}_{j}^{H}\right) \\
& =\sum_{i=1}^{M} \sum_{j=1}^{M} \mathbf{u}_{i} \mathbf{v}_{j}^{H} E\left(\mathbf{n}_{i} \mathbf{n}_{j}^{H}\right)
\end{aligned}
$$




$$
\begin{aligned}
& =\sum_{i=1}^{M} \sum_{j=1}^{M} \mathbf{u}_{i} \mathbf{v}_{j}^{H} \sigma_{n}^{2} \delta(i-j) \mathbf{I} \\
& =\sigma_{n}^{2} \sum_{i=1}^{M} \sum_{i=1}^{M} \mathbf{u}_{i} \mathbf{v}_{i}^{H} \mathbf{I} \\
& =\sigma_{n}^{2} \operatorname{Tr}\left(\mathbf{U} \mathbf{V}^{H}\right) \mathbf{I} .
\end{aligned}
$$


APPENDIX B

BIAS DERIVATION FOR EXTREMA SEARCHING ALGORITHMS 
In this section, we will present the detailed derivation of (82)-(86) for MUSIC and derivation of (88)-(92) for Min-Norm. It is recognized that the method used here can be applied to other extrema searching algorithms with slight modification of perturbed weighting matrix. For later convenience, we first introduce some formulas.

Formula 1. Projection Matrix Perturbation

$$
\begin{aligned}
\tilde{\boldsymbol{\Omega}}_{o} & \stackrel{\text { def }}{=} \boldsymbol{\Omega}_{o}+\Delta \boldsymbol{\Omega}_{o} \\
& =\tilde{\mathbf{U}}_{o} \tilde{\mathbf{U}}_{o}^{H} \\
& =\left(\mathbf{U}_{o}+\Delta \mathbf{U}_{o}\right)\left(\mathbf{U}_{o}+\Delta \mathbf{U}_{o}\right)^{H} \\
& =\mathbf{U}_{o} \mathbf{U}_{o}^{H}+\Delta \mathbf{U}_{o} \mathbf{U}_{o}^{H}+\mathbf{U}_{o} \Delta \mathbf{U}_{o}^{H}+\Delta \mathbf{U}_{o} \Delta \mathbf{U}_{o}^{H}
\end{aligned}
$$

Keep $\Delta \boldsymbol{\Omega}_{o}$ to the first-order to obtain

$$
\Delta \boldsymbol{\Omega}_{o \mathbf{1}}=\Delta \mathbf{U}_{o \mathbf{1}} \mathbf{U}_{o}^{H}+\mathbf{U}_{o} \Delta \mathbf{U}_{o \mathbf{1}}^{H} .
$$

Keep $\Delta \boldsymbol{\Omega}_{o}$ to the second-order to obtain

$$
\Delta \boldsymbol{\Omega}_{o 2}=\Delta \mathbf{U}_{o 2} \mathbf{U}_{o}^{H}+\mathbf{U}_{o} \Delta \mathbf{U}_{o 2}^{H}+\Delta \mathbf{U}_{o 1} \Delta \mathbf{U}_{o 1}^{H} .
$$

Formula 2.

$$
\mathbf{U}_{s} \boldsymbol{\Sigma}_{s}^{-2} \mathbf{U}_{s}^{H}=\left(\mathbf{A}^{\dagger}\right)^{H} \hat{\mathbf{R}}_{s}^{-1} \mathbf{A}^{\dagger}
$$

\section{B.1 MUSIC}

For MUSIC,

$$
P\left(\theta_{k}, \tilde{\mathbf{U}}_{o}\right)=\mathbf{a}^{H}\left(\theta_{k}\right) \tilde{\mathbf{\Omega}}_{o} \mathbf{a}\left(\theta_{k}\right) .
$$


By definition (77),

$$
\begin{aligned}
N+\Delta N & \stackrel{\text { def }}{=} \frac{\partial P\left(\theta_{k}, \tilde{\mathbf{U}}_{o}\right)}{\partial \theta} \\
& =\mathbf{a}^{H}\left(\theta_{k}\right) \tilde{\mathbf{\Omega}}_{o} \mathbf{a}^{(1)}\left(\theta_{k}\right)+\mathbf{a}^{(1)}\left(\theta_{k}\right)^{H} \tilde{\mathbf{\Omega}}_{o} \mathbf{a}\left(\theta_{k}\right) \\
& =2 \Re\left\{\mathbf{a}^{H}\left(\theta_{k}\right) \tilde{\mathbf{\Omega}}_{o} \mathbf{a}^{(1)}\left(\theta_{k}\right)\right\} .
\end{aligned}
$$

The zeroth order term is $N$, i.e., without noise perturbation,

$$
N=2 \Re\left\{\mathbf{a}^{H}\left(\theta_{k}\right) \boldsymbol{\Omega}_{o} \mathbf{a}^{(1)}\left(\theta_{k}\right)\right\}
$$

Use (8) to obtain (82). $\Delta N_{1}$ is obtained by approximating (138) to the first-order and throwing away the zeroth order term which is $\mathrm{N}$,

$$
\Delta N_{1}=2 \Re\left\{\mathbf{a}^{H}\left(\theta_{k}\right) \Delta \boldsymbol{\Omega}_{o 1} \mathbf{a}^{(1)}\left(\theta_{k}\right)\right\}
$$

Substitute $\Delta \boldsymbol{\Omega}_{o 1}$ into (139) and use (8) to obtain

$$
\begin{aligned}
\Delta N_{1} & =2 \Re\left\{\mathbf{a}^{H}\left(\theta_{k}\right)\left[\Delta \mathbf{U}_{o 1} \mathbf{U}_{o}^{H}+\mathbf{U}_{o} \Delta \mathbf{U}_{o 1}^{H}\right] \mathbf{a}^{(1)}\left(\theta_{k}\right)\right\} \\
& =2 \Re\left\{\mathbf{a}^{H}\left(\theta_{k}\right) \Delta \mathbf{U}_{o 1} \mathbf{U}_{o}^{H} \mathbf{a}^{(1)}\left(\theta_{k}\right)\right\}
\end{aligned}
$$

and $\Delta N_{2}$ is the second-order term excluding the zeroth order term,

$$
\Delta N_{2}=2 \Re\left\{\mathbf{a}^{H}\left(\theta_{k}\right) \Delta \boldsymbol{\Omega}_{o 2} \mathbf{a}^{(1)}\left(\theta_{k}\right)\right\} .
$$

Substitute $\Delta \boldsymbol{\Omega}_{o 2}$ into (141) and use (8) to obtain

$$
\begin{aligned}
\Delta N_{2} & =2 \Re\left\{\mathbf{a}^{H}\left(\theta_{k}\right)\left[\Delta \mathbf{U}_{o 2} \mathbf{U}_{o}^{H}+\mathbf{U}_{o} \Delta \mathbf{U}_{o 2}^{H}+\Delta \mathbf{U}_{o 1} \Delta \mathbf{U}_{o 1}^{H}\right] \mathbf{a}^{(1)}\left(\theta_{k}\right)\right\} \\
& =2 \Re\left\{\mathbf{a}^{H}\left(\theta_{k}\right) \Delta \mathbf{U}_{o 2} \mathbf{U}_{o}^{H} \mathbf{a}^{(1)}\left(\theta_{k}\right)+\mathbf{a}^{H}\left(\theta_{k}\right) \Delta \mathbf{U}_{o 1} \Delta \mathbf{U}_{o 1}^{H} \mathbf{a}^{(1)}\left(\theta_{k}\right)\right\} .
\end{aligned}
$$

By definition (77),

$$
\begin{aligned}
D+\Delta D & \stackrel{\text { def }}{=} \frac{\partial^{2} P\left(\theta_{k}, \tilde{\mathbf{U}}_{o}\right)}{\partial \theta^{2}} \\
& =\mathbf{a}^{H}\left(\theta_{k}\right) \tilde{\mathbf{\Omega}}_{o} \mathbf{a}^{(2)}\left(\theta_{k}\right)+\mathbf{a}^{(2)}\left(\theta_{k}\right)^{H} \tilde{\mathbf{\Omega}}_{o} \mathbf{a}\left(\theta_{k}\right)+2 \mathbf{a}^{(1)}\left(\theta_{k}\right)^{H} \tilde{\mathbf{\Omega}}_{o} \mathbf{a}^{(1)}\left(\theta_{k}\right) \\
& =2 \Re\left\{\mathbf{a}^{H}\left(\theta_{k}\right) \tilde{\mathbf{\Omega}}_{o} \mathbf{a}^{(2)}\left(\theta_{k}\right)\right\}+2 \mathbf{a}^{(1)}\left(\theta_{k}\right)^{H} \tilde{\mathbf{\Omega}}_{o} \mathbf{a}^{(1)}\left(\theta_{k}\right) .
\end{aligned}
$$


The zeroth order term of $(143)$ is $D$, i.e.,

$$
D=2 \Re\left\{\mathbf{a}^{H}\left(\theta_{k}\right) \boldsymbol{\Omega}_{o} \mathbf{a}^{(2)}\left(\theta_{k}\right)\right\}+2 \mathbf{a}^{(1)}\left(\theta_{k}\right)^{H} \boldsymbol{\Omega}_{o} \mathbf{a}^{(1)}\left(\theta_{k}\right) .
$$

Use (8) to obtain (83). $\Delta D_{1}$ is equals to the residual of the first-order term of (143) as

$$
\Delta D_{1}=2 \Re\left\{\mathbf{a}^{H}\left(\theta_{k}\right) \Delta \boldsymbol{\Omega}_{o 1} \mathbf{a}^{(2)}\left(\theta_{k}\right)\right\}+2 \mathbf{a}^{(1)}\left(\theta_{k}\right)^{H} \Delta \boldsymbol{\Omega}_{o 1} \mathbf{a}^{(1)}\left(\theta_{k}\right)
$$

Substitute $\Delta \boldsymbol{\Omega}_{o 1}$ into (145) and use (8) to obtain

$$
\begin{aligned}
\Delta D_{1}= & 2 \Re\left\{\mathbf{a}^{H}\left(\theta_{k}\right)\left[\Delta \mathbf{U}_{o 1} \mathbf{U}_{o}^{H}+\mathbf{U}_{o} \Delta \mathbf{U}_{o 1}^{H}\right] \mathbf{a}^{(2)}\left(\theta_{k}\right)\right\} \\
& +2 \mathbf{a}^{(1)}\left(\theta_{k}\right)^{H}\left[\Delta \mathbf{U}_{o 1} \mathbf{U}_{o}^{H}+\mathbf{U}_{o} \Delta \mathbf{U}_{o 1}^{H}\right] \mathbf{a}^{(1)}\left(\theta_{k}\right) \\
= & 2 \Re\left\{\mathbf{a}^{H}\left(\theta_{k}\right) \Delta \mathbf{U}_{o 1} \mathbf{U}_{o}^{H} \mathbf{a}^{(2)}\left(\theta_{k}\right)\right\}+4 \Re\left\{\mathbf{a}^{(1)}\left(\theta_{k}\right)^{H} \Delta \mathbf{U}_{o 1} \mathbf{U}_{o}^{H} \mathbf{a}^{(1)}\left(\theta_{k}\right)\right\}
\end{aligned}
$$

By definition (77),

$$
\begin{aligned}
B+\Delta B \stackrel{\text { def }}{=} & \frac{\partial^{3} P\left(\theta_{k}, \tilde{\mathbf{U}}_{o}\right)}{\partial \theta^{3}} \\
= & \mathbf{a}^{H}\left(\theta_{k}\right) \tilde{\mathbf{\Omega}}_{o} \mathbf{a}^{(3)}\left(\theta_{k}\right)+\mathbf{a}^{(3)}\left(\theta_{k}\right)^{H} \tilde{\mathbf{\Omega}}_{o} \mathbf{a}\left(\theta_{k}\right) \\
& 3 \mathbf{a}^{(2)}\left(\theta_{k}\right)^{H} \tilde{\Omega}_{o} \mathbf{a}^{(1)}\left(\theta_{k}\right)+3 \mathbf{a}^{(1)}\left(\theta_{k}\right)^{H} \tilde{\Omega}_{o} \mathbf{a}^{(2)}\left(\theta_{k}\right) .
\end{aligned}
$$

$B$ is the zeroth order term of (147), i.e.,

$$
\begin{aligned}
B= & \mathbf{a}^{H}\left(\theta_{k}\right) \boldsymbol{\Omega}_{o} \mathbf{a}^{(3)}\left(\theta_{k}\right)+\mathbf{a}^{(3)}\left(\theta_{k}\right)^{H} \boldsymbol{\Omega}_{o} \mathbf{a}\left(\theta_{k}\right) \\
& 3 \mathbf{a}^{(2)}\left(\theta_{k}\right)^{H} \boldsymbol{\Omega}_{o} \mathbf{a}^{(1)}\left(\theta_{k}\right)+3 \mathbf{a}^{(1)}\left(\theta_{k}\right)^{H} \boldsymbol{\Omega}_{o} \mathbf{a}^{(2)}\left(\theta_{k}\right) .
\end{aligned}
$$

Use (8) to obtain (84). Substitute (69) into (142) and take expectation on both sides of the equation. Notice $\mathbf{a}^{H}\left(\theta_{k}\right) \mathbf{U}_{o}=0$ so that the expectation of the first term in (142) is zero, we have

$$
E\left(\Delta N_{2}\right)=2 E\left\{\Re\left\{\mathbf{a}^{H}\left(\theta_{k}\right) \Delta \mathbf{U}_{o 1} \Delta \mathbf{U}_{o 1}^{H} \mathbf{a}^{(1)}\left(\theta_{k}\right)\right\}\right\}
$$


Substitute (58), (136) back into the equation

$$
\begin{aligned}
E\left(\Delta N_{2}\right) & =2 \Re\left\{\mathbf{a}^{H}\left(\theta_{k}\right) \mathbf{U}_{s} \Sigma_{s}^{-1} \mathbf{V}_{s}^{H} E\left(\mathbf{N}^{H} \mathbf{U}_{o} \mathbf{U}_{o}^{H} \mathbf{N}\right) \mathbf{V}_{s} \Sigma_{s}^{-1} \mathbf{U}_{s}^{H} \mathbf{a}^{(1)}\left(\theta_{k}\right)\right\} \\
& =2 \Re\left\{\mathbf{a}^{H}\left(\theta_{k}\right) \mathbf{U}_{s} \Sigma_{s}^{-2} \mathbf{U}_{s}^{H} \mathbf{a}^{(1)}\left(\theta_{k}\right)\right\} \operatorname{Tr}\left(\mathbf{U}_{o} \mathbf{U}_{o}^{H}\right) \sigma_{n}^{2} \\
& =2(L-P) \sigma_{n}^{2} \Re\left\{\mathbf{a}^{H}\left(\theta_{k}\right) \mathbf{U}_{s} \Sigma_{s}^{-2} \mathbf{U}_{s}^{H} \mathbf{a}^{(1)}\left(\theta_{k}\right)\right\} .
\end{aligned}
$$

Plug (137) into (148) to obtain (85). Now we begin to derive (86),

$$
\begin{aligned}
E\left(\Delta N_{1} \Delta D_{1}\right)= & 4 E\left\{\Re\left\{\mathbf{a}^{H}\left(\theta_{k}\right) \Delta \mathbf{U}_{o 1} \mathbf{U}_{o}^{H} \mathbf{a}^{(1)}\left(\theta_{k}\right)\right\} \Re\left\{\mathbf{a}^{H}\left(\theta_{k}\right) \Delta \mathbf{U}_{o 1} \mathbf{U}_{o}^{H} \mathbf{a}^{(2)}\left(\theta_{k}\right)\right\}\right\} \\
& +8 E\left\{\Re\left\{\mathbf{a}^{H}\left(\theta_{k}\right) \Delta \mathbf{U}_{o 1} \mathbf{U}_{o}^{H} \mathbf{a}^{(1)}\left(\theta_{k}\right)\right\} \Re\left\{\mathbf{a}^{(1)}\left(\theta_{k}\right)^{H} \Delta \mathbf{U}_{o 1} \mathbf{U}_{o}^{H} \mathbf{a}^{(1)}\left(\theta_{k}\right)\right\}\right\}
\end{aligned}
$$

Since $\Delta \mathbf{U}_{o 1}$ is linear to $\mathbf{N}, E\left(\Delta \mathbf{U}_{o 1} \Delta \mathbf{U}_{o 1}\right)=0$ because of the circularity of the noise. Thus (149) can be reduced to

$$
\begin{aligned}
E\left(\Delta N_{1} \Delta D_{1}\right)= & 2 E\left\{\Re\left\{\mathbf{a}^{H}\left(\theta_{k}\right) \Delta \mathbf{U}_{o 1} \mathbf{U}_{o}^{H} \mathbf{a}^{(1)}\left(\theta_{k}\right) \mathbf{a}^{(2)}\left(\theta_{k}\right)^{H} \mathbf{U}_{o} \Delta \mathbf{U}_{o 1}^{H} \mathbf{a}\left(\theta_{k}\right)\right\}\right\} \\
& +4 E\left\{\Re\left\{\mathbf{a}^{H}\left(\theta_{k}\right) \Delta \mathbf{U}_{o 1} \mathbf{U}_{o}^{H} \mathbf{a}^{(1)}\left(\theta_{k}\right) \mathbf{a}^{(1)}\left(\theta_{k}\right)^{H} \mathbf{U}_{o} \Delta \mathbf{U}_{o 1}^{H} \mathbf{a}^{(1)}\left(\theta_{k}\right)\right\}\right\}
\end{aligned}
$$

Substitute (58), (136) back into the equation

$$
\begin{aligned}
& E\left(\Delta N_{1} \Delta D_{1}\right) \\
= & 2 E\left\{\Re\left\{\mathbf{a}^{H}\left(\theta_{k}\right) \mathbf{U}_{s} \Sigma_{s}^{-1} \mathbf{V}_{s}^{H} \mathbf{N}^{H} \mathbf{U}_{o} \mathbf{U}_{o}^{H} \mathbf{a}^{(1)}\left(\theta_{k}\right) \mathbf{a}^{(2)}\left(\theta_{k}\right)^{H} \mathbf{U}_{o} \mathbf{U}_{o}^{H} \mathbf{N V}_{s} \Sigma_{s}^{-1} \mathbf{U}_{s}^{H} \mathbf{a}\left(\theta_{k}\right)\right\}\right\} \\
& +4 E\left\{\Re\left\{\mathbf{a}^{H}\left(\theta_{k}\right) \mathbf{U}_{s} \Sigma_{s}^{-1} \mathbf{V}_{s}^{H} \mathbf{N}^{H} \mathbf{U}_{o} \mathbf{U}_{o}^{H} \mathbf{a}^{(1)}\left(\theta_{k}\right) \mathbf{a}^{(1)}\left(\theta_{k}\right)^{H} \mathbf{U}_{o} \mathbf{U}_{o}^{H} \mathbf{N V}_{s} \Sigma_{s}^{-1} \mathbf{U}_{s}^{H} \mathbf{a}^{(1)}\left(\theta_{k}\right)\right\}\right\} \\
= & 2 \sigma_{n}^{2} \Re\left\{\mathbf{a}^{H}\left(\theta_{k}\right) \mathbf{U}_{s} \Sigma_{s}^{-1} \mathbf{V}_{s}^{H} \mathbf{V}_{s} \Sigma_{s}^{-1} \mathbf{U}_{s}^{H} \mathbf{a}\left(\theta_{k}\right) \operatorname{Tr}\left(\mathbf{U}_{o} \mathbf{U}_{o}^{H} \mathbf{a}^{(1)}\left(\theta_{k}\right) \mathbf{a}^{(2)}\left(\theta_{k}\right)^{H} \mathbf{U}_{o} \mathbf{U}_{o}^{H}\right)\right\} \\
& +4 \sigma_{n}^{2} \Re\left\{\mathbf{a}^{H}\left(\theta_{k}\right) \mathbf{U}_{s} \Sigma_{s}^{-1} \mathbf{V}_{s}^{H} \mathbf{V}_{s} \Sigma_{s}^{-1} \mathbf{U}_{s}^{H} \mathbf{a}^{(1)}\left(\theta_{k}\right) \operatorname{Tr}\left(\mathbf{U}_{o} \mathbf{U}_{o}^{H} \mathbf{a}^{(1)}\left(\theta_{k}\right) \mathbf{a}^{(1)}\left(\theta_{k}\right)^{H} \mathbf{U}_{o} \mathbf{U}_{o}^{H}\right)\right\} \\
= & 2 \sigma_{n}^{2} \Re\left\{\mathbf{a}^{H}\left(\theta_{k}\right) \mathbf{U}_{s} \Sigma_{s}^{-2} \mathbf{U}_{s}^{H} \mathbf{a}\left(\theta_{k}\right) \mathbf{a}^{(2)}\left(\theta_{k}\right)^{H} \Omega_{o} \mathbf{a}^{(1)}\left(\theta_{k}\right)\right\} \\
& +4 \sigma_{n}^{2} \Re\left\{\mathbf{a}^{H}\left(\theta_{k}\right) \mathbf{U}_{s} \Sigma_{s}^{-2} \mathbf{U}_{s}^{H} \mathbf{a}^{(1)}\left(\theta_{k}\right) \mathbf{a}^{(1)}\left(\theta_{k}\right)^{H} \Omega_{o} \mathbf{a}^{(1)}\left(\theta_{k}\right)\right\} .
\end{aligned}
$$


Plug (137) into (150) to obtain (86).

\section{B.2 MIN-NORM}

For Min-Norm,

$$
\begin{aligned}
P\left(\theta_{k}, \tilde{\mathbf{U}}_{o}\right) & =\mathbf{a}^{H}\left(\theta_{k}\right) \tilde{\mathbf{U}}_{o} \overline{\mathbf{c}} \overline{\mathbf{c}}^{H} \tilde{\mathbf{U}}_{o}^{H} \mathbf{a}\left(\theta_{k}\right) \\
& =\frac{1}{\left\|\mathbf{e}_{1}^{H} \tilde{\mathbf{\Omega}}_{o}\right\|^{4}} \mathbf{a}^{H}\left(\theta_{k}\right) \tilde{\mathbf{\Omega}}_{o} \mathbf{e}_{1} \mathbf{e}_{1}^{H} \tilde{\mathbf{\Omega}}_{o} \mathbf{a}\left(\theta_{k}\right)
\end{aligned}
$$

By definition (77),

$$
\begin{aligned}
N+\Delta N & \stackrel{\text { def }}{=} \frac{\partial P\left(\theta_{k}, \tilde{\mathbf{U}}_{o}\right)}{\partial \theta} \\
& =\frac{1}{\left\|\mathbf{e}_{1}^{H} \tilde{\mathbf{\Omega}}_{o}\right\|^{4}}\left\{\mathbf{a}^{(1)}\left(\theta_{k}\right)^{H} \tilde{\mathbf{\Omega}}_{o} \mathbf{e}_{1} \mathbf{e}_{1}^{H} \tilde{\mathbf{\Omega}}_{o} \mathbf{a}\left(\theta_{k}\right)+\mathbf{a}^{H}\left(\theta_{k}\right) \tilde{\mathbf{\Omega}}_{o} \mathbf{e}_{1} \mathbf{e}_{1}^{H} \tilde{\mathbf{\Omega}}_{o} \mathbf{a}^{(1)}\left(\theta_{k}\right)\right\} \\
& =\frac{2}{\left\|\mathbf{e}_{1}^{H} \tilde{\mathbf{\Omega}}_{o}\right\|^{4}} \Re\left\{\mathbf{a}^{(1)}\left(\theta_{k}\right)^{H} \tilde{\mathbf{\Omega}}_{o} \mathbf{e}_{1} \mathbf{e}_{1}^{H} \tilde{\mathbf{\Omega}}_{o} \mathbf{a}\left(\theta_{k}\right)\right\}
\end{aligned}
$$

The zeroth order term is $\mathrm{N}$, i.e.,

$$
N=\frac{2}{\left\|\mathbf{e}_{1}^{H} \boldsymbol{\Omega}_{o}\right\|^{4}} \Re\left\{\mathbf{a}^{(1)}\left(\theta_{k}\right)^{H} \boldsymbol{\Omega}_{o} \mathbf{e}_{1} \mathbf{e}_{1}^{H} \boldsymbol{\Omega}_{o} \mathbf{a}\left(\theta_{k}\right)\right\}
$$

Use (8) to obtain (88). Keep (151) up to the first-order and throw away the zeroth order term to obtain $\Delta N_{1}$,

$$
\Delta N_{1}=\frac{2}{\left\|\mathbf{e}_{1}^{H} \boldsymbol{\Omega}_{o}\right\|^{4}} \Re\left\{\mathbf{a}^{(1)}\left(\theta_{k}\right)^{H}\left[\boldsymbol{\Omega}_{o} \mathbf{e}_{1} \mathbf{e}_{1}^{H} \Delta \boldsymbol{\Omega}_{o 1}+\Delta \boldsymbol{\Omega}_{o 1} \mathbf{e}_{1} \mathbf{e}_{1}^{H} \boldsymbol{\Omega}_{o}\right] \mathbf{a}\left(\theta_{k}\right)\right\}
$$

Substitute $\Delta \boldsymbol{\Omega}_{01}$ into (153) and use (8) to obtain

$$
\begin{aligned}
\Delta N_{1} & =\frac{2}{\left\|\mathbf{e}_{1}^{H} \boldsymbol{\Omega}_{o}\right\|^{4}} \Re\left\{\mathbf{a}^{(1)}\left(\theta_{k}\right)^{H} \boldsymbol{\Omega}_{o} \mathbf{e}_{1} \mathbf{e}_{1}^{H} \Delta \boldsymbol{\Omega}_{o 1} \mathbf{a}\left(\theta_{k}\right)\right\} \\
& =\frac{2}{\left\|\mathbf{e}_{1}^{H} \boldsymbol{\Omega}_{o}\right\|^{4}} \Re\left\{\mathbf{a}^{(1)}\left(\theta_{k}\right)^{H} \boldsymbol{\Omega}_{o} \mathbf{e}_{1} \mathbf{e}_{1}^{H}\left[\Delta \mathbf{U}_{o 1} \mathbf{U}_{o}^{H}+\mathbf{U}_{o} \Delta \mathbf{U}_{o 1}^{H}\right] \mathbf{a}\left(\theta_{k}\right)\right\} \\
& =\frac{2}{\left\|\mathbf{e}_{1}^{H} \boldsymbol{\Omega}_{o}\right\|^{4}} \Re\left\{\mathbf{a}^{(1)}\left(\theta_{k}\right)^{H} \Omega_{o} \mathbf{e}_{1} \mathbf{e}_{1}^{H} \mathbf{U}_{o} \Delta \mathbf{U}_{o 1}^{H} \mathbf{a}\left(\theta_{k}\right)\right\}
\end{aligned}
$$


Keep (151) up to the second-order and exclude the zeroth order term to obtain $\Delta N_{2}$

$$
\Delta N_{2}=\frac{2}{\left\|\mathbf{e}_{1}^{H} \boldsymbol{\Omega}_{o}\right\|^{4}} \Re\left\{\mathbf{a}^{(1)}\left(\theta_{k}\right)^{H}\left[\boldsymbol{\Omega}_{o} \mathbf{e}_{1} \mathbf{e}_{1}^{H} \Delta \boldsymbol{\Omega}_{o 2}+\Delta \boldsymbol{\Omega}_{o 2} \mathbf{e}_{1} \mathbf{e}_{1}^{H} \boldsymbol{\Omega}_{o}+\Delta \boldsymbol{\Omega}_{o 1} \mathbf{e}_{1} \mathbf{e}_{1}^{H} \Delta \boldsymbol{\Omega}_{o 1}\right] \mathbf{a}\left(\theta_{k}\right)\right\}
$$

Substitute $\Delta \boldsymbol{\Omega}_{o 1}$ and $\Delta \boldsymbol{\Omega}_{\circ 2}$ into (155) and use (8) to obtain

$$
\begin{aligned}
& \Delta N_{2}=\frac{2}{\left\|\mathbf{e}_{1}^{H} \boldsymbol{\Omega}_{o}\right\|^{4}} \Re\left\{\mathbf{a}^{(1)}\left(\theta_{k}\right)^{H}\left[\boldsymbol{\Omega}_{o} \mathbf{e}_{1} \mathbf{e}_{1}^{H} \Delta \boldsymbol{\Omega}_{o 2}+\Delta \boldsymbol{\Omega}_{o 1} \mathbf{e}_{1} \mathbf{e}_{1}^{H} \Delta \boldsymbol{\Omega}_{o 1}\right] \mathbf{a}\left(\theta_{k}\right)\right\} \\
& =\frac{2}{\left\|\mathbf{e}_{1}^{H} \Omega_{o}\right\|^{4}} \Re\left\{\mathbf{a}^{(1)}\left(\theta_{k}\right)^{H} \Omega_{o} \mathbf{e}_{1} \mathbf{e}_{1}^{H}\left[\Delta \mathrm{U}_{o 2} \mathbf{U}_{o}^{H}+\mathrm{U}_{o} \Delta \mathrm{U}_{o 2}^{H}+\Delta \mathrm{U}_{o 1} \Delta \mathrm{U}_{o 1}^{H}\right] \mathrm{a}\left(\theta_{k}\right)\right. \\
& \left.+\mathbf{a}^{(1)}\left(\theta_{k}\right)^{H}\left[\Delta \mathbf{U}_{o 1} \mathbf{U}_{o}^{H}+\mathrm{U}_{o} \Delta \mathrm{U}_{o 1}^{H}\right] \mathbf{e}_{1} \mathbf{e}_{1}^{H}\left[\Delta \mathrm{U}_{o 1} \mathrm{U}_{o}^{H}+\mathrm{U}_{o} \Delta \mathbf{U}_{o 1}^{H}\right] \mathbf{a}\left(\theta_{k}\right)\right\} \\
& =\frac{2}{\left\|\mathbf{e}_{1}^{H} \Omega_{o}\right\|^{4}} \Re\left\{\mathbf{a}^{(1)}\left(\theta_{k}\right)^{H} \boldsymbol{\Omega}_{o} \mathbf{e}_{1} \mathbf{e}_{1}^{H}\left[\mathbf{U}_{o} \Delta \mathbf{U}_{o 2}^{H}+\Delta \mathbf{U}_{o 1} \Delta \mathbf{U}_{o 1}^{H}\right] \mathbf{a}\left(\theta_{k}\right)\right. \\
& \left.+\mathbf{a}^{(1)}\left(\theta_{k}\right)^{H}\left[\Delta \mathbf{U}_{o 1} \mathbf{U}_{o}^{H}+\mathbf{U}_{o} \Delta \mathbf{U}_{o 1}^{H}\right] \mathbf{e}_{1} \mathbf{e}_{1}^{H} \mathbf{U}_{o} \Delta \mathbf{U}_{o 1}^{H} \mathbf{a}\left(\theta_{k}\right)\right\}
\end{aligned}
$$

By definition (77),

$$
\begin{aligned}
D+\Delta D \stackrel{\text { def }}{=} & \frac{\partial^{2} P\left(\theta_{k}, \tilde{\mathbf{U}}_{o}\right)}{\partial \theta^{2}} \\
= & \frac{1}{\left\|\mathbf{e}_{1}^{H} \tilde{\mathbf{\Omega}}_{o}\right\|^{4}}\left\{\mathbf{a}^{(2)}\left(\theta_{k}\right)^{H} \tilde{\mathbf{\Omega}}_{o} \mathbf{e}_{1} \mathbf{e}_{1}^{H} \tilde{\mathbf{\Omega}}_{o} \mathbf{a}\left(\theta_{k}\right)\right. \\
& \left.+2 \mathbf{a}^{(1)}\left(\theta_{k}\right)^{H} \tilde{\mathbf{\Omega}}_{o} \mathbf{e}_{1} \mathbf{e}_{1}^{H} \tilde{\mathbf{\Omega}}_{o} \mathbf{a}^{(1)}\left(\theta_{k}\right)+\mathbf{a}\left(\theta_{k}\right)^{H} \tilde{\mathbf{\Omega}}_{o} \mathbf{e}_{1} \mathbf{e}_{1}^{H} \tilde{\mathbf{\Omega}}_{o} \mathbf{a}^{(2)}\left(\theta_{k}\right)\right\} \\
= & \frac{1}{\left\|\mathbf{e}_{1}^{H} \tilde{\mathbf{\Omega}}_{o}\right\|^{4}}\left\{2 \Re\left\{\mathbf{a}^{(2)}\left(\theta_{k}\right)^{H} \tilde{\mathbf{\Omega}}_{o} \mathbf{e}_{1} \mathbf{e}_{1}^{H} \tilde{\Omega}_{o} \mathbf{a}\left(\theta_{k}\right)\right\}\right. \\
& \left.+2 \mathbf{a}^{(1)}\left(\theta_{k}\right)^{H} \tilde{\Omega}_{o} \mathbf{e}_{1} \mathbf{e}_{1}^{H} \tilde{\Omega}_{o} \mathbf{a}^{(1)}\left(\theta_{k}\right)\right\}
\end{aligned}
$$

The zeroth order term is $D$, i.e.,

$$
D=\frac{1}{\left\|\mathbf{e}_{1}^{H} \boldsymbol{\Omega}_{o}\right\|^{4}}\left\{2 \Re\left\{\mathbf{a}^{(2)}\left(\theta_{k}\right)^{H} \boldsymbol{\Omega}_{o} \mathbf{e}_{1} \mathbf{e}_{1}^{H} \boldsymbol{\Omega}_{o} \mathbf{a}\left(\theta_{k}\right)\right\}+2 \mathbf{a}^{(1)}\left(\theta_{k}\right)^{H} \boldsymbol{\Omega}_{o} \mathbf{e}_{1} \mathbf{e}_{1}^{H} \boldsymbol{\Omega}_{o} \mathbf{a}^{(1)}\left(\theta_{k}\right)\right\}
$$

Use (8) to obtain (89). Keep (157) up to the first-order and throw away the zeroth order term to obtain $\Delta D_{1}$

$$
\begin{aligned}
\Delta D_{1}= & \frac{1}{\left\|\mathbf{e}_{1}^{H} \boldsymbol{\Omega}_{o}\right\|^{4}}\left\{2 \Re\left\{\mathbf{a}^{(2)}\left(\theta_{k}\right)^{H}\left[\Delta \boldsymbol{\Omega}_{o 1} \mathbf{e}_{1} \mathbf{e}_{1}^{H} \boldsymbol{\Omega}_{o}+\boldsymbol{\Omega}_{o} \mathbf{e}_{1} \mathbf{e}_{1}^{H} \Delta \boldsymbol{\Omega}_{o 1}\right] \mathbf{a}\left(\theta_{k}\right)\right\}\right. \\
& \left.+2 \mathbf{a}^{(1)}\left(\theta_{k}\right)^{H}\left[\Delta \boldsymbol{\Omega}_{o 1} \mathbf{e}_{1} \mathbf{e}_{1}^{H} \boldsymbol{\Omega}_{o}+\boldsymbol{\Omega}_{o} \mathbf{e}_{1} \mathbf{e}_{1}^{H} \Delta \boldsymbol{\Omega}_{o 1}\right] \mathbf{a}^{(1)}\left(\theta_{k}\right)\right\}
\end{aligned}
$$


Substitute $\Delta \boldsymbol{\Omega}_{o 1}$ into (158) and use (8) to obtain

$$
\begin{aligned}
\Delta D_{1}= & \frac{1}{\left\|\mathbf{e}_{1}^{H} \boldsymbol{\Omega}_{o}\right\|^{4}}\left\{2 \Re\left\{\mathbf{a}^{(2)}\left(\theta_{k}\right)^{H} \boldsymbol{\Omega}_{o} \mathbf{e}_{1} \mathbf{e}_{1}^{H} \Delta \boldsymbol{\Omega}_{o 1} \mathbf{a}\left(\theta_{k}\right)\right\}\right. \\
& \left.+4 \Re\left\{\mathbf{a}^{(1)}\left(\theta_{k}\right)^{H} \Delta \boldsymbol{\Omega}_{o 1} \mathbf{e}_{1} \mathbf{e}_{1}^{H} \boldsymbol{\Omega}_{o} \mathbf{a}^{(1)}\left(\theta_{k}\right)\right\}\right\} \\
= & \frac{1}{\left\|\mathbf{e}_{1}^{H} \boldsymbol{\Omega}_{o}\right\|^{4}}\left\{2 \Re\left\{\mathbf{a}^{(2)}\left(\theta_{k}\right)^{H} \boldsymbol{\Omega}_{o} \mathbf{e}_{1} \mathbf{e}_{1}^{H}\left[\Delta \mathbf{U}_{o 1} \mathbf{U}_{o}^{H}+\mathbf{U}_{o} \Delta \mathbf{U}_{o 1}^{H}\right] \mathbf{a}\left(\theta_{k}\right)\right\}\right. \\
& \left.+4 \Re\left\{\mathbf{a}^{(1)}\left(\theta_{k}\right)^{H}\left[\Delta \mathbf{U}_{o 1} \mathbf{U}_{o}^{H}+\mathbf{U}_{o} \Delta \mathbf{U}_{o 1}^{H}\right] \mathbf{e}_{1} \mathbf{e}_{1}^{H} \boldsymbol{\Omega}_{o} \mathbf{a}^{(1)}\left(\theta_{k}\right)\right\}\right\} \\
= & \frac{1}{\left\|\mathbf{e}_{1}^{H} \boldsymbol{\Omega}_{o}\right\|^{4}}\left\{2 \Re\left\{\mathbf{a}^{(2)}\left(\theta_{k}\right)^{H} \boldsymbol{\Omega}_{o} \mathbf{e}_{1} \mathbf{e}_{1}^{H} \mathbf{U}_{o} \Delta \mathbf{U}_{o 1}^{H} \mathbf{a}\left(\theta_{k}\right)\right\}\right. \\
& +4 \Re\left\{\mathbf{a}^{(1)}\left(\theta_{k}\right)^{H} \Delta \mathbf{U}_{o 1} \mathbf{U}_{o}^{H} \mathbf{e}_{1} \mathbf{e}_{1}^{H} \boldsymbol{\Omega}_{o} \mathbf{a}^{(1)}\left(\theta_{k}\right)\right\} \\
& \left.+4 \Re\left\{\mathbf{a}^{(1)}\left(\theta_{k}\right)^{H} \mathbf{U}_{o} \Delta \mathbf{U}_{o 1}^{H} \mathbf{e}_{1} \mathbf{e}_{1}^{H} \boldsymbol{\Omega}_{o} \mathbf{a}^{(1)}\left(\theta_{k}\right)\right\}\right\} .
\end{aligned}
$$

By definition (77),

$$
\begin{aligned}
B+\Delta B \stackrel{\text { def }}{=} & \frac{\partial^{3} P\left(\theta_{k}, \tilde{\mathbf{U}}_{o}\right)}{\partial \theta^{3}} \\
= & \frac{1}{\left\|\mathbf{e}_{1}^{H} \tilde{\mathbf{\Omega}}_{o}\right\|^{4}}\left\{\mathbf{a}^{(3)}\left(\theta_{k}\right)^{H} \tilde{\mathbf{\Omega}}_{o} \mathbf{e}_{1} \mathbf{e}_{1}^{H} \tilde{\mathbf{\Omega}}_{o} \mathbf{a}\left(\theta_{k}\right)+\mathbf{a}^{H}\left(\theta_{k}\right) \tilde{\Omega}_{o} \mathbf{e}_{1} \mathbf{e}_{1}^{H} \tilde{\mathbf{\Omega}}_{o} \mathbf{a}^{(3)}\left(\theta_{k}\right)\right. \\
& \left.+3 \mathbf{a}^{(2)}\left(\theta_{k}\right)^{H} \tilde{\mathbf{\Omega}}_{o} \mathbf{e}_{1} \mathbf{e}_{1}^{H} \tilde{\mathbf{\Omega}}_{o} \mathbf{a}^{(1)}\left(\theta_{k}\right)+3 \mathbf{a}^{(1)}\left(\theta_{k}\right)^{H} \tilde{\mathbf{\Omega}}_{o} \mathbf{e}_{1} \mathbf{e}_{1}^{H} \tilde{\Omega}_{o} \mathbf{a}^{(2)}\left(\theta_{k}\right)\right\}
\end{aligned}
$$

Keep the zeroth order term to obtain B, i.e.,

$$
B=\frac{1}{\left\|\mathbf{e}_{1}^{H} \boldsymbol{\Omega}_{o}\right\|^{4}}\left\{2 \Re\left\{\mathbf{a}^{(3)}\left(\theta_{k}\right)^{H} \boldsymbol{\Omega}_{o} \mathbf{e}_{1} \mathbf{e}_{1}^{H} \boldsymbol{\Omega}_{o} \mathbf{a}\left(\theta_{k}\right)\right\}+6 \Re\left\{\mathbf{a}^{(2)}\left(\theta_{k}\right)^{H} \boldsymbol{\Omega}_{o} \mathbf{e}_{1} \mathbf{e}_{1}^{H} \boldsymbol{\Omega}_{o} \mathbf{a}^{(1)}\left(\theta_{k}\right)\right\}\right\} .
$$

Using (8) to obtain (90). Substitute (69) into (156) and take expectation on both sides of the equation. Using $\mathbf{a}^{H}\left(\theta_{k}\right) \mathbf{U}_{o}=0$ as well as the circularity of the noise, we have

$$
\begin{aligned}
E\left(\Delta N_{2}\right)= & \frac{2}{\left\|\mathbf{e}_{1}^{H} \Omega_{o}\right\|^{4}} \Re\left\{E\left[\mathbf{a}^{(1)}\left(\theta_{k}\right)^{H} \Omega_{o} \mathbf{e}_{1} \mathbf{e}_{1}^{H} \Delta \mathbf{U}_{o 1} \Delta \mathbf{U}_{o 1}^{H} \mathbf{a}\left(\theta_{k}\right)\right]\right. \\
& \left.+E\left[\mathbf{a}^{(1)}\left(\theta_{k}\right)^{H} \Delta \mathbf{U}_{o 1} \mathbf{U}_{o}^{H} \mathbf{e}_{1} \mathbf{e}_{1}^{H} \mathbf{U}_{o} \Delta \mathbf{U}_{o 1}^{H} \mathbf{a}\left(\theta_{k}\right)\right]\right\}
\end{aligned}
$$

Substitute (58) and (136) back into the equation

$$
E\left(\Delta N_{2}\right)=\frac{2}{\left\|\mathbf{e}_{1}^{H} \Omega_{o}\right\|^{4}} \Re\left\{E\left[\mathbf{a}^{(1)}\left(\theta_{k}\right)^{H} \Omega_{o} \mathbf{e}_{1} \mathbf{e}_{1}^{H} \mathbf{U}_{s} \boldsymbol{\Sigma}_{s}^{-1} \mathbf{V}_{s}^{H} \mathbf{N}^{H} \mathbf{U}_{o} \mathbf{U}_{o}^{H} \mathbf{N} \mathbf{V}_{s} \boldsymbol{\Sigma}_{s}^{-1} \mathbf{U}_{s}^{H} \mathbf{a}\left(\theta_{k}\right)\right]\right.
$$




$$
\begin{aligned}
& \left.+E\left[\mathbf{a}^{(1)}\left(\theta_{k}\right)^{H} \mathbf{U}_{s} \Sigma_{s}^{-1} \mathbf{V}_{s}^{H} \mathbf{N}^{H} \mathbf{U}_{o} \mathbf{U}_{o}^{H} \mathbf{e}_{1} \mathbf{e}_{1}^{H} \mathbf{U}_{o} \mathbf{U}_{o}^{H} \mathbf{N V}_{s} \Sigma_{s}^{-1} \mathbf{U}_{s}^{H} \mathbf{a}\left(\theta_{k}\right)\right]\right\} \\
= & \frac{2 \sigma_{n}^{2}}{\left\|\mathbf{e}_{1}^{H} \Omega_{o}\right\|^{4}} \Re\left\{\mathbf{a}^{(1)}\left(\theta_{k}\right)^{H} \Omega_{o} \mathbf{e}_{1} \mathbf{e}_{1}^{H} \mathbf{U}_{s} \Sigma_{s}^{-1} \mathbf{V}_{s}^{H} \mathbf{V}_{s} \Sigma_{s}^{-1} \mathbf{U}_{s}^{H} \mathbf{a}\left(\theta_{k}\right) \operatorname{Tr}\left(\mathbf{U}_{o} \mathbf{U}_{o}^{H}\right)\right. \\
& \left.+\mathbf{a}^{(1)}\left(\theta_{k}\right)^{H} \mathbf{U}_{s} \Sigma_{s}^{-1} \mathbf{V}_{s}^{H} \mathbf{V}_{s} \Sigma_{s}^{-1} \mathbf{U}_{s}^{H} \mathbf{a}\left(\theta_{k}\right) \operatorname{Tr}\left(\mathbf{U}_{o} \mathbf{U}_{o}^{H} \mathbf{e}_{1} \mathbf{e}_{1}^{H} \mathbf{U}_{o} \mathbf{U}_{o}^{H}\right)\right\} \\
= & \frac{2 \sigma_{n}^{2}}{\left\|\mathbf{e}_{1}^{H} \boldsymbol{\Omega}_{o}\right\|^{4}} \Re\left\{(L-P) \mathbf{a}^{(1)}\left(\theta_{k}\right)^{H} \Omega_{o} \mathbf{e}_{1} \mathbf{e}_{1}^{H} \mathbf{U}_{s} \boldsymbol{\Sigma}_{s}^{-2} \mathbf{U}_{s}^{H} \mathbf{a}\left(\theta_{k}\right)\right. \\
& \left.+\left\|\mathbf{e}_{1} \Omega_{o}\right\|^{2} \mathbf{a}^{(1)}\left(\theta_{k}\right)^{H} \mathbf{U}_{s} \Sigma_{s}^{-2} \mathbf{U}_{s}^{H} \mathbf{a}\left(\theta_{k}\right)\right\} .
\end{aligned}
$$

Plug (137) into (161) to obtain (91). Now let us calculate $E\left(\Delta N_{1} \Delta D_{1}\right)$ by using (154) and (159),

$$
\begin{aligned}
& E\left(\Delta N_{1} \Delta D_{1}\right) \\
= & \frac{4}{\left\|\mathbf{e}_{1}^{H} \boldsymbol{\Omega}_{o}\right\|^{8}}\left\{E \left\{\Re\left\{\mathbf{a}^{(1)}\left(\theta_{k}\right)^{H} \boldsymbol{\Omega}_{o} \mathbf{e}_{1} \mathbf{e}_{1}^{H} \mathbf{U}_{o} \Delta \mathbf{U}_{o 1}^{H} \mathbf{a}\left(\theta_{k}\right)\right\}\right.\right. \\
& \left.\Re\left\{\mathbf{a}^{(2)}\left(\theta_{k}\right)^{H} \boldsymbol{\Omega}_{o} \mathbf{e}_{1} \mathbf{e}_{1}^{H} \mathbf{U}_{o} \Delta \mathbf{U}_{o 1}^{H} \mathbf{a}\left(\theta_{k}\right)\right\}\right\}+2 E\left\{\Re\left\{\mathbf{a}^{(1)}\left(\theta_{k}\right)^{H} \boldsymbol{\Omega}_{o} \mathbf{e}_{1} \mathbf{e}_{1}^{H} \mathbf{U}_{o} \Delta \mathbf{U}_{o 1}^{H} \mathbf{a}\left(\theta_{k}\right)\right\}\right. \\
& \left.\Re\left\{\mathbf{a}^{(1)}\left(\theta_{k}\right)^{H} \Delta \mathbf{U}_{o 1} \mathbf{U}_{o}^{H} \mathbf{e}_{1} \mathbf{e}_{1}^{H} \boldsymbol{\Omega}_{o} \mathbf{a}^{(1)}\left(\theta_{k}\right)\right\}\right\}+2 E\left\{\Re\left\{\mathbf{a}^{(1)}\left(\theta_{k}\right)^{H} \boldsymbol{\Omega}_{o} \mathbf{e}_{1} \mathbf{e}_{1}^{H} \mathbf{U}_{o} \Delta \mathbf{U}_{o 1}^{H} \mathbf{a}\left(\theta_{k}\right)\right\}\right. \\
& \left.\Re\left\{\mathbf{a}^{(1)}\left(\theta_{k}\right)^{H} \mathbf{U}_{o} \Delta \mathbf{U}_{o 1}^{H} \mathbf{e}_{1} \mathbf{e}_{1}^{H} \boldsymbol{\Omega}_{o} \mathbf{a}^{(1)}\left(\theta_{k}\right)\right\}\right\} .
\end{aligned}
$$

Use the circular property of the noise to simplify

$$
\begin{aligned}
& E\left(\Delta N_{1} \Delta D_{1}\right) \\
= & \frac{2}{\left\|\mathbf{e}_{1}^{H} \boldsymbol{\Omega}_{o}\right\|^{8}}\left\{\Re\left\{\mathbf{a}^{(1)}\left(\theta_{k}\right)^{H} \Omega_{o} \mathbf{e}_{1} \mathbf{e}_{1}^{H} \mathbf{U}_{o} \Delta \mathbf{U}_{o 1}^{H} \mathbf{a}\left(\theta_{k}\right) \mathbf{a}^{H}\left(\theta_{k}\right) \Delta \mathbf{U}_{o 1} \mathbf{U}_{o}^{H} \mathbf{e}_{1} \mathbf{e}_{1}^{H} \boldsymbol{\Omega}_{o} \mathbf{a}^{(2)}\left(\theta_{k}\right)\right\}\right. \\
& \left.+2 \Re\left\{\mathbf{a}^{(1)}\left(\theta_{k}\right)^{H} \Omega_{o} \mathbf{e}_{1} \mathbf{e}_{1}^{H} \mathbf{U}_{o} \Delta \mathbf{U}_{o 1}^{H} \mathbf{a}\left(\theta_{k}\right)\right\} \mathbf{a}^{(1)}\left(\theta_{k}\right)^{H} \Delta \mathbf{U}_{o 1} \mathbf{U}_{o}^{H} \mathbf{e}_{1} \mathbf{e}_{1}^{H} \boldsymbol{\Omega}_{o} \mathbf{a}^{(1)}\left(\theta_{k}\right)\right\} \\
& \left.+2 \Re\left\{\mathbf{a}^{(1)}\left(\theta_{k}\right)^{H} \boldsymbol{\Omega}_{o} \mathbf{e}_{1} \mathbf{e}_{1}^{H} \mathbf{U}_{o} \Delta \mathbf{U}_{o 1}^{H} \mathbf{a}\left(\theta_{k}\right) \mathbf{a}^{(1)}\left(\theta_{k}\right)^{H} \boldsymbol{\Omega}_{o} \mathbf{e}_{1} \mathbf{e}_{1}^{H} \Delta \mathbf{U}_{o 1} \mathbf{U}_{o}^{H} \mathbf{a}^{(1)}\left(\theta_{k}\right)\right\}\right\} .
\end{aligned}
$$

Substitute (58) and (136) back into the equation

$$
\begin{aligned}
& E\left(\Delta N_{1} \Delta D_{1}\right) \\
= & \frac{2}{\left\|\mathbf{e}_{1}^{H} \boldsymbol{\Omega}_{o}\right\|^{8}}\left\{\Re \left\{\mathbf{a}^{(1)}\left(\theta_{k}\right)^{H} \boldsymbol{\Omega}_{o} \mathbf{e}_{1} \mathbf{e}_{1}^{H} \mathbf{U}_{o} \mathbf{U}_{o}^{H} \mathbf{N} \mathbf{V}_{s} \boldsymbol{\Sigma}_{s}^{-1} \mathbf{U}_{s}^{H} \mathbf{a}\left(\theta_{k}\right)\right.\right. \\
& \left.\mathbf{a}^{H}\left(\theta_{k}\right) \mathbf{U}_{s} \Sigma_{s}^{-1} \mathbf{V}_{s}^{H} \mathbf{N}^{H} \mathbf{U}_{o} \mathbf{U}_{o}^{H} \mathbf{e}_{1} \mathbf{e}_{1}^{H} \boldsymbol{\Omega}_{o} \mathbf{a}^{(2)}\left(\theta_{k}\right)\right\}
\end{aligned}
$$




$$
\begin{aligned}
& +2 \Re\left\{\mathbf{a}^{(1)}\left(\theta_{k}\right)^{H} \boldsymbol{\Omega}_{o} \mathbf{e}_{1} \mathbf{e}_{1}^{H} \mathbf{U}_{o} \mathbf{U}_{o}^{H} \mathbf{N V} \boldsymbol{\Sigma}_{s}^{-1} \mathbf{U}_{s}^{H} \mathbf{a}\left(\theta_{k}\right)\right. \\
& \left.\mathbf{a}^{(1)}\left(\theta_{k}\right)^{H} \mathbf{U}_{s} \Sigma_{s}^{-1} \mathbf{V}_{s}^{H} \mathbf{N}^{H} \mathbf{U}_{o} \mathbf{U}_{o}^{H} \mathbf{e}_{1} \mathbf{e}_{1}^{H} \boldsymbol{\Omega}_{o} \mathbf{a}^{(1)}\left(\theta_{k}\right)\right\} \\
& +2 \Re\left\{\mathbf{a}^{(1)}\left(\theta_{k}\right)^{H} \boldsymbol{\Omega}_{o} \mathbf{e}_{1} \mathbf{e}_{1}^{H} \mathbf{U}_{o} \mathbf{U}_{o}^{H} \mathrm{NV}_{s} \boldsymbol{\Sigma}_{s}^{-1} \mathbf{U}_{s}^{H} \mathbf{a}\left(\theta_{k}\right)\right. \\
& \left.\left.\mathbf{a}^{(1)}\left(\theta_{k}\right)^{H} \boldsymbol{\Omega}_{o} \mathbf{e}_{1} \mathbf{e}_{1}^{H} \mathbf{U}_{s} \boldsymbol{\Sigma}_{s}^{-1} \mathbf{V}_{s}^{H} \mathbf{N}^{H} \mathbf{U}_{o} \mathbf{U}_{o}^{H} \mathbf{a}^{(1)}\left(\theta_{k}\right)\right\}\right\} \\
& =\frac{2 \sigma_{n}^{2}}{\left\|\mathbf{e}_{1}^{H} \boldsymbol{\Omega}_{o}\right\|^{8}}\left\{\Re \left\{\mathbf{a}^{(1)}\left(\theta_{k}\right)^{H} \boldsymbol{\Omega}_{o} \mathbf{e}_{1} \mathbf{e}_{1}^{H} \mathbf{U}_{o} \mathbf{U}_{o}^{H} \mathbf{U}_{o} \mathbf{U}_{o}^{H} \mathbf{e}_{1} \mathbf{e}_{1}^{H} \boldsymbol{\Omega}_{o} \mathbf{a}^{(2)}\left(\theta_{k}\right)\right.\right. \\
& \left.\operatorname{Tr}\left[\mathbf{a}^{H}\left(\theta_{k}\right) \mathbf{U}_{s} \boldsymbol{\Sigma}_{s}^{-1} \mathbf{V}_{s}^{H} \mathbf{V}_{s} \boldsymbol{\Sigma}_{s}^{-1} \mathbf{U}_{s}^{H} \mathbf{a}\left(\theta_{k}\right)\right]\right\} \\
& +2 \Re\left\{\mathbf{a}^{(1)}\left(\theta_{k}\right)^{H} \boldsymbol{\Omega}_{o} \mathbf{e}_{1} \mathbf{e}_{1}^{H} \mathbf{U}_{o} \mathbf{U}_{o}^{H} \mathbf{U}_{o} \mathbf{U}_{o}^{H} \mathbf{e}_{1} \mathbf{e}_{1}^{H} \boldsymbol{\Omega}_{o} \mathbf{a}^{(1)}\left(\theta_{k}\right)\right. \\
& \left.\operatorname{Tr}\left[\mathbf{a}^{(1)}\left(\theta_{k}\right)^{H} \mathbf{U}_{s} \Sigma_{s}^{-1} \mathbf{V}_{s}^{H} \mathbf{V}_{s} \Sigma_{s}^{-1} \mathbf{U}_{s}^{H} \mathbf{a}\left(\theta_{k}\right)\right]\right\} \\
& +2 \Re\left\{\mathbf{a}^{(1)}\left(\theta_{k}\right)^{H} \boldsymbol{\Omega}_{o} \mathbf{e}_{1} \mathbf{e}_{1}^{H} \mathrm{U}_{o} \mathbf{U}_{o}^{H} \mathrm{U}_{o} \mathbf{U}_{o}^{H} \mathbf{a}^{(1)}\left(\theta_{k}\right)\right. \\
& \left.\left.\operatorname{Tr}\left[\mathbf{a}^{(1)}\left(\theta_{k}\right)^{H} \boldsymbol{\Omega}_{o} \mathbf{e}_{1} \mathbf{e}_{1}^{H} \mathbf{U}_{s} \boldsymbol{\Sigma}_{s}^{-1} \mathbf{V}_{s}^{H} \mathbf{V}_{s} \boldsymbol{\Sigma}_{s}^{-1} \mathbf{U}_{s}^{H} \mathbf{a}\left(\theta_{k}\right)\right]\right\}\right\} \\
& =\frac{2 \sigma_{n}^{2}}{\left\|\mathbf{e}_{1}^{H} \boldsymbol{\Omega}_{o}\right\|^{8}}\left\{\left\|\mathbf{e}_{1}^{H} \boldsymbol{\Omega}_{o}\right\|^{2} \Re\left\{\mathbf{a}^{(1)}\left(\theta_{k}\right)^{H} \boldsymbol{\Omega}_{o} \mathbf{e}_{1} \mathbf{e}_{1}^{H} \boldsymbol{\Omega}_{o} \mathbf{a}^{(2)}\left(\theta_{k}\right) \operatorname{Tr}\left[\mathbf{a}^{H}\left(\theta_{k}\right) \mathbf{U}_{s} \boldsymbol{\Sigma}_{s}^{-2} \mathbf{U}_{s}^{H} \mathbf{a}\left(\theta_{k}\right)\right]\right\}\right. \\
& +2\left|\mathbf{a}^{(1)}\left(\theta_{k}\right)^{H} \boldsymbol{\Omega}_{o} \mathbf{e}_{1}\left\|^{2}\right\| \mathbf{e}_{1}^{H} \boldsymbol{\Omega}_{o}\right|^{2} \Re\left\{\mathbf{a}^{(1)}\left(\theta_{k}\right)^{H} \mathbf{U}_{s} \boldsymbol{\Sigma}_{s}^{-2} \mathbf{U}_{s}^{H} \mathbf{a}\left(\theta_{k}\right)\right\} \\
& \left.+2\left|\mathbf{a}^{(1)}\left(\theta_{k}\right)^{H} \boldsymbol{\Omega}_{o} \mathbf{e}_{1}\right|^{2} \Re\left\{\mathbf{a}^{(1)}\left(\theta_{k}\right)^{H} \boldsymbol{\Omega}_{o} \mathbf{e}_{1} \mathbf{e}_{1}^{H} \mathbf{U}_{s} \boldsymbol{\Sigma}_{s}^{-2} \mathbf{U}_{s}^{H} \mathbf{a}\left(\theta_{k}\right)\right\}\right\} \text {. }
\end{aligned}
$$

Use (137) to obtain (92). 
APPENDIX C

BIAS DERIVATION FOR POLYNOMIAL ROOTING ALGORITHMS 
In this section, we derive the formulas (107)-(111) for Root MUSIC and (113)(111) for Root Min-Norm. Like extrema searching algorithms, the derivation here can be applicable to other polynomial root algorithms with slight difference in perturbed weighting matrix.

\section{C.1 ROOT MUSIC}

For Root MUSIC,

$$
P\left(r_{k}, \tilde{\mathbf{U}}_{o}\right)=\mathbf{a}\left(r_{k}^{-1}\right)^{T} \tilde{\Omega}_{o} \mathbf{a}\left(r_{k}\right)
$$

By definition (100),

$$
\begin{aligned}
N+\Delta N & \stackrel{\text { def }}{=} \frac{\partial P\left(r_{k}, \tilde{\mathbf{U}}_{o}\right)}{\partial z} \\
& =-r_{k}^{-2} \mathbf{a}^{(1)}\left(r_{k}^{-1}\right)^{T} \tilde{\mathbf{\Omega}}_{o} \mathbf{a}\left(r_{k}\right)+\mathbf{a}\left(r_{k}^{-1}\right)^{T} \tilde{\Omega}_{o} \mathbf{a}^{(1)}\left(r_{k}\right) \\
& =-2 j r_{k}^{-1} \Im\left\{r_{k}^{-1} \mathbf{a}^{(1)}\left(r_{k}^{-1}\right)^{T} \tilde{\Omega}_{o} \mathbf{a}\left(r_{k}\right)\right\}
\end{aligned}
$$

Keep the zeroth order term of (162) to obtain N,

$$
N=-2 j r_{k}^{-1} \Im\left\{r_{k}^{-1} \mathbf{a}^{(1)}\left(r_{k}^{-1}\right)^{T} \Omega_{o} \mathbf{a}\left(r_{k}\right)\right\}
$$

Use (8) to obtain (107). Clearly $\Delta N_{1}$ is the first-order term of $\Delta N$,

$$
\Delta N_{1}=-2 j r_{k}^{-1} \Im\left\{r_{k}^{-1} \mathbf{a}^{(1)}\left(r_{k}^{-1}\right)^{T} \Delta \Omega_{o 1} \mathbf{a}\left(r_{k}\right)\right\}
$$

Substitute $\Delta \boldsymbol{\Omega}_{o 1}$ into (163) and use (8) to obtain

$$
\begin{aligned}
\Delta N_{1} & =-2 j r_{k}^{-1} \Im\left\{r_{k}^{-1} \mathbf{a}^{(1)}\left(r_{k}^{-1}\right)^{T}\left[\Delta \mathbf{U}_{o 1} \mathbf{U}_{o}^{H}+\mathbf{U}_{o} \Delta \mathbf{U}_{o 1}^{H}\right] \mathbf{a}\left(r_{k}\right)\right\} \\
& =-2 j r_{k}^{-1} \Im\left\{r_{k}^{-1} \mathbf{a}^{(1)}\left(r_{k}^{-1}\right)^{T} \mathbf{U}_{o} \Delta \mathbf{U}_{o 1}^{H} \mathbf{a}\left(r_{k}\right)\right\} .
\end{aligned}
$$

$\Delta N_{2}$ is the second-order term of $\Delta N$,

$$
\Delta N_{2}=-2 j r_{k}^{-1} \Im\left\{r_{k}^{-1} \mathbf{a}^{(1)}\left(r_{k}^{-1}\right)^{T} \Delta \Omega_{o 2} \mathbf{a}\left(r_{k}\right)\right\}
$$


Substitute $\Delta \boldsymbol{\Omega}_{o 2}$ into (165) and use (8) to obtain

$$
\begin{aligned}
\Delta N_{2} & =-2 j r_{k}^{-1} \Im\left\{r_{k}^{-1} \mathbf{a}^{(1)}\left(r_{k}^{-1}\right)^{T}\left[\Delta \mathbf{U}_{o 2} \mathbf{U}_{o}^{H}+\mathbf{U}_{o} \Delta \mathbf{U}_{o 2}^{H}+\Delta \mathbf{U}_{o 1} \Delta \mathbf{U}_{o 1}^{H}\right] \mathbf{a}\left(r_{k}\right)\right\} \\
& =-2 j r_{k}^{-1} \Im\left\{r_{k}^{-1} \mathbf{a}^{(1)}\left(r_{k}^{-1}\right)^{T}\left[\mathbf{U}_{o} \Delta \mathbf{U}_{o 2}^{H}+\Delta \mathbf{U}_{o 1} \Delta \mathbf{U}_{o 1}^{H}\right] \mathbf{a}\left(r_{k}\right)\right\}
\end{aligned}
$$

By definition (100),

$$
\begin{aligned}
D+\Delta D \stackrel{\text { der }}{=} & \frac{\partial^{2} P\left(r_{k}, \tilde{\mathbf{U}}_{o}\right)}{\partial z^{2}} \\
= & 2 r_{k}^{-3} \mathbf{a}^{(1)}\left(r_{k}^{-1}\right)^{T} \tilde{\Omega}_{o} \mathbf{a}\left(r_{k}\right)+r_{k}^{-4} \mathbf{a}^{(2)}\left(r_{k}^{-1}\right)^{T} \tilde{\Omega}_{o} \mathbf{a}\left(r_{k}\right) \\
& -2 r_{k}^{-2} \mathbf{a}^{(1)}\left(r_{k}^{-1}\right)^{T} \tilde{\Omega}_{o} \mathbf{a}^{(1)}\left(r_{k}\right)+\mathbf{a}\left(r_{k}^{-1}\right)^{T} \tilde{\mathbf{\Omega}}_{o} \mathbf{a}^{(2)}\left(r_{k}\right) \\
= & 2 r_{k}^{-2} \Re\left\{r_{k}^{-2} \mathbf{a}^{(2)}\left(r_{k}^{-1}\right)^{T} \tilde{\Omega}_{o} \mathbf{a}\left(r_{k}\right)\right\} \\
& +2 r_{k}^{-3} \mathbf{a}^{(1)}\left(r_{k}^{-1}\right)^{T} \tilde{\Omega}_{o} \mathbf{a}\left(r_{k}\right)-2 r_{k}^{-2} \mathbf{a}^{(1)}\left(r_{k}^{-1}\right)^{T} \tilde{\Omega}_{o} \mathbf{a}^{(1)}\left(r_{k}\right) .
\end{aligned}
$$

Hence approximate (167) to the zeroth order term to obtain D,

$$
\begin{aligned}
D= & 2 r_{k}^{-2} \Re\left\{r_{k}^{-2} \mathbf{a}^{(2)}\left(r_{k}^{-1}\right)^{T} \Omega_{o} \mathbf{a}\left(r_{k}\right)\right\}+2 r_{k}^{-3} \mathbf{a}^{(1)}\left(r_{k}^{-1}\right)^{T} \Omega_{o} \mathbf{a}\left(r_{k}\right) \\
& -2 r_{k}^{-2} \mathbf{a}^{(1)}\left(r_{k}^{-1}\right)^{T} \Omega_{o} \mathbf{a}^{(1)}\left(r_{k}\right) .
\end{aligned}
$$

Use (8) to obtain (108). The first-order $\Delta D_{1}$ is obtained by keep (167) to the first-order and neglect the zeroth order term,

$$
\begin{aligned}
\Delta D_{1}= & 2 r_{k}^{-2} \Re\left\{r_{k}^{-2} \mathbf{a}^{(2)}\left(r_{k}^{-1}\right)^{T} \Delta \Omega_{o 1} \mathbf{a}\left(r_{k}\right)\right\}-2 r_{k}^{-2} \mathbf{a}^{(1)}\left(r_{k}^{-1}\right)^{T} \Delta \Omega_{o 1} \mathbf{a}^{(1)}\left(r_{k}\right) \\
& +2 r_{k}^{-3} \mathbf{a}^{(1)}\left(r_{k}^{-1}\right)^{T} \Delta \Omega_{o 1} \mathbf{a}\left(r_{k}\right) .
\end{aligned}
$$

Substitute $\Delta \boldsymbol{\Omega}_{o 1}$ into (168) and use (8) to obtain

$$
\begin{aligned}
& \Delta D_{1} \\
= & 2 r_{k}^{-3} \mathbf{a}^{(1)}\left(r_{k}^{-1}\right)^{T}\left[\Delta \mathbf{U}_{o 1} \mathbf{U}_{o}^{H}+\mathbf{U}_{o} \Delta \mathbf{U}_{o 1}^{H}\right] \mathbf{a}\left(r_{k}\right)-2 r_{k}^{-2} \mathbf{a}^{(1)}\left(r_{k}^{-1}\right)^{T}\left[\Delta \mathbf{U}_{o 1} \mathbf{U}_{o}^{H}+\mathbf{U}_{o} \Delta \mathbf{U}_{o 1}^{H}\right] \mathbf{a}^{(1)}\left(r_{k}\right) \\
& +2 r_{k}^{-2} \Re\left\{r_{k}^{-2} \mathbf{a}^{(2)}\left(r_{k}^{-1}\right)^{T}\left[\Delta \mathbf{U}_{o 1} \mathbf{U}_{o}^{H}+\mathbf{U}_{o} \Delta \mathbf{U}_{o 1}^{H}\right] \mathbf{a}\left(r_{k}\right)\right\}
\end{aligned}
$$




$$
\begin{aligned}
= & 2 r_{k}^{-2} \Re\left\{r_{k}^{-2} \mathbf{a}^{(2)}\left(r_{k}^{-1}\right)^{T} \mathbf{U}_{o} \Delta \mathbf{U}_{o 1}^{H} \mathbf{a}\left(r_{k}\right)\right\}-4 r_{k}^{-2} \Re\left\{\mathbf{a}^{(1)}\left(r_{k}^{-1}\right)^{T} \Delta \mathbf{U}_{o 1} \mathbf{U}_{o}^{H} \mathbf{a}^{(1)}\left(r_{k}\right)\right\} \\
& +2 r_{k}^{-3} \mathbf{a}^{(1)}\left(r_{k}^{-1}\right)^{T} \mathbf{U}_{o} \Delta \mathbf{U}_{o 1}^{H} \mathbf{a}\left(r_{k}\right) .
\end{aligned}
$$

By definition (100),

$$
\begin{aligned}
& B=\frac{\partial^{3} P\left(r_{k}, \mathbf{U}_{o}\right)}{\partial z^{3}} \\
& =-6 r_{k}^{-4} \mathbf{a}^{(1)}\left(r_{k}^{-1}\right)^{T} \Omega_{o} \mathbf{a}\left(r_{k}\right)-2 r_{k}^{-5} \mathbf{a}^{(2)}\left(r_{k}^{-1}\right)^{T} \Omega_{o} \mathbf{a}\left(r_{k}\right) \\
& +2 r_{k}^{-3} \mathbf{a}^{(1)}\left(r_{k}^{-1}\right)^{T} \Omega_{o} \mathbf{a}^{(1)}\left(r_{k}\right)-4 r_{k}^{-5} \mathbf{a}^{(2)}\left(r_{k}^{-1}\right)^{T} \Omega_{o} \mathbf{a}\left(r_{k}\right) \\
& -r_{k}^{-6} \mathbf{a}^{(3)}\left(r_{k}^{-1}\right)^{T} \Omega_{o} \mathbf{a}\left(r_{k}\right)+r_{k}^{-4} \mathbf{a}^{(2)}\left(r_{k}^{-1}\right)^{T} \Omega_{o} \mathbf{a}^{(1)}\left(r_{k}\right) \\
& +4 r_{k}^{-3} \mathbf{a}^{(1)}\left(r_{k}^{-1}\right)^{T} \Omega_{o} \mathbf{a}^{(1)}\left(r_{k}\right)+2 r_{k}^{-4} \mathbf{a}^{(2)}\left(r_{k}^{-1}\right)^{T} \Omega_{o} \mathbf{a}^{(1)}\left(r_{k}\right) \\
& -2 r_{k}^{-2} \mathbf{a}^{(1)}\left(r_{k}^{-1}\right)^{T} \Omega_{o} \mathbf{a}^{(2)}\left(r_{k}\right)-r_{k}^{-2} \mathbf{a}^{(2)}\left(r_{k}^{-1}\right)^{T} \Omega_{o} \mathbf{a}^{(1)}\left(r_{k}\right) \\
& +\mathbf{a}\left(r_{k}^{-1}\right)^{T} \Omega_{o} \mathbf{a}^{(3)}\left(r_{k}\right) \text {. }
\end{aligned}
$$

Use (8) to obtain (109). Substitute (69) into (165) and take expectation on both sides of the equation. Notice $\mathbf{a}^{H}\left(\theta_{k}\right) \mathbf{U}_{o}=0$, we have

$$
E\left(\Delta N_{2}\right)=-2 j r_{k}^{-1} \Im\left\{E\left\{r_{k}^{-1} \mathbf{a}^{(1)}\left(r_{k}^{-1}\right)^{T} \Delta \mathbf{U}_{o 1} \Delta \mathbf{U}_{o 1}^{H} \mathbf{a}\left(r_{k}\right)\right\}\right\}
$$

Substitute (58) and (136) back into the equation,

$$
\begin{aligned}
E\left(\Delta N_{2}\right) & =-2 j r_{k}^{-1} \Im\left\{E\left\{r_{k}^{-1} \mathbf{a}^{(1)}\left(r_{k}^{-1}\right)^{T} \mathbf{U}_{s} \Sigma_{s}^{-1} \mathbf{V}_{s}^{H} \mathbf{N}^{H} \mathbf{U}_{o} \mathbf{U}_{o}^{H} \mathbf{N V}_{s} \Sigma_{s}^{-1} \mathbf{U}_{s}^{H} \mathbf{a}\left(r_{k}\right)\right\}\right\} \\
& =-2 \sigma_{n}^{2} j r_{k}^{-1} \Im\left\{r_{k}^{-1} \mathbf{a}^{(1)}\left(r_{k}^{-1}\right)^{T} \mathrm{U}_{s} \Sigma_{s}^{-2} \mathbf{U}_{s}^{H} \mathbf{a}\left(r_{k}\right) \operatorname{Tr}\left(\mathbf{U}_{o} \mathbf{U}_{o}^{H}\right)\right\} \\
& =-2 j(L-P) \sigma_{n}^{2} r_{k}^{-1} \Im\left\{r_{k}^{-1} \mathbf{a}^{(1)}\left(r_{k}^{-1}\right)^{T} \mathbf{U}_{s} \Sigma_{s}^{-2} \mathbf{U}_{s}^{H} \mathbf{a}\left(r_{k}\right)\right\}
\end{aligned}
$$

Plug (137) into (170) to obtain (110). To calculate $E\left(\Delta^{2} N_{1}\right)$ from (164), we need to use the circularity of the noise,

$$
E\left(\Delta^{2} N_{1}\right)=-2 r_{k}^{-2} E\left\{\mathbf{a}^{(1)}\left(r_{k}^{-1}\right)^{T} \mathbf{U}_{o} \Delta \mathbf{U}_{o 1}^{H} \mathbf{a}\left(r_{k}\right) \mathbf{a}^{T}\left(r_{k}^{-1}\right) \Delta \mathbf{U}_{o} \mathbf{U}_{o}^{H} \mathbf{a}^{(1)}\left(r_{k}\right)\right\}
$$


Substitute (58) and (136) back into the equation,

$$
\begin{aligned}
& E\left(\Delta^{2} N_{1}\right) \\
= & -2 r_{k}^{-2} E\left\{\mathbf{a}^{(1)}\left(r_{k}^{-1}\right)^{T} \mathbf{U}_{o} \mathbf{U}_{o}^{H} \mathbf{N} \mathbf{V}_{s} \Sigma_{s}^{-1} \mathbf{U}_{s}^{H} \mathbf{a}\left(r_{k}\right) \mathbf{a}^{T}\left(r_{k}^{-1}\right) \mathbf{U}_{s} \Sigma_{s}^{-1} \mathbf{V}_{s}^{H} \mathbf{N}^{H} \mathbf{U}_{o} \mathbf{U}_{o}^{H} \mathbf{a}^{(1)}\left(r_{k}\right)\right\} \\
= & -2 \sigma_{n}^{2} r_{k}^{-2} \mathbf{a}^{(1)}\left(r_{k}^{-1}\right)^{T} \Omega_{o} \mathbf{a}^{(1)}\left(r_{k}\right) \operatorname{Tr}\left[\mathbf{a}^{T}\left(r_{k}^{-1}\right) \mathbf{U}_{s} \Sigma_{s}^{-2} \mathbf{U}_{s}^{H} \mathbf{a}\left(r_{k}\right)\right] \\
= & -2 \sigma_{n}^{2} r_{k}^{-2}\left\|\mathbf{a}^{(1)}\left(r_{k}^{-1}\right)^{T} \Omega_{o}\right\|^{2} \mathbf{a}^{T}\left(r_{k}^{-1}\right) \mathbf{U}_{s} \Sigma_{s}^{-2} \mathbf{U}_{s}^{H} \mathbf{a}\left(r_{k}\right) .
\end{aligned}
$$

Use (137) to obtain (111). Now we will use circular property of the noise to derive $E\left(\Delta N_{1} \Delta D_{1}\right)$. Then substitute (58) and (136) into the equation,

$$
\begin{aligned}
& E\left(\Delta N_{1} \Delta D_{1}\right) \\
& =2 j r_{k}^{-3} \Im\left\{E\left\{r_{k}^{-1} \mathbf{a}^{T}\left(r_{k}^{-1}\right) \Delta \mathbf{U}_{o 1} \mathbf{U}_{o}^{H} \mathbf{a}^{(1)}\left(r_{k}\right) \mathbf{a}^{(2)}\left(r_{k}^{-1}\right)^{T} \mathbf{U}_{o} \Delta \mathbf{U}_{o 1}^{H} \mathbf{a}\left(r_{k}\right)\right\}\right\} \\
& +4 j r_{k}^{-3} \Im\left\{E\left\{r_{k}^{-1} \mathbf{a}^{(1)}\left(r_{k}^{-1}\right)^{T} \mathbf{U}_{o} \Delta \mathbf{U}_{o 1}^{H} \mathbf{a}\left(r_{k}\right) \mathbf{a}^{(1)}\left(r_{k}^{-1}\right)^{T} \Delta \mathbf{U}_{o 1} \mathbf{U}_{o}^{H} \mathbf{a}^{(1)}\left(r_{k}\right)\right\}\right\} \\
& +2 j r_{k}^{-3} E\left\{\mathbf{a}^{T}\left(r_{k}^{-1}\right) \Delta \mathbf{U}_{o 1} \mathbf{U}_{o}^{H} \mathbf{a}^{(1)}\left(r_{k}\right) \mathbf{a}^{(1)}\left(r_{k}^{-1}\right)^{T} \mathrm{U}_{o} \Delta \mathbf{U}_{o 1}^{H} \mathbf{a}\left(r_{k}\right)\right\} \\
& =2 j r_{k}^{-3} \Im\left\{E\left\{r_{k}^{-1} \mathbf{a}^{T}\left(r_{k}^{-1}\right) \mathbf{U}_{s} \Sigma_{s}^{-1} \mathbf{V}_{s}^{H} \mathbf{N}^{H} \mathbf{U}_{o} \mathbf{U}_{o}^{H} \mathbf{a}^{(1)}\left(r_{k}\right) \mathbf{a}^{(2)}\left(r_{k}^{-1}\right)^{T} \mathbf{U}_{o} \mathbf{U}_{o}^{H} \mathbf{N} \mathbf{V}_{s} \boldsymbol{\Sigma}_{s}^{-1} \mathbf{U}_{s}^{H} \mathbf{a}\left(r_{k}\right)\right\}\right\} \\
& +4 j r_{k}^{-3} \Im\left\{E\left\{r_{k}^{-1} \mathbf{a}^{(1)}\left(r_{k}^{-1}\right)^{T} \mathbf{U}_{o} \mathbf{U}_{o}^{H} \mathbf{N V} \Sigma_{s}^{-1} \mathbf{U}_{s}^{H} \mathbf{a}\left(r_{k}\right) \mathbf{a}^{(1)}\left(r_{k}^{-1}\right)^{T} \mathbf{U}_{s} \Sigma_{s}^{-1} \mathbf{V}_{s}^{H} \mathbf{N}^{H} \mathbf{U}_{o} \mathbf{U}_{o}^{H} \mathbf{a}^{(1)}\left(r_{k}\right)\right\}\right\} \\
& +2 j r_{k}^{-3} E\left\{\mathbf{a}^{T}\left(r_{k}^{-1}\right) \mathbf{U}_{s} \Sigma_{s}^{-1} \mathbf{V}_{s}^{H} \mathbf{N}^{H} \mathbf{U}_{o} \mathbf{U}_{o}^{H} \mathbf{a}^{(1)}\left(r_{k}\right) \mathbf{a}^{(1)}\left(r_{k}^{-1}\right)^{T} \mathbf{U}_{o} \mathbf{U}_{o}^{H} \mathbf{N} \mathbf{V}_{s} \Sigma_{s}^{-1} \mathbf{U}_{s}^{H} \mathbf{a}\left(r_{k}\right)\right\} \\
& =2 j \sigma_{n}^{2} r_{k}^{-3} \Im\left\{r_{k}^{-1} \mathbf{a}^{T}\left(r_{k}^{-1}\right) \mathbf{U}_{s} \Sigma_{s}^{-2} \mathbf{U}_{s}^{H} \mathbf{a}\left(r_{k}\right) \operatorname{Tr}\left[\mathbf{a}^{(2)}\left(r_{k}^{-1}\right)^{T} \boldsymbol{\Omega}_{o} \mathbf{a}^{(1)}\left(r_{k}\right)\right]\right\} \\
& +4 j \sigma_{n}^{2} r_{k}^{-3} \Im\left\{r_{k}^{-1} \mathbf{a}^{(1)}\left(r_{k}^{-1}\right)^{T} \mathbf{\Omega}_{o} \mathbf{a}^{(1)}\left(r_{k}\right) \operatorname{Tr}\left[\mathbf{a}^{(1)}\left(r_{k}^{-1}\right)^{T} \mathbf{U}_{s} \Sigma_{s}^{-2} \mathbf{U}_{s}^{H} \mathbf{a}\left(r_{k}\right)\right]\right\} \\
& +2 j \sigma_{n}^{2} r_{k}^{-3} \mathbf{a}^{T}\left(r_{k}^{-1}\right) \mathbf{U}_{s} \Sigma_{s}^{-2} \mathbf{U}_{s}^{H} \mathbf{a}\left(r_{k}\right) \operatorname{Tr}\left[\mathbf{a}^{(1)}\left(r_{k}^{-1}\right)^{T} \Omega_{o} \mathbf{a}^{(1)}\left(r_{k}\right)\right] \\
& =2 j \sigma_{n}^{2} r_{k}^{-3} \Im\left\{r_{k}^{-1} \mathbf{a}^{T}\left(r_{k}^{-1}\right) \mathbf{U}_{s} \Sigma_{s}^{-2} \mathbf{U}_{s}^{H} \mathbf{a}\left(r_{k}\right) \mathbf{a}^{(2)}\left(r_{k}^{-1}\right)^{T} \mathbf{\Omega}_{o} \mathbf{a}^{(1)}\left(r_{k}\right)\right\} \\
& +4 j \sigma_{n}^{2} r_{k}^{-3}\left\|\mathbf{a}^{(1)}\left(r_{k}^{-1}\right)^{T} \Omega_{o}\right\|^{2} \Im\left\{r_{k}^{-1} \mathbf{a}^{(1)}\left(r_{k}^{-1}\right)^{T} \mathbf{U}_{s} \Sigma_{s}^{-2} \mathbf{U}_{s}^{H} \mathbf{a}\left(r_{k}\right)\right\} \\
& +2 j \sigma_{n}^{2} r_{k}^{-3} \mathbf{a}^{T}\left(r_{k}^{-1}\right) \mathbf{U}_{s} \Sigma_{s}^{-2} \mathbf{U}_{s}^{H} \mathbf{a}\left(r_{k}\right) \mathbf{a}^{(1)}\left(r_{k}^{-1}\right)^{T} \Omega_{o} \mathbf{a}^{(1)}\left(r_{k}\right) \text {. }
\end{aligned}
$$

Substitute (137) into the equation to get (112).

\section{C.2 ROOT MIN-NORM}


For Root Min-Norm,

$$
P\left(r_{k}, \tilde{\mathbf{U}}_{o}\right)=\frac{1}{\left\|\mathbf{e}_{1}^{H} \tilde{\mathbf{\Omega}}_{o}\right\|^{4}} \mathbf{a}\left(r_{k}^{-1}\right)^{T} \tilde{\mathbf{\Omega}}_{o} \mathbf{e}_{1} \mathbf{e}_{1}^{H} \tilde{\mathbf{\Omega}}_{o} \mathbf{a}\left(r_{k}\right)
$$

By definition (100),

$$
\begin{aligned}
N+\Delta N & \stackrel{\text { def }}{=} \frac{\partial P\left(r_{k}, \tilde{\mathbf{U}}_{o}\right)}{\partial z} \\
& =\frac{1}{\left\|\mathbf{e}_{1}^{H} \tilde{\Omega}_{o}\right\|^{4}}\left\{-r_{k}^{-2} \mathbf{a}^{(1)}\left(r_{k}^{-1}\right)^{T} \tilde{\Omega}_{o} \mathbf{e}_{1} \mathbf{e}_{1}^{H} \tilde{\Omega}_{o} \mathbf{a}\left(r_{k}\right)+\mathbf{a}\left(r_{k}^{-1}\right)^{T} \tilde{\Omega}_{o} \mathbf{e}_{1} \mathbf{e}_{1}^{H} \tilde{\mathbf{\Omega}}_{o} \mathbf{a}^{(1)}\left(r_{k}\right)\right\} \\
& =-\frac{2 j r_{k}^{-1}}{\left\|\mathbf{e}_{1}^{H} \tilde{\mathbf{\Omega}_{o}}\right\|^{4}} \Im\left\{r_{k}^{-1} \mathbf{a}^{(1)}\left(r_{k}^{-1}\right)^{T} \tilde{\Omega}_{o} \mathbf{e}_{1} \mathbf{e}_{1}^{H} \tilde{\mathbf{\Omega}}_{o} \mathbf{a}\left(r_{k}\right)\right\}
\end{aligned}
$$

$N$ is the zeroth order term of (171),

$$
N=-\frac{2 j r_{k}^{-1}}{\left\|\mathbf{e}_{1}^{H} \boldsymbol{\Omega}_{o}\right\|^{4}} \Im\left\{r_{k}^{-1} \mathbf{a}^{(1)}\left(r_{k}^{-1}\right)^{T} \boldsymbol{\Omega}_{o} \mathbf{e}_{1} \mathbf{e}_{1}^{H} \boldsymbol{\Omega}_{o} \mathbf{a}\left(r_{k}\right)\right\}
$$

Use (8) to obtain (113). $\Delta N_{1}$ is the first-order term of (171) excluding the zeroth order term,

$$
\Delta N_{1}=-\frac{2 j r_{k}^{-1}}{\left\|\mathbf{e}_{1}^{H} \boldsymbol{\Omega}_{o}\right\|^{4}} \Im\left\{r_{k}^{-1} \mathbf{a}^{(1)}\left(r_{k}^{-1}\right)^{T}\left[\boldsymbol{\Omega}_{o} \mathbf{e}_{1} \mathbf{e}_{1}^{H} \Delta \boldsymbol{\Omega}_{o 1}+\Delta \boldsymbol{\Omega}_{o 1} \mathbf{e}_{1} \mathbf{e}_{1}^{H} \boldsymbol{\Omega}_{o}\right] \mathbf{a}\left(r_{k}\right)\right\}
$$

Substitute $\Delta \boldsymbol{\Omega}_{o 1}$ into (172) and use (8) to obtain

$$
\begin{aligned}
\Delta N_{1} & =-\frac{2 j r_{k}^{-1}}{\left\|\mathbf{e}_{1}^{H} \Omega_{o}\right\|^{4}} \Im\left\{r_{k}^{-1} \mathbf{a}^{(1)}\left(r_{k}^{-1}\right)^{T} \Omega_{o} \mathbf{e}_{1} \mathbf{e}_{1}^{H} \Delta \boldsymbol{\Omega}_{o 1} \mathbf{a}\left(r_{k}\right)\right\} \\
& =-\frac{2 j r_{k}^{-1}}{\left\|\mathbf{e}_{1}^{H} \Omega_{o}\right\|^{4}} \Im\left\{r_{k}^{-1} \mathbf{a}^{(1)}\left(r_{k}^{-1}\right)^{T} \Omega_{o} \mathbf{e}_{1} \mathbf{e}_{1}^{H}\left[\Delta \mathbf{U}_{o 1} \mathbf{U}_{o}^{H}+\mathbf{U}_{o} \Delta \mathbf{U}_{o 1}^{H}\right] \mathbf{a}\left(r_{k}\right)\right\} \\
& =-\frac{2 j r_{k}^{-1}}{\left\|\mathbf{e}_{1}^{H} \boldsymbol{\Omega}_{o}\right\|^{4}} \Im\left\{r_{k}^{-1} \mathbf{a}^{(1)}\left(r_{k}^{-1}\right)^{T} \Omega_{o} \mathbf{e}_{1} \mathbf{e}_{1}^{H} \mathbf{U}_{o} \Delta \mathbf{U}_{o 1}^{H} \mathbf{a}\left(r_{k}\right)\right\}
\end{aligned}
$$

$\Delta N_{2}$ can be obtained by keeping (171) to the second-order and throwing away the zeroth order term,

$\Delta N_{2}=-\frac{2 j r_{k}^{-1}}{\left\|\mathbf{e}_{1}^{H} \boldsymbol{\Omega}_{o}\right\|^{4}} \Im\left\{r_{k}^{-1} \mathbf{a}^{(1)}\left(r_{k}^{-1}\right)^{T}\left[\boldsymbol{\Omega}_{o} \mathbf{e}_{1} \mathbf{e}_{1}^{H} \Delta \boldsymbol{\Omega}_{o 2}+\Delta \boldsymbol{\Omega}_{o 2} \mathbf{e}_{1} \mathbf{e}_{1}^{H} \boldsymbol{\Omega}_{o}+\Delta \boldsymbol{\Omega}_{o 1} \mathbf{e}_{1} \mathbf{e}_{1}^{H} \Delta \boldsymbol{\Omega}_{o 1}\right] \mathbf{a}\left(r_{k}\right)\right\}$ 
Substitute $\Delta \boldsymbol{\Omega}_{o 2}$ and $\Delta \boldsymbol{\Omega}_{o 1}$ into (174) and use (8) to obtain

$$
\begin{aligned}
\Delta N_{2}= & -\frac{2 j r_{k}^{-1}}{\left\|\mathbf{e}_{1}^{H} \Omega_{o}\right\|^{4}} \Im\left\{r_{k}^{-1} \mathbf{a}^{(1)}\left(r_{k}^{-1}\right)^{T}\left[\Omega_{o} \mathbf{e}_{1} \mathbf{e}_{1}^{H} \Delta \boldsymbol{\Omega}_{o 2}+\Delta \boldsymbol{\Omega}_{o 1} \mathbf{e}_{1} \mathbf{e}_{1}^{H} \Delta \boldsymbol{\Omega}_{o 1}\right] \mathbf{a}\left(r_{k}\right)\right\} \\
= & -\frac{2 j r_{k}^{-1}}{\left\|\mathbf{e}_{1}^{H} \boldsymbol{\Omega}_{o}\right\|^{4}} \Im\left\{r_{k}^{-1} \mathbf{a}^{(1)}\left(r_{k}^{-1}\right)^{T} \boldsymbol{\Omega}_{o} \mathbf{e}_{1} \mathbf{e}_{1}^{H}\left[\Delta \mathbf{U}_{o 2} \mathbf{U}_{o}^{H}+\mathbf{U}_{o} \Delta \mathbf{U}_{o 2}^{H}+\Delta \mathbf{U}_{o 1} \Delta \mathbf{U}_{o 1}^{H}\right] \mathbf{a}\left(r_{k}\right)\right. \\
& \left.+r_{k}^{-1} \mathbf{a}^{(1)}\left(r_{k}^{-1}\right)^{T}\left[\Delta \mathbf{U}_{o 1} \mathbf{U}_{o}^{H}+\mathbf{U}_{o} \Delta \mathbf{U}_{o 1}^{H}\right] \mathbf{e}_{1} \mathbf{e}_{1}^{H}\left[\Delta \mathbf{U}_{o 1} \mathbf{U}_{o}^{H}+\mathbf{U}_{o} \Delta \mathbf{U}_{o 1}^{H}\right] \mathbf{a}\left(r_{k}\right)\right\} \\
= & -\frac{2 j r_{k}^{-1}}{\left\|\mathbf{e}_{1}^{H} \boldsymbol{\Omega}_{o}\right\|^{4}} \Im\left\{r_{k}^{-1} \mathbf{a}^{(1)}\left(r_{k}^{-1}\right)^{T} \Omega_{o} \mathbf{e}_{1} \mathbf{e}_{1}^{H}\left[\mathbf{U}_{o} \Delta \mathbf{U}_{o 2}^{H}+\Delta \mathbf{U}_{o 1} \Delta \mathbf{U}_{o 1}^{H}\right] \mathbf{a}\left(r_{k}\right)\right. \\
& \left.+r_{k}^{-1} \mathbf{a}^{(1)}\left(r_{k}^{-1}\right)^{T}\left[\Delta \mathbf{U}_{o 1} \mathbf{U}_{o}^{H}+\mathbf{U}_{o} \Delta \mathbf{U}_{o 1}^{H}\right] \mathbf{e}_{1} \mathbf{e}_{1}^{H} \mathbf{U}_{o} \Delta \mathbf{U}_{o 1}^{H} \mathbf{a}\left(r_{k}\right)\right\} .
\end{aligned}
$$

By definition (100),

$$
\begin{aligned}
D+\Delta D \stackrel{\text { def }}{=} & \frac{\partial^{2} P\left(r_{k}, \tilde{\mathbf{U}}_{o}\right)}{\partial z^{2}} \\
= & \frac{1}{\left\|\mathbf{e}_{1}^{H} \tilde{\mathbf{\Omega}}_{o}\right\|^{4}}\left\{2 r_{k}^{-3} \mathbf{a}^{(1)}\left(r_{k}^{-1}\right)^{T} \tilde{\Omega}_{o} \mathbf{e}_{1} \mathbf{e}_{1}^{H} \tilde{\Omega}_{o} \mathbf{a}\left(r_{k}\right)+r_{k}^{-4} \mathbf{a}^{(2)}\left(r_{k}^{-1}\right)^{T} \tilde{\Omega}_{o} \mathbf{e}_{1} \mathbf{e}_{1}^{H} \tilde{\Omega}_{o} \mathbf{a}\left(r_{k}\right)\right. \\
& \left.-2 r_{k}^{-2} \mathbf{a}^{(1)}\left(r_{k}^{-1}\right)^{T} \tilde{\Omega}_{o} \mathbf{e}_{1} \mathbf{e}_{1}^{H} \tilde{\Omega}_{o} \mathbf{a}^{(1)}\left(r_{k}\right)+\mathbf{a}\left(r_{k}^{-1}\right)^{T} \tilde{\Omega}_{o} \mathbf{a}^{(2)}\left(r_{k}\right)\right\} \\
= & \frac{1}{\left\|\mathbf{e}_{1}^{H} \tilde{\mathbf{\Omega}}_{o}\right\|^{4}}\left\{2 r_{k}^{-2} \Re\left\{r_{k}^{-2} \mathbf{a}^{(2)}\left(r_{k}^{-1}\right)^{T} \tilde{\Omega}_{o} \mathbf{e}_{1} \mathbf{e}_{1}^{H} \tilde{\Omega}_{o} \mathbf{a}\left(r_{k}\right)\right\}\right. \\
& \left.+2 r_{k}^{-3} \mathbf{a}^{(1)}\left(r_{k}^{-1}\right)^{T} \tilde{\Omega}_{o} \mathbf{e}_{1} \mathbf{e}_{1}^{H} \tilde{\Omega}_{o} \mathbf{a}\left(r_{k}\right)-2 r_{k}^{-2} \mathbf{a}^{(1)}\left(r_{k}^{-1}\right)^{T} \tilde{\Omega}_{o} \mathbf{e}_{1} \mathbf{e}_{1}^{H} \tilde{\Omega}_{o} \mathbf{a}^{(1)}\left(r_{k}\right)\right\} .
\end{aligned}
$$

$D$ is the zeroth order term of the (176),

$$
\begin{aligned}
D= & \frac{1}{\left\|\mathbf{e}_{1}^{H} \boldsymbol{\Omega}_{o}\right\|^{4}}\left\{2 r_{k}^{-2} \Re\left\{r_{k}^{-2} \mathbf{a}^{(2)}\left(r_{k}^{-1}\right)^{T} \boldsymbol{\Omega}_{o} \mathbf{e}_{1} \mathbf{e}_{1}^{H} \boldsymbol{\Omega}_{o} \mathbf{a}\left(r_{k}\right)\right\}\right. \\
& \left.+2 r_{k}^{-3} \mathbf{a}^{(1)}\left(r_{k}^{-1}\right)^{T} \boldsymbol{\Omega}_{o} \mathbf{e}_{1} \mathbf{e}_{1}^{H} \boldsymbol{\Omega}_{o} \mathbf{a}\left(r_{k}\right)-2 r_{k}^{-2} \mathbf{a}^{(1)}\left(r_{k}^{-1}\right)^{T} \boldsymbol{\Omega}_{o} \mathbf{e}_{1} \mathbf{e}_{1}^{H} \boldsymbol{\Omega}_{o} \mathbf{a}^{(1)}\left(r_{k}\right)\right\} .
\end{aligned}
$$

Use (8) to obtain (114). Expanding (176) to first-order and neglect the zeroth order term to obtain $\Delta D_{1}$,

$$
\begin{aligned}
\Delta D_{1}= & \frac{1}{\left\|\mathbf{e}_{1}^{H} \boldsymbol{\Omega}_{o}\right\|^{4}}\left\{2 r_{k}^{-2} \Re\left\{r_{k}^{-2} \mathbf{a}^{(2)}\left(r_{k}^{-1}\right)^{T}\left[\Delta \boldsymbol{\Omega}_{o 1} \mathbf{e}_{1} \mathbf{e}_{1}^{H} \boldsymbol{\Omega}_{o}+\boldsymbol{\Omega}_{o} \mathbf{e}_{1} \mathbf{e}_{1}^{H} \Delta \boldsymbol{\Omega}_{o 1}\right] \mathbf{a}\left(r_{k}\right)\right\}\right. \\
& +2 r_{k}^{-3} \mathbf{a}^{(1)}\left(r_{k}^{-1}\right)^{T}\left[\Delta \boldsymbol{\Omega}_{o 1} \mathbf{e}_{1} \mathbf{e}_{1}^{H} \boldsymbol{\Omega}_{o}+\boldsymbol{\Omega}_{o} \mathbf{e}_{1} \mathbf{e}_{1}^{H} \Delta \boldsymbol{\Omega}_{o 1}\right] \mathbf{a}\left(r_{k}\right) \\
& \left.-2 r_{k}^{-2} \mathbf{a}^{(1)}\left(r_{k}^{-1}\right)^{T}\left[\Delta \boldsymbol{\Omega}_{o 1} \mathbf{e}_{1} \mathbf{e}_{1}^{H} \boldsymbol{\Omega}_{o}+\boldsymbol{\Omega}_{o} \mathbf{e}_{1} \mathbf{e}_{1}^{H} \Delta \boldsymbol{\Omega}_{o 1}\right] \mathbf{a}^{(1)}\left(r_{k}\right)\right\}
\end{aligned}
$$


Substitute $\Delta \boldsymbol{\Omega}_{o 1}$ into (177) and use (8) to obtain

$$
\begin{aligned}
& \Delta D_{1} \\
& =\frac{1}{\left\|\mathbf{e}_{1}^{H} \boldsymbol{\Omega}_{o}\right\|^{4}}\left\{2 r_{k}^{-2} \Re\left\{r_{k}^{-2} \mathbf{a}^{(2)}\left(r_{k}^{-1}\right)^{T} \boldsymbol{\Omega}_{o} \mathbf{e}_{1} \mathbf{e}_{1}^{H} \Delta \boldsymbol{\Omega}_{o 1} \mathbf{a}\left(r_{k}\right)\right\}\right. \\
& \left.-4 r_{k}^{-2} \Re\left\{\mathbf{a}^{(1)}\left(r_{k}^{-1}\right)^{T} \Delta \boldsymbol{\Omega}_{o 1} \mathbf{e}_{1} \mathbf{e}_{1}^{H} \boldsymbol{\Omega}_{o} \mathbf{a}^{(1)}\left(r_{k}\right)\right\}+2 r_{k}^{-3} \mathbf{a}^{(1)}\left(r_{k}^{-1}\right)^{T} \boldsymbol{\Omega}_{o} \mathbf{e}_{1} \mathbf{e}_{1}^{H} \Delta \boldsymbol{\Omega}_{o 1} \mathbf{a}\left(r_{k}\right)\right\} \\
& =\frac{1}{\left\|\mathbf{e}_{1}^{H} \boldsymbol{\Omega}_{o}\right\|^{4}}\left\{2 r_{k}^{-2} \Re\left\{r_{k}^{-2} \mathbf{a}^{(2)}\left(r_{k}^{-1}\right)^{T} \Omega_{o} \mathbf{e}_{1} \mathbf{e}_{1}^{H}\left[\Delta \mathbf{U}_{o 1} \mathbf{U}_{o}^{H}+\mathbf{U}_{o} \Delta \mathbf{U}_{o 1}^{H}\right] \mathbf{a}\left(r_{k}\right)\right\}\right. \\
& -4 r_{k}^{-2} \Re\left\{\mathbf{a}^{(1)}\left(r_{k}^{-1}\right)^{T}\left[\Delta \mathbf{U}_{o 1} \mathbf{U}_{o}^{H}+\mathbf{U}_{o} \Delta \mathbf{U}_{o 1}^{H}\right] \mathbf{e}_{1} \mathbf{e}_{1}^{H} \Omega_{o} \mathbf{a}^{(1)}\left(r_{k}\right)\right\} \\
& \left.+2 r_{k}^{-3} \mathbf{a}^{(1)}\left(r_{k}^{-1}\right)^{T} \boldsymbol{\Omega}_{o} \mathbf{e}_{1} \mathbf{e}_{1}^{H}\left[\Delta \mathbf{U}_{o 1} \mathbf{U}_{o}^{H}+\mathbf{U}_{o} \Delta \mathbf{U}_{o 1}^{H}\right] \mathbf{a}\left(r_{k}\right)\right\} \\
& =\frac{1}{\left\|\mathbf{e}_{1}^{H} \Omega_{o}\right\|^{4}}\left\{2 r_{k}^{-2} \Re\left\{r_{k}^{-2} \mathbf{a}^{(2)}\left(r_{k}^{-1}\right)^{T} \Omega_{o} \mathbf{e}_{1} \mathbf{e}_{1}^{H} \mathbf{U}_{o} \Delta \mathbf{U}_{o 1}^{H} \mathbf{a}\left(r_{k}\right)\right\}\right. \\
& -4 r_{k}^{-2} \Re\left\{\mathbf{a}^{(1)}\left(r_{k}^{-1}\right)^{T}\left[\Delta \mathbf{U}_{o 1} \mathbf{U}_{o}^{H}+\mathbf{U}_{o} \Delta \mathbf{U}_{o 1}^{H}\right] \mathbf{e}_{1} \mathbf{e}_{1}^{H} \boldsymbol{\Omega}_{o} \mathbf{a}^{(1)}\left(r_{k}\right)\right\} \\
& \left.+2 r_{k}^{-3} \mathbf{a}^{(1)}\left(r_{k}^{-1}\right)^{T} \Omega_{o} \mathbf{e}_{1} \mathbf{e}_{1}^{H} \mathbf{U}_{o} \Delta \mathbf{U}_{o 1}^{H} \mathbf{a}\left(r_{k}\right)\right\} \text {. }
\end{aligned}
$$

$B$, by definition, is the zeroth term of $\frac{\partial^{3} P\left(r_{k}, \tilde{U}_{o}\right)}{\partial z^{3}}$, i.e.,

$$
\begin{aligned}
& B=\frac{\partial^{3} P\left(r_{k}, \mathbf{U}_{o}\right)}{\partial z^{3}} \\
& =\frac{1}{\left\|\mathbf{e}_{1}^{H} \boldsymbol{\Omega}_{o}\right\|^{4}}\left\{-6 r_{k}^{-4} \mathbf{a}^{(1)}\left(r_{k}^{-1}\right)^{T} \boldsymbol{\Omega}_{o} \mathbf{e}_{1} \mathbf{e}_{1}^{H} \boldsymbol{\Omega}_{o} \mathbf{a}\left(r_{k}\right)-2 r_{k}^{-5} \mathbf{a}^{(2)}\left(r_{k}^{-1}\right)^{T} \Omega_{o} \mathbf{e}_{1} \mathbf{e}_{1}^{H} \boldsymbol{\Omega}_{o} \mathbf{a}\left(r_{k}\right)\right. \\
& +2 r_{k}^{-3} \mathbf{a}^{(1)}\left(r_{k}^{-1}\right)^{T} \boldsymbol{\Omega}_{o} \mathbf{e}_{1} \mathbf{e}_{1}^{H} \boldsymbol{\Omega}_{o} \mathbf{a}^{(1)}\left(r_{k}\right)-4 r_{k}^{-5} \mathbf{a}^{(2)}\left(r_{k}^{-1}\right)^{T} \boldsymbol{\Omega}_{o} \mathbf{e}_{1} \mathbf{e}_{1}^{H} \boldsymbol{\Omega}_{o} \mathbf{a}\left(r_{k}\right) \\
& -r_{k}^{-6} \mathbf{a}^{(3)}\left(r_{k}^{-1}\right)^{T} \boldsymbol{\Omega}_{o} \mathbf{e}_{1} \mathbf{e}_{1}^{H} \boldsymbol{\Omega}_{o} \mathbf{a}\left(r_{k}\right)+r_{k}^{-4} \mathbf{a}^{(2)}\left(r_{k}^{-1}\right)^{T} \boldsymbol{\Omega}_{o} \mathbf{e}_{1} \mathbf{e}_{1}^{H} \boldsymbol{\Omega}_{o} \mathbf{a}^{(1)}\left(r_{k}\right) \\
& +4 r_{k}^{-3} \mathbf{a}^{(1)}\left(r_{k}^{-1}\right)^{T} \boldsymbol{\Omega}_{o} \mathbf{e}_{1} \mathbf{e}_{1}^{H} \boldsymbol{\Omega}_{o} \mathbf{a}^{(1)}\left(r_{k}\right)+2 r_{k}^{-4} \mathbf{a}^{(2)}\left(r_{k}^{-1}\right)^{T} \boldsymbol{\Omega}_{o} \mathbf{e}_{1} \mathbf{e}_{1}^{H} \boldsymbol{\Omega}_{o} \mathbf{a}^{(1)}\left(r_{k}\right) \\
& -2 r_{k}^{-2} \mathbf{a}^{(1)}\left(r_{k}^{-1}\right)^{T} \boldsymbol{\Omega}_{o} \mathbf{e}_{1} \mathbf{e}_{1}^{H} \boldsymbol{\Omega}_{o} \mathbf{a}^{(2)}\left(r_{k}\right)-r_{k}^{-2} \mathbf{a}^{(2)}\left(r_{k}^{-1}\right)^{T} \boldsymbol{\Omega}_{o} \mathbf{e}_{1} \mathbf{e}_{1}^{H} \boldsymbol{\Omega}_{o} \mathbf{a}^{(1)}\left(r_{k}\right) \\
& \left.+\mathbf{a}\left(r_{k}^{-1}\right)^{T} \boldsymbol{\Omega}_{o} \mathbf{e}_{1} \mathbf{e}_{1}^{H} \boldsymbol{\Omega}_{o} \mathbf{a}^{(3)}\left(r_{k}\right)\right\} .
\end{aligned}
$$

Use (8) to obtain (115). Substitute (69) into (175) and take expectation on both sides of 
the equation. Using $\mathbf{a}^{H}\left(\theta_{k}\right) \mathbf{U}_{o}=0$ and the circular property of the noise, we have

$$
\begin{aligned}
E\left(\Delta N_{2}\right)= & -\frac{2 j r_{k}^{-1}}{\left\|\mathbf{e}_{1}^{H} \boldsymbol{\Omega}_{o}\right\|^{4}} E\left\{\Im \left\{r_{k}^{-1} \mathbf{a}^{(1)}\left(r_{k}^{-1}\right)^{T} \mathbf{\Omega}_{o} \mathbf{e}_{1} \mathbf{e}_{1}^{H} \Delta \mathbf{U}_{o 1} \Delta \mathbf{U}_{o 1}^{H} \mathbf{a}\left(r_{k}\right)\right.\right. \\
& \left.\left.+r_{k}^{-1} \mathbf{a}^{(1)}\left(r_{k}^{-1}\right)^{T} \Delta \mathbf{U}_{o 1} \mathbf{U}_{o}^{H} \mathbf{e}_{1} \mathbf{e}_{1}^{H} \mathrm{U}_{o} \Delta \mathbf{U}_{o 1}^{H} \mathbf{a}\left(r_{k}\right)\right\}\right\}
\end{aligned}
$$

Substitute (58) and (136) back into the equation,

$$
\begin{aligned}
& E\left(\Delta N_{2}\right) \\
& =-\frac{2 j r_{k}^{-1}}{\left\|\mathbf{e}_{1}^{H} \Omega_{o}\right\|^{4}} \Im\left\{E\left[r_{k}^{-1} \mathbf{a}^{(1)}\left(r_{k}^{-1}\right)^{T} \Omega_{o} \mathbf{e}_{1} \mathbf{e}_{1}^{H} \mathbf{U}_{s} \Sigma_{s}^{-1} \mathbf{V}_{s}^{H} \mathbf{N}^{H} \mathbf{U}_{o} \mathbf{U}_{o}^{H} \mathbf{N V} \boldsymbol{\Sigma}_{s}^{-1} \mathrm{U}_{s}^{H} \mathbf{a}\left(r_{k}\right)\right]\right\} \\
& \left.+E\left[r_{k}^{-1} \mathbf{a}^{(1)}\left(r_{k}^{-1}\right)^{T} \mathbf{U}_{s} \Sigma_{s}^{-1} \mathbf{V}_{s}^{H} \mathbf{N}^{H} \mathbf{U}_{o} \mathbf{U}_{o}^{H} \mathbf{e}_{1} \mathbf{e}_{1}^{H} \mathbf{U}_{o} \mathbf{U}_{o}^{H} \mathbf{N V} \Sigma_{s}^{-1} \mathbf{U}_{s}^{H} \mathbf{a}\left(r_{k}\right)\right]\right\} \\
& =-\frac{2 j \sigma_{n}^{2} r_{k}^{-1}}{\left\|\mathbf{e}_{1}^{H} \Omega_{o}\right\|^{4}} \Im\left\{r_{k}^{-1} \mathbf{a}^{(1)}\left(r_{k}^{-1}\right)^{T} \Omega_{o} \mathbf{e}_{1} \mathbf{e}_{1}^{H} \mathbf{U}_{s} \Sigma_{s}^{-2} \mathbf{U}_{s}^{H} \mathbf{a}\left(r_{k}\right) \operatorname{Tr}\left[\mathbf{U}_{o} \mathbf{U}_{o}^{H}\right]\right. \\
& \left.+r_{k}^{-1} \mathbf{a}^{(1)}\left(r_{k}^{-1}\right)^{T} \mathbf{U}_{s} \Sigma_{s}^{-2} \mathbf{U}_{s}^{H} \mathbf{a}\left(r_{k}\right) \operatorname{Tr}\left[\mathbf{e}_{1}^{H} \Omega_{o} \mathbf{e}_{1}\right]\right\} \\
& =-\frac{2 j \sigma_{n}^{2} r_{k}^{-1}}{\left\|\mathbf{e}_{1}^{H} \Omega_{o}\right\|^{4}} \Im\left\{(L-P) r_{k}^{-1} \mathbf{a}^{(1)}\left(r_{k}^{-1}\right)^{T} \Omega_{o} \mathbf{e}_{1} \mathbf{e}_{1}^{H} \mathbf{U}_{s} \Sigma_{s}^{-2} \mathbf{U}_{s}^{H} \mathbf{a}\left(r_{k}\right)\right. \\
& \left.+\left\|\mathrm{e}_{1} \Omega_{o}\right\|^{2} r_{k}^{-1} \mathbf{a}^{(1)}\left(r_{k}^{-1}\right)^{T} \mathbf{U}_{s} \Sigma_{s}^{-2} \mathbf{U}_{s}^{H} \mathrm{a}\left(r_{k}\right)\right\} \text {. }
\end{aligned}
$$

Use (137) to obtain (116). For $E\left(\Delta^{2} N_{1}\right)$, using the circularity of the noise and substituting (58) and (136) into the equation, we have

$$
\begin{aligned}
& E\left(\Delta^{2} N_{1}\right) \\
= & \frac{-2 r_{k}^{-2}}{\left\|\mathbf{e}_{1}^{H} \boldsymbol{\Omega}_{o}\right\|^{8}} E\left[\mathbf{a}^{(1)}\left(r_{k}^{-1}\right)^{T} \Omega_{o} \mathbf{e}_{1} \mathbf{e}_{1}^{H} \mathbf{U}_{o} \Delta \mathbf{U}_{o 1}^{H} \mathbf{a}\left(r_{k}\right) \mathbf{a}^{T}\left(r_{k}^{-1}\right) \Delta \mathbf{U}_{o 1} \mathbf{U}_{o}^{H} \mathbf{e}_{1} \mathbf{e}_{1}^{H} \Omega_{o} \mathbf{a}^{(1)}\left(r_{k}\right)\right] \\
= & \frac{-2 \sigma_{n}^{2} r_{k}^{-2}}{\left\|\mathbf{e}_{1}^{H} \Omega_{o}\right\|^{8}} \mathbf{a}^{(1)}\left(r_{k}^{-1}\right)^{T} \Omega_{o} \mathbf{e}_{1} \mathbf{e}_{1}^{H} \Omega_{o} \mathbf{e}_{1} \mathbf{e}_{1}^{H} \Omega_{o} \mathbf{a}^{(1)}\left(r_{k}\right) \operatorname{Tr}\left[\mathbf{a}^{T}\left(r_{k}^{-1}\right) \mathbf{U}_{s} \Sigma_{s}^{-2} \mathbf{U}_{s}^{H} \mathbf{a}\left(r_{k}\right)\right] \\
= & \frac{-2 \sigma_{n}^{2} r_{k}^{-2}}{\left\|\mathbf{e}_{1}^{H} \Omega_{o}\right\|^{8}}\left\|\mathbf{a}^{(1)}\left(r_{k}^{-1}\right)^{T} \Omega_{o} \mathbf{e}_{1}\right\|^{2}\left\|\mathbf{e}_{1}^{H} \Omega_{o}\right\|^{2} \mathbf{a}^{T}\left(r_{k}^{-1}\right) \mathbf{U}_{s} \Sigma_{s}^{-2} U_{s}^{H} \mathbf{a}\left(r_{k}\right) .
\end{aligned}
$$

Use (137) to obtain (117). To calculate $E\left(\Delta N_{1} \Delta D_{1}\right)$, we utilize the circular property of the noise then substitute (58) and (136),

$$
E\left(\Delta N_{1} \Delta D_{1}\right)
$$




$$
\begin{aligned}
& =\frac{1}{\left\|\mathbf{e}_{1}^{H} \Omega_{o}\right\|^{8}}\left\{2 j r _ { k } ^ { - 3 } \Im \left\{E \left[r_{k}^{-1} \mathbf{a}^{T}\left(r_{k}^{-1}\right) \Delta \mathbf{U}_{o 1} \mathbf{U}_{o}^{H} \mathbf{e}_{1} \mathbf{e}_{1}^{H} \Omega_{o} \mathbf{a}^{(1)}\left(r_{k}\right)\right.\right.\right. \\
& \left.\left.\mathbf{a}^{(2)}\left(r_{k}^{-1}\right)^{T} \boldsymbol{\Omega}_{o} \mathbf{e}_{1} \mathbf{e}_{1}^{H} \mathbf{U}_{o} \Delta \mathbf{U}_{o 1}^{H} \mathbf{a}\left(r_{k}\right)\right]\right\} \\
& +4 j r_{k}^{-3} \Im\left\{E \left[r_{k}^{-1} \mathbf{a}^{(1)}\left(r_{k}^{-1}\right)^{T} \Omega_{o} \mathbf{e}_{1} \mathbf{e}_{1}^{H} \mathbf{U}_{o} \Delta \mathbf{U}_{o 1}^{H} \mathbf{a}\left(r_{k}\right)\right.\right. \\
& \left.\left.\mathbf{a}^{(1)}\left(r_{k}^{-1}\right)^{T} \Delta \mathbf{U}_{o 1} \mathbf{U}_{o}^{H} \mathbf{e}_{1} \mathbf{e}_{1}^{H} \boldsymbol{\Omega}_{o} \mathbf{a}^{(1)}\left(r_{k}\right)\right]\right\} \\
& +4 j r_{k}^{-3} \Im\left\{E \left[r_{k}^{-1} \mathbf{a}^{(1)}\left(r_{k}^{-1}\right)^{T} \boldsymbol{\Omega}_{o} \mathbf{e}_{1} \mathbf{e}_{1}^{H} \mathbf{U}_{o} \Delta \mathbf{U}_{o 1}^{H} \mathbf{a}\left(r_{k}\right)\right.\right. \\
& \left.\left.\mathbf{a}^{(1)}\left(r_{k}^{-1}\right)^{T} \Omega_{o} \mathbf{e}_{1} \mathbf{e}_{1}^{H} \Delta \mathbf{U}_{o 1} \mathbf{U}_{o}^{H} \mathbf{a}^{(1)}\left(r_{k}\right)\right]\right\} \\
& \left.+2 r_{k}^{-3} E\left[\mathbf{a}^{T}\left(r_{k}^{-1}\right) \Delta \mathbf{U}_{o 1} \mathbf{U}_{o}^{H} \mathbf{e}_{1} \mathbf{e}_{1}^{H} \boldsymbol{\Omega}_{o} \mathbf{a}^{(1)}\left(r_{k}\right) \mathbf{a}^{(1)}\left(r_{k}^{-1}\right)^{T} \boldsymbol{\Omega}_{o} \mathbf{e}_{1} \mathbf{e}_{1}^{H} \mathbf{U}_{o} \Delta \mathbf{U}_{o 1}^{H} \mathbf{a}\left(r_{k}\right)\right]\right\} \\
& =\frac{1}{\left\|\mathbf{e}_{1}^{H} \Omega_{o}\right\|^{8}}\left\{2 j \sigma _ { n } ^ { 2 } r _ { k } ^ { - 3 } \Im \left\{r_{k}^{-1} \mathbf{a}^{T}\left(r_{k}^{-1}\right) \mathbf{U}_{s} \boldsymbol{\Sigma}_{s}^{-2} \mathbf{U}_{s}^{H} \mathbf{a}\left(r_{k}\right)\right.\right. \\
& \left.\operatorname{Tr}\left[\mathbf{a}^{(2)}\left(r_{k}^{-1}\right)^{T} \mathbf{\Omega}_{o} \mathbf{e}_{1} \mathbf{e}_{1}^{H} \boldsymbol{\Omega}_{o} \mathbf{e}_{1} \mathbf{e}_{1}^{H} \boldsymbol{\Omega}_{o} \mathbf{a}^{(1)}\left(r_{k}\right)\right]\right\} \\
& +4 j \sigma_{n}^{2} r_{k}^{-3} \Im\left\{r_{k}^{-1} \mathbf{a}^{(1)}\left(r_{k}^{-1}\right)^{T} \boldsymbol{\Omega}_{o} \mathbf{e}_{1} \mathbf{e}_{1}^{H} \boldsymbol{\Omega}_{o} \mathbf{e}_{1} \mathbf{e}_{1}^{H} \boldsymbol{\Omega}_{o} \mathbf{a}^{(1)}\left(r_{k}\right)\right. \\
& \left.\operatorname{Tr}\left[\mathbf{a}^{(1)}\left(r_{k}^{-1}\right)^{T} \mathbf{U}_{s} \Sigma_{s}^{-2} \mathbf{U}_{s}^{H} \mathbf{a}\left(r_{k}\right)\right]\right\} \\
& +4 j \sigma_{n}^{2} r_{k}^{-3} \Im\left\{r_{k}^{-1} \mathbf{a}^{(1)}\left(r_{k}^{-1}\right)^{T} \Omega_{o} \mathbf{e}_{1} \mathbf{e}_{1}^{H} \mathbf{\Omega}_{o} \mathbf{a}^{(1)}\left(r_{k}\right)\right. \\
& \left.\operatorname{Tr}\left[\mathbf{a}^{(1)}\left(r_{k}^{-1}\right)^{T} \Omega_{o} \mathbf{e}_{1} \mathbf{e}_{1}^{H} \mathrm{U}_{s} \Sigma_{s}^{-2} \mathrm{U}_{s}^{H} \mathbf{a}\left(r_{k}\right)\right]\right\} \\
& \left.+2 \sigma_{n}^{2} r_{k}^{-3} \mathbf{a}^{T}\left(r_{k}^{-1}\right) \mathrm{U}_{s} \Sigma_{s}^{-2} \mathbf{U}_{s}^{H} \mathbf{a}\left(r_{k}\right) \operatorname{Tr}\left[\mathbf{a}^{(1)}\left(r_{k}^{-1}\right)^{T} \Omega_{o} \mathbf{e}_{1} \mathbf{e}_{1}^{H} \Omega_{o} \mathbf{e}_{1} \mathbf{e}_{1}^{H} \boldsymbol{\Omega}_{o} \mathbf{a}^{(1)}\left(r_{k}\right)\right]\right\}
\end{aligned}
$$

Use (137) to obtain (118). 
APPENDIX D

BIAS DERIVATION FOR MATRIX-SHIFTING ALGORITHMS 
In this section, the derivation of $(128)$ is presented.

\section{D.1 EIGENVALUE PERTURBATION}

From [24], the first-order approximation of eigenvalue perturbation is

$$
\Delta \lambda_{k 1}=\mathbf{y}_{k}^{H} \Delta \mathbf{F}_{1} \mathbf{x}_{k}
$$

where $\mathbf{y}_{k}^{H}$ is the left eigenvector and $\mathbf{x}_{k}$ is the right eigenvector of $\mathbf{F}$, i.e.,

$$
\mathbf{y}_{k}^{H}=\mathbf{e}_{k}^{H} \mathbf{L}^{-1} \text { and } \mathbf{x}_{k}=\mathbf{L} \mathbf{e}_{k}
$$

where $\mathbf{e}_{k}$ is a column vector with all zero elements except the $k$-th element is 1 . The second-order approximation is

$$
\Delta \lambda_{k 2}=\mathbf{y}_{k}^{H} \Delta \mathbf{F}_{2} \mathbf{x}_{k}+\mathbf{y}_{k}^{H} \Delta \mathbf{F}_{1} \Delta \mathbf{x}_{k 1}
$$

where

$$
\Delta \mathbf{x}_{k 1}=\left(\lambda_{k} \mathbf{I}-\mathbf{F}\right)^{-1}\left(\Delta \mathbf{F}_{1}-\mathbf{y}_{k}^{H} \Delta \mathbf{F}_{1} \mathbf{x}_{k} \mathbf{I}\right) \mathbf{x}_{k}
$$

Substitute (183) into (182), we obtain

$$
\Delta \lambda_{k 2}=\mathbf{y}_{k}^{H} \Delta \mathbf{F}_{2} \mathbf{x}_{k}+\mathbf{y}_{k}^{H} \Delta \mathbf{F}_{1}\left(\lambda_{k} \mathbf{I}-\mathbf{F}\right)^{-1}\left(\Delta \mathbf{F}_{1}-\mathbf{y}_{k}^{H} \Delta \mathbf{F}_{1} \mathbf{x}_{k} \mathbf{I}\right) \mathbf{x}_{k} .
$$

Take the expectation of $\Delta \lambda_{k 2}$, we have

$$
E\left(\Delta \lambda_{k 2}\right)=E\left(\mathbf{y}_{k}^{H} \Delta \mathbf{F}_{2} \mathbf{x}_{k}\right)+E\left[\mathbf{y}_{k}^{H} \Delta \mathbf{F}_{1}\left(\lambda_{k} \mathbf{I}-\mathbf{F}\right)^{-1}\left(\Delta \mathbf{F}_{1}-\mathbf{y}_{k}^{H} \Delta \mathbf{F}_{1} \mathbf{x}_{k} \mathbf{I}\right) \mathbf{x}_{k}\right]
$$

The second term is zero because of the circularity of noise as $\Delta F_{1}$ is linear to $\Delta U_{s 1}$ and thus linear to $\mathbf{N}$ [5]. The first term can be further simplified by substituting (127) into (185)

$$
\begin{aligned}
& E\left(\Delta \lambda_{k 2}\right)=\underbrace{\mathbf{y}_{k}^{H} E\left[\mathbf{U}_{s}^{\dagger \dagger}\left(\Delta \mathbf{U}_{s 2}^{\dagger}-\Delta \mathbf{U}_{s 2}^{!} \mathbf{F}\right)\right] \mathbf{x}_{k}}_{T 1} \underbrace{-\mathbf{y}_{k}^{H} E\left[\mathbf{U}_{s}^{\dagger}{ }^{\dagger} \Delta \mathbf{U}_{s 1}^{!} \mathbf{U}_{s}^{\dagger}\left(\Delta \mathbf{U}_{s 1}^{\dagger}-\Delta \mathbf{U}_{s 1}^{!} \mathbf{F}\right)\right] \mathbf{x}_{k}}_{T 2} \\
& \underbrace{+\mathbf{y}_{k}^{H} E\left[\left(\mathbf{U}_{s}^{\rfloor^{H}} \mathbf{U}_{s}^{\dagger}\right)^{-1} \Delta \mathbf{U}_{s 1}^{\downarrow^{H}}\left(\mathbf{I}-\mathbf{U}_{s}^{\lfloor} \mathbf{U}_{s}^{\dagger}\right)\left(\Delta \mathbf{U}_{s 1}^{\dagger}-\Delta \mathbf{U}_{s 1}^{\dagger} \mathbf{F}\right)\right] \mathbf{x}_{k}}_{T 3}
\end{aligned}
$$


The second term is zero because of circularity. To simplify the first and third-term, notice that from (15), (124) and (122) with $\mathrm{W}$ equal to I

$$
\mathbf{U}_{s}^{\downarrow}=\mathbf{A}^{\downarrow} \mathbf{L}^{-1} \text { and } \mathbf{U}_{s}^{\downarrow^{H}}=\left(\mathbf{L}^{-1}\right)^{H} \mathbf{A}^{\downarrow^{H}}
$$

so that

$$
\left(\mathbf{U}_{s}^{!^{H}} \mathbf{U}_{s}^{\downarrow}\right)^{-1}=\mathbf{L}\left(\mathbf{A}^{\downarrow^{H}} \mathbf{A}^{\downarrow}\right)^{-1} \mathbf{L}^{H}
$$

and

$$
\begin{aligned}
\mathbf{U}_{s}^{\downarrow^{\dagger}} & =\mathbf{L}\left(\mathbf{A}^{\downarrow^{H}} \mathbf{A}^{\downarrow}\right)^{-1} \mathbf{A}^{\downarrow^{H}}=\mathbf{L} \mathbf{A}^{\downarrow^{\dagger}} \\
\mathbf{U}_{s}^{\downarrow} \mathbf{U}_{s}^{\downarrow^{\dagger}} & =\mathbf{A}^{\downarrow}\left(\mathbf{A}^{\downarrow^{H}} \mathbf{A}^{\downarrow}\right)^{-1} \mathbf{A}^{\downarrow^{H}} \stackrel{\text { def }}{=} \mathbf{P}_{\mathbf{A} \downarrow}
\end{aligned}
$$

where $\mathbf{P}_{\mathbf{A} \downarrow}$ is the projection matrix of $\mathbf{A}^{\downarrow}$. Also notice that

$$
\mathbf{F L} \mathbf{e}_{k}=\mathbf{L D} \mathbf{e}_{k}=\mathbf{L} \lambda_{k} \mathbf{e}_{k}
$$

Now substitute (189), (191), (181), (70) and $\mathbf{U}_{\boldsymbol{s}}=\mathbf{A L}^{-1}$ as can be seen directly from (187) into the first term of (186), we obtain

$$
\begin{aligned}
T 1 & =-\frac{1}{2} \mathbf{e}_{k}^{H} \mathbf{A}^{\downarrow^{\dagger}}\left(\mathbf{I}^{\dagger}-\lambda_{k} \mathbf{I}^{\downarrow}\right) \mathbf{U}_{s} \Sigma_{s}^{-2} \operatorname{Tr}\left(\mathbf{U}_{o} \mathbf{U}_{o}^{H}\right) \sigma_{n}^{2} \mathbf{L} \mathbf{e}_{k} \\
& =-\frac{1}{2} \operatorname{Tr}\left(\mathbf{U}_{o} \mathbf{U}_{o}^{H}\right) \sigma_{n}^{2} \lambda_{k} \mathbf{e}_{k}^{H}\left(\mathbf{D}^{-1} \mathbf{A}^{\downarrow^{\dagger}} \mathbf{I}^{\dagger}-\mathbf{A}^{\downarrow^{\dagger}} \mathbf{I}^{\downarrow}\right) \mathbf{A} \mathbf{L}^{-1} \Sigma_{s}^{-2} \mathbf{L}_{k} .
\end{aligned}
$$

Taking the pseudo-inverse of (15) and plugging it into (192), we can show easily that the first term of (186) is equal to zero,

$$
T 1=-\frac{1}{2} \operatorname{Tr}\left(\mathbf{U}_{o} \mathbf{U}_{o}^{H}\right) \sigma_{n}^{2} \mathbf{e}_{k}^{H}\left(\mathbf{A}^{\dagger \dagger} \mathbf{A}^{\dagger}-\mathbf{A}^{\downarrow^{\dagger}} \mathbf{A}^{\downarrow}\right) \mathbf{L}^{-1} \mathbf{\Sigma}_{s}^{-2} \mathbf{L} \mathbf{e}_{k}=0
$$

So far, (186) is reduced to calculating the third term. Substitute $(188),(190),(191),(181)$ and (59) into $T 3$, we have

$$
E\left(\Delta \lambda_{k 2}\right)=\mathbf{e}_{k}^{H}\left(\mathbf{A}^{\downarrow^{H}} \mathbf{A}^{\downarrow}\right)^{-1} \mathbf{L}^{H} \mathbf{\Sigma}_{s}^{-1} \mathbf{V}_{s}^{H} E\left[\mathbf{N}^{H} \mathbf{U}_{o} \mathbf{U}_{o}^{H} \mathbf{I}^{\downarrow^{H}}\right.
$$




$$
\left.\times\left(\mathbf{I}-\mathbf{P}_{\mathbf{A} \downarrow}\right)\left(\mathbf{I}^{\uparrow}-\lambda_{k} \mathbf{I}^{\downarrow}\right) \mathbf{U}_{o} \mathbf{U}_{o}^{H} \mathbf{N}\right] \mathbf{V}, \boldsymbol{\Sigma}_{s}^{-1} \mathbf{L} \mathbf{e}_{k} .
$$

Employing (136), we notice that in (194)

$$
E[\cdot]=\operatorname{Tr}\left[\mathbf{U}_{o} \mathbf{U}_{o}^{H} \mathbf{I}^{\downarrow^{H}}\left(\mathbf{I}-\mathbf{P}_{\mathbf{A} \downarrow}\right)\left(\mathbf{I}^{\dagger}-\lambda_{k} \mathbf{I}^{\downarrow}\right)\right] \mathbf{I} \sigma_{n}^{2}
$$

and further using

$$
\mathbf{U}_{o} \mathbf{U}_{o}^{H}=\mathbf{I}-\mathbf{A}\left(\mathbf{A}^{H} \mathbf{A}\right)^{-1} \mathbf{A}^{H}
$$

(195) becomes

$$
\begin{aligned}
E[\cdot] & =\operatorname{Tr}\left\{\left[\mathbf{I}-\mathbf{A}\left(\mathbf{A}^{H} \mathbf{A}\right)^{-1} \mathbf{A}^{H}\right] \mathbf{I}^{\perp^{H}}\left(\mathbf{I}-\mathbf{P}_{\mathbf{A} \downarrow}\right)\left(\mathbf{I}^{\dagger}-\lambda_{k} \mathbf{I}^{\downarrow}\right)\right\} \mathbf{I} \sigma_{n}^{2} \\
& =\operatorname{Tr}\left\{\mathbf{I}^{\perp^{H}}\left[\mathbf{I}-\mathbf{A}\left(\mathbf{A}^{H} \mathbf{A}\right)^{-1} \mathbf{A}^{H}\right]\left(\mathbf{I}^{\dagger}-\lambda_{k} \mathbf{I}^{\downarrow}\right)\right\} \mathbf{I} \sigma_{n}^{2} \\
& =\left[-\lambda_{k}(L-K-1)-\operatorname{Tr}\left(\mathbf{P}_{\mathbf{A} \downarrow} \mathbf{I}^{\dagger} \mathbf{I}^{\downarrow^{H}}\right)\right] \sigma_{n}^{2} \mathbf{I} .
\end{aligned}
$$

Substitute (197) into (194), we have

$$
\left.E\left(\Delta \lambda_{k 2}\right)\right)=\mathbf{e}_{k}^{H}\left(\mathbf{A}^{\downarrow^{H}} \mathbf{A}^{\downarrow}\right)^{-1} \mathbf{L}^{H} \boldsymbol{\Sigma}_{s}^{-2} \mathbf{L} \mathbf{e}_{k}\left[-\lambda_{k}(L-K-1)-\operatorname{Tr}\left(\mathbf{P}_{\mathbf{A} \downarrow} \mathbf{I}^{\dagger} \mathbf{I}^{\downarrow^{H}}\right)\right] \sigma_{n}^{2}
$$

It is proven in [23]

$$
\mathbf{L}^{H} \mathbf{\Sigma}_{s}^{-2} \mathbf{L} \mathbf{e}_{k}=\mathbf{R}_{s}^{-1} / M
$$

Using (197) and (199), (194) can be further simplified as (128).

\section{D.2 ANGLE PERTURBATION}

The relation between angle and root in a noise environment is

$$
\tilde{\lambda}_{k}=\tilde{c} e^{j \frac{2 \pi d}{\lambda_{c}} \sin \tilde{\theta}_{k}}
$$

First take logarithms of both sides, and substitute $\lambda_{k}+\Delta \lambda_{k}$ for $\tilde{\lambda}_{k}$ and $\theta_{k}+\Delta \theta_{k}$ for $\tilde{\theta}_{k}$. Then expand both sides using Taylor series to the second-order, and equate the imaginary 
parts of both sides as

$$
\Im\left(\ln \lambda_{k}+\frac{\Delta \lambda_{k}}{\lambda_{k}}-\frac{\Delta \lambda_{k}^{2}}{2 \lambda_{k}^{2}}\right)=\frac{2 \pi d}{\lambda_{c}}\left(\sin \theta_{k}+\cos \theta_{k} \Delta \theta_{k}-\frac{\sin \theta_{k}}{2} \Delta \theta_{k}^{2}\right) .
$$

Expanding (200) into the second-order equation as

$$
\Im\left(\ln \lambda_{k}+\frac{\Delta \lambda_{k} 2}{\lambda_{k}}-\frac{\Delta \lambda_{k} 1^{2}}{2 \lambda_{k}^{2}}\right) \stackrel{2}{=} \frac{2 \pi d}{\lambda_{c}}\left(\sin \theta_{k}+\cos \theta_{k} \Delta \theta_{k 2}-\frac{\sin \theta_{k}}{2} \Delta \theta_{k 1}^{2}\right) .
$$

Since $\Im\left(\ln \lambda_{k}\right)=\frac{2 \pi d}{\lambda_{c}} \sin \theta_{k}$, we have

$$
\Im\left(\frac{\Delta \lambda_{k 2}}{\lambda_{k}}-\frac{\Delta \lambda_{k 1}^{2}}{2 \lambda_{k}^{2}}\right) \stackrel{2}{=} \frac{2 \pi d}{\lambda_{c}}\left(\cos \theta_{k} \Delta \theta_{k 2}-\frac{\sin \theta_{k}}{2} \Delta \theta_{k 1}^{2}\right) .
$$

Let $C_{k}=\frac{\lambda_{c}}{2 \pi d \cos \theta_{k}}$,

$$
\Delta \theta_{k 2}=C_{k} \Im\left(\frac{\Delta r_{k 2}}{r_{k}}-\frac{\Delta^{2} r_{k 1}}{2 r_{k}^{2}}\right)+\frac{\tan \theta_{k}}{2} \Delta^{2} \theta_{k 1}
$$

